Systematic Review

\title{
eA Systematic Review and Best Evidence Synthesis of Effectiveness of Therapeutic Facet Joint Interventions in Managing Chronic Spinal Pain
}

\author{
Laxmaiah Manchikanti, MD', Alan D. Kaye, MD, PhD², Mark V. Boswell, MD, PhD², \\ Sanjay Bakshi, MD4, Christopher G. Gharibo, MD5, Vahid Grami, MD ${ }^{6}$, Jay S. Grider, DO, PhD7, \\ Sanjeeva Gupta, MD', Sachin "Sunny" Jha, MD', Dharam P. Mann, MD"10, Devi E. Nampia- \\ parampil, MD ${ }^{11}$, Manohar Lal Sharma, MD ${ }^{12}$, Lindsay N. Shroyer, MD ${ }^{13}$, Vijay Singh, MD ${ }^{14}$, \\ Amol Soin, MD ${ }^{15}$, Ricardo Vallejo, MD ${ }^{16}$, Bradley W. Wargo, DO ${ }^{17}$, and Joshua A. Hirsch, MD ${ }^{18}$
}

From: ${ }^{2}$ Pain Management Center of Paducah, Paducah, KY, and University of Louisville, Louisville, KY; ${ }^{2}$ LSU Health Science Center, New Orleans, LA; ${ }^{3}$ Department of Anesthesiology and Perioperative

Medicine, University of Louisville,

Louisville, KY; ${ }^{4}$ Manhattan Spine and Pain Medicine, New York,

NY; ${ }^{5}$ NYU Langone - Hospital for Joint Diseases, NYU School of Medicine, New York, NY; ${ }^{6}$ Geisinger Medical Center Interventiona Pain Center Woodbine, Danville, PA; ${ }^{7}$ UK Healthcare Pain Services, University of Kentucky, Lexington, $\mathrm{KY} ;{ }^{8}$ Bradford Teaching Hospitals NHS Foundation Trust, Bradford, UK; ${ }^{9}$ Rush University Medical Center (RUMC),Chicago, IL; ${ }^{10} \mathrm{Garden}$ State Pain Management, Whiting NJ; ${ }^{1}$ New York University School of Medicine, New York, NY; ${ }^{12}$ The Walton Centre for Neurology and

Neurosurgery NHS Foundation Trust, Liverpool, UK; ${ }^{13}$ Florida Pain Management Associates, Sebastian, $\mathrm{FL} ;{ }^{14}$ Spine Pain Diagnostics Associates, Niagara, Wl; ${ }^{15}$ Ohio Pain Clinic, Centerville, $\mathrm{OH} ;{ }^{16}$ Millennium Pain Center, Bloomington, IL, Illinois State University, Normal, IL; ${ }^{17}$ The McFarland Clinic, Mary Greeley Medical Center, Department of Pain Medicine, Ames, IA: ${ }^{18}$ Massachusetts General Hospital and Harvard Medical School, Boston, MA.

Address Correspondence: Laxmaiah Manchikanti, M.D. 2831 Lone Oak Road Paducah, Kentucky 42003 E-mail:drlm@thepainmd.com :

Author Affiliation and Disclaimers on P. E573-E574

Manuscript received: 06-14-2015 Accepted for publication: 07-09-2015

Free full manuscript: www.painphysicianjournal.com
Background: The therapeutic spinal facet joint interventions generally used for the treatment of axial spinal pain of facet joint origin are intraarticular facet joint injections, facet joint nerve blocks, and radiofrequency neurotomy. Despite interventional procedures being common as treatment strategies for facet joint pathology, there is a paucity of literature investigating these therapeutic approaches.

Systematic reviews assessing the effectiveness of various therapeutic facet joint interventions have shown there to be variable evidence based on the region and the modality of treatment utilized. Overall, the evidence ranges from limited to moderate.

Objective: To evaluate and update the clinical utility of therapeutic lumbar, cervical, and thoracic facet joint interventions in managing chronic spinal pain.

Study Design: A systematic review of therapeutic lumbar, cervical, and thoracic facet joint interventions for the treatment of chronic spinal pain.

Methods: The available literature on lumbar, cervical, and thoracic facet joint interventions in managing chronic spinal pain was reviewed. The quality assessment criteria utilized were the Cochrane Musculoskeletal Review Group criteria and Interventional Pain Management Techniques - Quality Appraisal of Reliability and Risk of Bias Assessment (IPM - QRB) for randomized trials and Interventional Pain Management Techniques - Quality Appraisal of Reliability and Risk of Bias Assessment for Nonrandomized Studies (IPM - QRBNR) for observational studies.

The level of evidence was classified at 5 levels from Level I to Level V.

Data sources included relevant literature identified through searches on PubMed and EMBASE from 1966 through March 2015, and manual searches of the bibliographies of known primary and review articles.

Outcome Measures: The primary outcome measure was pain relief (short-term relief = up to 6 months and long-term > 6 months). Secondary outcome measures were improvement in functional status, psychological status, return to work, and reduction in opioid intake consumption

Results: A total of 21 randomized controlled trials meeting appropriate inclusion criteria were assessed in this evaluation. A total of 5 observational studies were assessed.

In the lumbar spine, for long-term effectiveness, there is Level II evidence for radiofrequency neurotomy and lumbar facet joint nerve blocks, whereas the evidence is Level III for lumbosacral intraarticular injections.

In the cervical spine, for long-term improvement, there is Level II evidence for cervical radiofrequency neurotomy and cervical facet joint nerve blocks, and Level IV evidence for cervical intraarticular injections.

In the thoracic spine there is Level II evidence for thoracic facet joint nerve blocks and Level IV evidence for radiofrequency neurotomy for long-term improvement. 
Limitations: The limitations of this systematic review include an overall paucity of high quality studies and more specifically the lack of investigations related to thoracic facet joint injections.

Conclusion: Based on the present assessment for the management of spinal facet joint pain, the evidence for long-term improvement is Level II for lumbar and cervical radiofrequency neurotomy, and therapeutic facet joint nerve blocks in the cervical, thoracic, and lumbar spine; Level III for lumbar intraarticular injections; and Level IV for cervical intraarticular injections and thoracic radiofrequency neurotomy.

Key Words: Spinal pain, chronic low back pain, chronic neck pain, chronic thoracic pain, intraarticular facet joint blocks, facet joint nerve blocks, conventional radiofrequency neurotomy, pulsed radiofrequency neurolysis

\section{Pain Physician 2015; 18:E535-E582}

hronic spinal pain with or without extremity pain, chest wall pain, or headaches is commonly encountered in modern day health care, at a time when health care costs and disability are exploding with a corresponding exponential increase in treatment modalities (1-15). Controlled studies have previously established intervertebral discs, facet joints, and sacroiliac joints as potential sources of spinal and extremity pain $(10,16-20)$. It has been described that facet joint degeneration can result from abnormal motion associated with disc degeneration, as well as arthritis, similar to that seen in other synovial joints (21-24). In addition, multiple mechanisms have been postulated as being responsible for modulation of spinal pain related to the facet joints, including capsular stretch, entrapment of synovial villi between the articular surfaces, nerve impingement by osteophytes, and release of inflammatory substances (25-29). The spinal facet joints have been shown to have an abundant nerve supply (30-41); they can cause pain much like what has been reported in normal volunteers who have persistent spinal pain and referred pain into the extremities, chest wall, or head (42-53); are known to be susceptible to arthritic changes, degenerative changes, inflammation, and injury, all of which can lead to a restriction in range of motion and pain upon movement (21-29). By using accepted and proven diagnostic techniques, facet joints have been shown to be a pain generator with subsequent therapeutic application of modalities with significant improvement in pain and disability directed at facet joint innervation $(10,16-19,54)$. Thus, facet joint pain may be diagnosed with reliability based on established controlled diagnostic blocks and may be managed with therapeutic interventions including intraarticular injections, facet joint nerve blocks, or facet joint neurolytic procedures $(10,55-57)$. The diagnostic accuracy and reliability of facet joint nerve blocks for chronic spinal pain have shown that with controlled diagnostic blocks, there is a prevalence of $27 \%$ to $41 \%$ in the low back with a false-positive rate of $25 \%$ to $44 \%$, a prevalence of $36 \%$ to $67 \%$ and a false-positive rate of $27 \%$ to $63 \%$ in the cervical spine, and finally, in the thoracic spine a prevalence rate of $34 \%$ to $48 \%$ with a false-positive rate of $42 \%$ to $48 \%$ (19).

The accurate selection of patients for therapeutic modalities and diagnostic accuracy is crucial, since interventional techniques, specifically facet joint interventions, have shown overall increases of $293 \%$ or $11.1 \%$ per year per 100,000 fee-for-service Medicare population from 2000 to 2013 , compared to $14 \%$ of US population and $64 \%$ of Medicare beneficiaries (13-15). In fact, lumbar facet joint nerve blocks have increased at a rate of $213 \%$, cervical/thoracic facet joint nerve blocks have increased at a rate of $350 \%$, and radiofrequency neurotomy have increased even more at a rate of $522 \%$ for lumbar facet joint radiofrequency neurotomy and $845 \%$ for cervical facet joint radiofrequency neurotomy from 2000 to 2013 in the fee-for-service Medicare population (13-15). Based on the selection criteria of appropriate diagnosis with controlled diagnostic blocks, multiple therapeutic interventions have been assessed in multiple reviews (10,55-61). These systematic reviews demonstrated variable evidence for facet joint neurotomy, facet joint nerve blocks, and intraarticular injections (55-61).

Thus, the debate over the appropriateness of these procedures, continues often with contradictory evidence based on personal and/or professional bias, conservatism, and policy implementations, but not grounded in an appropriate synthesis of the literature (7,58-68). Multiple systematic reviews have been performed based on methodologic assessment but we believe that they display significant bias and have made methodologic errors.

The objective of this systematic review is to assess 
the effectiveness of therapeutic facet joint interventions with appropriate methodology.

\subsection{Methods}

The methodology utilized in this systematic review followed the review process derived from evidencebased systematic reviews and meta-analyses of randomized trials and observational studies (69-73).

\subsection{Criteria for Considering Studies for This Review}

\subsubsection{Types of Studies}

Randomized controlled trials

Observational studies

\subsubsection{Types of Participants}

Patients suffering with chronic neck pain, mid back pain, upper back pain or low back pain of at least 3 months duration.

Patients with acute trauma, fractures, malignancies, and inflammatory diseases were excluded.

\subsubsection{Types of Interventions}

Cervical, thoracic, and lumbar facet joint interventions appropriately performed with proper technique under image guidance (fluoroscopy, computed tomography $[C T]$, or magnetic resonance imaging [MRI]) were included. Blind and ultrasound-guided interventions were excluded.

\subsubsection{Types of Outcome Measures}

- The primary outcome parameter was pain relief.

- The secondary outcome measure was functional status improvement.

\subsection{Literature Search}

All of the available trials in all languages from all countries providing appropriate management with outcome evaluations were considered for inclusion. Searches were performed from the following sources without language restrictions:

1. PubMed from 1966

www.ncbi.nlm.nih.gov/sites/entrez?db=pubmed

2. Cochrane Library www.thecochranelibrary.com/view/0/index.html

3. U.S. National Guideline Clearinghouse (NGC) www.guideline.gov/

4. Previous systematic reviews and cross references
5. Clinical Trials

clinicaltrials.gov/

6. All other sources including non-indexed journals and abstracts

The search period was from 1966 through March 2015.

\subsection{Search Strategy}

The search strategy emphasized chronic spinal pain treated with facet joint interventions. The search terms included: cervical, mid back, and low back pain, facet or zygapophysial joint pain, cervical, thoracic, and lumbar facet injections; cervical, thoracic, and lumbar facet joint nerve blocks; and cervical, thoracic, and lumbar radiofrequency thermoneurolysis.

Search criteria were as follows:

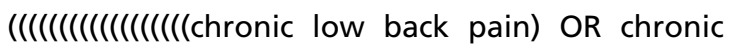
back pain) OR chronic neck pain) OR disc herniation) OR discogenic pain) OR facet joint pain) OR herniated lumbar discs) OR nerve root compression) $O R$ lumbosciatic pain) OR postlaminectomy) OR lumbar surgery syndrome) OR radicular pain) OR radiculitis) OR sciatica) OR spinal fibrosis) OR spinal stenosis) OR zygapophysial)) AND ((((((facet joint) OR zygapophyseal) OR zygapophysial) OR medial branch block) OR diagnostic block) OR radiofrequency) OR intraarticular)

\subsection{Data Collection and Analysis}

The review focused on randomized trials for efficacy. If there were not at least 5 randomized trials, observational studies were utilized. Those patients being studied were those who had been suffering with chronic pain for 3 months or longer. Only facet joint interventions were evaluated. All of the available studies, in all languages, from all countries providing appropriate management and with outcome evaluations of 3 months or longer and statistical evaluations were reviewed. Reports without an appropriate diagnosis, nonsystematic reviews, nonrandomized studies, book chapters, and case reports were excluded.

\subsubsection{Inclusion and Exclusion Criteria}

Randomized trials with at least 3 months of followup and with at least 25 patients in each group or with appropriate sample size determination were included. Observational studies of at least 50 patients with a minimum 6 month follow-up were included when needed (fewer than 5 randomized trials available in any category). 


\subsubsection{Methodological Quality or Validity Assessment}

The quality of each individual article used in this analysis was assessed by Cochrane review criteria (Table 1) (70), Interventional Pain Management techniques Quality Appraisal of Reliability and Risk of Bias Assessment (IPM - QRB) for randomized trials (Table 2) (72), and for observational studies: Interventional Pain Management Techniques - Quality Appraisal of Reliability and Risk of Bias Assessment for Nonrandomized Studies (IPM - QRBNR) (Table 3) (73).

Utilizing Cochrane review criteria, studies meeting the inclusion criteria with a score of at least 8 of 12 were considered high quality and 4 to 7 were considered moderate quality. Those with a score of less than 4 were considered low quality and were excluded.

Based on IPM-QRB criteria for randomized trials, the trials meeting the inclusion criteria that scored less than 16 were considered as low quality and were excluded, those scoring 16 to 31 were considered moderate quality, and those scoring 32 to 48 were considered high quality.

Based on IPM-QRBNR criteria for observational studies, manuscripts meeting the inclusion criteria scoring less than 16 were considered low quality and were excluded, manuscripts scoring 16 to 31 were considered moderate quality, and manuscripts scoring 32 to 48 were considered high quality.

\subsubsection{Data Extraction and Management}

Working independently and in an unblinded, standardized way, 2 review authors established the search criteria, searched for relevant literature, selected the manuscripts, and extracted the data from the included studies. Any disagreement between the 2 reviewers were discussed and debated. If no compromise was reached, another author would review the disagreement and cast the deciding opinion.

Methodological quality assessment was performed by multiple review authors with groups of 2 authors reviewing 4 to 6 manuscripts. The assessment was carried out independently in an unblinded standardized manner to assess the methodological quality and internal validity of all the studies considered for inclusion. The methodological quality assessment was performed in such a way to prevent discrepancies from occurring; if they did occur, a third reviewer was called in and the discrepancy decided by consensus. Continued issues were also discussed with the entire group and resolved.

If there was a conflict of interest with a reviewed manuscript (concerning authorship), if the reviewer was also one of the authors or there was any type of conflict, the involved authors did not review the manuscript for methodological quality assessment.

Table 1. Sources of risk of bias and Cochrane Review rating system.

\begin{tabular}{|l|l|l||}
\hline A & 1. Was the method of randomization adequate? & Yes/No/Unsure \\
\hline B & 2. Was the treatment allocation concealed? & Yes/No/Unsure \\
\hline C & Was knowledge of the allocated interventions adequately prevented during the study? & \\
\hline & 3. Was the patient blinded to the intervention? & Yes/No/Unsure \\
\hline & 4. Was the care provider blinded to the intervention? & Yes/No/Unsure \\
\hline & 5. Was the outcome assessor blinded to the intervention? & Yes/No/Unsure \\
\hline D & Were incomplete outcome data adequately addressed? & Yes/No/Unsure \\
\hline & 6. Was the drop-out rate described and acceptable? & Yes/No/Unsure \\
\hline E & 7. Were all randomized participants analysed in the group to which they were allocated? & Yes/No/Unsure \\
\hline F & Othe reports of the study free of suggestion of selective outcome reporting? & \\
\hline & 9. Were the groups similar at baseline regarding the most important prognostic indicators? & Yes/No/Unsure \\
\hline & 10. Were co-interventions avoided or similar? & Yes/No/Unsure \\
\hline & 11. Was the compliance acceptable in all groups? & Yes/No/Unsure \\
\hline & 12. Was the timing of the outcome assessment similar in all groups? & Yes/No/Unsure \\
\hline
\end{tabular}

Source: Furlan AD, Pennick V, Bombardier C, van Tulder M; Editorial Board, Cochrane Back Review Group. 2009 updated method guidelines for systematic reviews in the Cochrane Back Review Group. Spine (Phila Pa 1976) 2009; 34:1929-1941 (70). 
Table 2. Item checklist for assessment of randomized controlled trials of IPM techniques utilizing IPM-QRB.

\begin{tabular}{|c|c|c|}
\hline \multirow{2}{*}{\begin{tabular}{|l} 
\\
I.
\end{tabular}} & & Scoring \\
\hline & \multicolumn{2}{|l|}{ TRIAL DESIGN AND GUIDANCE REPORTING } \\
\hline \multirow[t]{5}{*}{1.} & CONSORT or SPIRIT & \\
\hline & Trial designed and reported without any guidance & 0 \\
\hline & $\begin{array}{l}\text { Trial designed and reported utilizing minimum criteria other than CONSORT or SPIRIT criteria or trial was } \\
\text { conducted prior to } 2005\end{array}$ & 1 \\
\hline & $\begin{array}{l}\text { Trial implies it was based on CONSORT or SPIRIT without clear description with moderately significant criteria for } \\
\text { randomized trials or the trial was conducted before } 2005\end{array}$ & 2 \\
\hline & $\begin{array}{l}\text { Explicit use of CONSORT or SPIRIT with identification of criteria or trial conducted with high level reporting and } \\
\text { criteria or conducted before } 2005\end{array}$ & 3 \\
\hline II. & \multicolumn{2}{|l|}{ DESIGN FACTORS } \\
\hline \multirow[t]{4}{*}{2.} & Type and Design of Trial & \\
\hline & Poorly designed control group (quasi selection, convenient sampling) & 0 \\
\hline & Proper active-control or sham procedure with injection of active agent & 2 \\
\hline & Proper placebo control (no active solutions into active structures) & 3 \\
\hline \multirow[t]{4}{*}{3.} & Setting/Physician & \\
\hline & General setting with no specialty affiliation and general physician & 0 \\
\hline & Specialty of anesthesia/PMR/neurology/radiology/ortho, etc. & 1 \\
\hline & Interventional pain management with interventional pain management physician & 2 \\
\hline \multirow[t]{5}{*}{4.} & Imaging & \\
\hline & Blind procedures & 0 \\
\hline & Ultrasound & 1 \\
\hline & $\mathrm{CT}$ & 2 \\
\hline & Fluoro & 3 \\
\hline \multirow[t]{5}{*}{5.} & Sample Size & \\
\hline & Less than 50 participants in the study without appropriate sample size determination & 0 \\
\hline & Sample size calculation with less than 25 patients in each group & 1 \\
\hline & Appropriate sample size calculation with at least 25 patients in each group & 2 \\
\hline & Appropriate sample size calculation with 50 patients in each group & 3 \\
\hline \multirow[t]{3}{*}{6.} & Statistical Methodology & \\
\hline & None or inappropriate & 0 \\
\hline & Appropriate & 1 \\
\hline III. & \multicolumn{2}{|l|}{ PATIENT FACTORS } \\
\hline 7. & Inclusiveness of Population & \\
\hline \multirow[t]{4}{*}{7 a. } & For epidural procedures: & \\
\hline & Poorly identified mixed population & 0 \\
\hline & Clearly identified mixed population & 1 \\
\hline & $\begin{array}{l}\text { Disorders specific trials (i.e. well defined spinal stenosis and disc herniation, disorder specific, disc herniation or } \\
\text { spinal stenosis or post surgery syndrome) }\end{array}$ & 2 \\
\hline \multirow[t]{4}{*}{$7 \mathbf{b}$. } & For facet or sacroiliac joint interventions: & \\
\hline & No diagnostic blocks & 0 \\
\hline & Selection with single diagnostic blocks & 1 \\
\hline & Selection with placebo or dual diagnostic blocks & 2 \\
\hline \multirow[t]{4}{*}{8.} & Duration of Pain & \\
\hline & Less than 3 months & 0 \\
\hline & 3 to 6 months & 1 \\
\hline & $>6$ months & 2 \\
\hline
\end{tabular}


Table 2 (cont.). Item checklist for assessment of randomized controlled trials of IPM techniques utilizing IPM-QRB.

\begin{tabular}{|c|c|c|}
\hline & & Scoring \\
\hline \multirow[t]{5}{*}{9.} & Previous Treatments & \\
\hline & Conservative management including drug therapy, exercise therapy, physical therapy, etc. & \\
\hline & Were not utilized & 0 \\
\hline & Were utilized sporadically in some patients & 1 \\
\hline & Were utilized in all patients & 2 \\
\hline \multirow[t]{5}{*}{10.} & Duration of Follow-up with Appropriate Interventions & \\
\hline & $\begin{array}{l}\text { Less than } 3 \text { months or } 12 \text { weeks for epidural or facet joint procedures, etc., and } 6 \text { months for intradiscal procedures } \\
\text { and implantables }\end{array}$ & 0 \\
\hline & 3 to 6 months for epidural or facet joint procedures, etc., or 1 year for intradiscal procedures or implantables & 1 \\
\hline & $\begin{array}{l}6 \text { months to } 17 \text { months for epidurals or facet joint procedures, etc., and } 2 \text { years or longer for discal procedures and } \\
\text { implantables }\end{array}$ & 2 \\
\hline & $\begin{array}{l}18 \text { months or longer for epidurals and facet joint procedures, etc., or } 5 \text { years or longer for discal procedures and } \\
\text { implantables }\end{array}$ & 3 \\
\hline IV. & OUTCOMES & \\
\hline \multirow[t]{6}{*}{11.} & Outcomes Assessment Criteria for Significant Improvement & \\
\hline & $\begin{array}{l}\text { No descriptions of outcomes } \\
\text { OR } \\
<20 \% \text { change in pain rating or functional status }\end{array}$ & 0 \\
\hline & $\begin{array}{l}\text { Pain rating with a decrease of } 2 \text { or more points or more than } 20 \% \text { reduction } \\
\text { OR } \\
\text { functional status improvement of more than } 20 \%\end{array}$ & 1 \\
\hline & $\begin{array}{l}\text { Pain rating with decrease of } \geq 2 \text { points } \\
\text { AND } \\
\geq 20 \% \text { change or functional status improvement of } \geq 20 \%\end{array}$ & 2 \\
\hline & $\begin{array}{l}\text { Pain rating with a decrease of } 3 \text { or more points or more than } 50 \% \text { reduction } \\
\text { OR } \\
\text { functional status improvement with a } 50 \% \text { or } 40 \% \text { reduction in disability score }\end{array}$ & 2 \\
\hline & Significant improvement with pain and function $\geq 50 \%$ or 3 points and $40 \%$ reduction in disability scores & 4 \\
\hline \multirow[t]{4}{*}{12.} & Analysis of All Randomized Participants in the Groups & \\
\hline & Not performed & 0 \\
\hline & Performed without intent-to-treat analysis without inclusion of all randomized participants & 1 \\
\hline & All participants included with or without intent-to-treat analysis & 2 \\
\hline \multirow[t]{4}{*}{13.} & Description of Drop Out Rate & \\
\hline & No description of dropouts, despite reporting of incomplete data or $\geq 20 \%$ withdrawal & 0 \\
\hline & Less than $20 \%$ withdrawal in one year in any group & 1 \\
\hline & Less than $30 \%$ withdrawal at 2 years in any group & 2 \\
\hline \multirow[t]{4}{*}{14.} & Similarity of Groups at Baseline for Important Prognostic Indicators & \\
\hline & Groups dissimilar with significant influence on outcomes with or without appropriate randomization and allocation & 0 \\
\hline & Groups dissimilar without influence on outcomes despite appropriate randomization and allocation & 1 \\
\hline & Groups similar with appropriate randomization and allocation & 2 \\
\hline \multirow[t]{3}{*}{15.} & Role of Co-Interventions & \\
\hline & Co-interventions were provided but were not similar in the majority of participants & 0 \\
\hline & No co-interventions or similar co-interventions were provided in the majority of the participants & 1 \\
\hline V. & RANDOMIZATION & \\
\hline \multirow[t]{3}{*}{16.} & Method of Randomization & \\
\hline & Quasi randomized or poorly randomized or not described & 0 \\
\hline & Adequate randomization (coin toss, drawing of balls of different colors, drawing of ballots) & 1 \\
\hline
\end{tabular}


Table 2 (cont.). Item checklist for assessment of randomized controlled trials of IPM techniques utilizing IPM - QRB.

\begin{tabular}{|c|c|c|}
\hline & $\begin{array}{l}\text { High quality randomization (computer generated random sequence, pre-ordered sealed envelopes, sequentially } \\
\text { ordered vials, telephone call, pre-ordered list of treatment assignments, etc.) }\end{array}$ & 2 \\
\hline VI. & ALLOCATION CONCEALMENT & \\
\hline \multirow[t]{4}{*}{17.} & Concealed Treatment Allocation & \\
\hline & Poor concealment of allocation (open enrollment) or inadequate description of concealment & 0 \\
\hline & $\begin{array}{l}\text { Concealment of allocation with borderline or good description of the process with probability of failure of } \\
\text { concealment }\end{array}$ & 1 \\
\hline & $\begin{array}{l}\text { High quality concealment with strict controls (independent assignment without influence on the assignment } \\
\text { sequence) }\end{array}$ & 2 \\
\hline VII. & BLINDING & \\
\hline \multirow[t]{3}{*}{18.} & Patient Blinding & \\
\hline & Patients not blinded & 0 \\
\hline & Patients blinded adequately & 1 \\
\hline \multirow[t]{3}{*}{19.} & Care Provider Blinding & \\
\hline & Care provider not blinded & 0 \\
\hline & Care provider blinded adequately & 1 \\
\hline \multirow[t]{3}{*}{20.} & Outcome Assessor Blinding & \\
\hline & Outcome assessor not blinded or was able to identify the groups & 0 \\
\hline & $\begin{array}{l}\text { Performed by a blinded independent assessor with inability to identify the assignment-based provider intervention } \\
\text { (i.e., subcutaneous injection, intramuscular distant injection, difference in preparation or equipment use, numbness } \\
\text { and weakness, etc.) }\end{array}$ & 1 \\
\hline VIII. & CONFLICTS OF INTEREST & \\
\hline \multirow[t]{7}{*}{21.} & Funding and Sponsorship & \\
\hline & Trial included industry employees & -3 \\
\hline & $\begin{array}{l}\text { Industry employees involved; high levels of funding with remunerations by industry or an organization funded with } \\
\text { conflicts }\end{array}$ & -3 \\
\hline & Industry or organizational funding with reimbursement of expenses with some involvement & 0 \\
\hline & Industry or organization funding of expenses without involvement & 1 \\
\hline & Funding by internal resources only with supporting entity unrelated to industry & 2 \\
\hline & Governmental funding without conflict such as NIH, NHS, AHRQ & 3 \\
\hline \multirow[t]{8}{*}{22.} & Conflicts of Interest & \\
\hline & None disclosed with potential implied conflict & 0 \\
\hline & Marginally disclosed with potential conflict & 1 \\
\hline & Well disclosed with minor conflicts & 2 \\
\hline & Well disclosed with no conflicts & 3 \\
\hline & Hidden conflicts with poor disclosure & -1 \\
\hline & Misleading disclosure with conflicts & -2 \\
\hline & Major impact related to conflicts & -3 \\
\hline & TOTAL MAXIMUM & 48 \\
\hline
\end{tabular}

Source: Manchikanti L, Hirsch JA, Cohen SP, Heavner JE, Falco FJE, Diwan S, Boswell MV, Candido KD, Onyewu O, Zhu J, Sehgal N, Kaye AD, Benyamin RM, Helm II S, Singh V, Datta S, Abdi S, Christo PJ, Hameed H, Hameed M, Vallejo R, Pampati V, Racz GB, Raj PP. Assessment of methodologic quality of randomized trials of interventional techniques: Development of interventional pain management specific instrument. Pain Physician 2014; 17:E263-E290 (72). 
Table 3. IPM checklist for assessment of nonrandomized or observational studies of IPM techniques utilizing IPM-QRBNR.

\begin{tabular}{|c|c|c|}
\hline I. & STUDY DESIGN AND GUIDANCE REPORTING & Scoring \\
\hline \multirow[t]{6}{*}{1.} & STROBE or TREND Guidance & \\
\hline & Case Report/Case Series & 0 \\
\hline & Study designed without any guidance & 1 \\
\hline & Study designed with minimal criteria and reporting with or without guidance & 2 \\
\hline & $\begin{array}{l}\text { Study designed with moderately significant criteria or implies it was based on STROBE or TREND without clear } \\
\text { description or the study was conducted before } 2011 \text { or similar criteria utilized with study conducted before } 2011\end{array}$ & 3 \\
\hline & $\begin{array}{l}\text { Designed with high level criteria or explicitly uses STROBE or TREND with identification of criteria or conducted prior } \\
\text { to } 2011\end{array}$ & 4 \\
\hline II. & DESIGN FACTORS & \\
\hline \multirow[t]{6}{*}{2.} & Study Design and Type & \\
\hline & Case report or series (uncontrolled - longitudinal) & 0 \\
\hline & Retrospective cohort or cross-sectional study & 1 \\
\hline & Prospective cohort case-control study & 2 \\
\hline & Prospective case control study & 3 \\
\hline & Prospective, controlled, nonrandomized & 4 \\
\hline \multirow[t]{4}{*}{3.} & Setting/Physician & \\
\hline & General setting with no specialty affiliation and general physician & 0 \\
\hline & Specialty of anesthesia/PMR/neurology, etc. & 1 \\
\hline & Interventional pain management with interventional pain management physician & 2 \\
\hline \multirow[t]{5}{*}{4.} & Imaging & \\
\hline & Blind procedures & 0 \\
\hline & Ultrasound & 1 \\
\hline & $\mathrm{CT}$ & 2 \\
\hline & Fluoro & 3 \\
\hline \multirow[t]{6}{*}{5.} & Sample Size & \\
\hline & Less than 100 participants without appropriate sample size determination & 0 \\
\hline & At least 100 participants in the study without appropriate sample size determination & 1 \\
\hline & Sample size calculation with less than 50 patients in each group & 2 \\
\hline & Appropriate sample size calculation with at least 50 patients in each group & 3 \\
\hline & Appropriate sample size calculation with 100 patients in each group & 4 \\
\hline \multirow[t]{4}{*}{6.} & Statistical Methodology & \\
\hline & None & 0 \\
\hline & Some statistics & 1 \\
\hline & Appropriate & 2 \\
\hline III. & PATIENT FACTORS & \\
\hline 7. & Inclusiveness of Population & \\
\hline \multirow[t]{5}{*}{$7 \mathrm{a}}$. & For epidural procedures: & \\
\hline & Poorly identified mixed population & 1 \\
\hline & Poorly identified mixed population with large sample $(\geq 200)$ & 2 \\
\hline & Clearly identified mixed population & 3 \\
\hline & $\begin{array}{l}\text { Disorders specific trials (i.e. well defined spinal stenosis and disc herniation, disorder specific, disc herniation or spinal } \\
\text { stenosis or post surgery syndrome) }\end{array}$ & 4 \\
\hline \multirow[t]{3}{*}{$7 \mathrm{~b}}$. & For facet or sacroiliac joint interventions: & \\
\hline & No specific selection criteria & 1 \\
\hline & No diagnostic blocks based on clinical symptomatology & 2 \\
\hline
\end{tabular}


Table 3 (cont.) . IPM checklist for assessment of nonrandomized or observational studies of IPM techniques utilizing IPM-QRBNR.

\begin{tabular}{|c|c|c|}
\hline & Selection with single diagnostic blocks & 3 \\
\hline & Selection with placebo or dual diagnostic blocks & 4 \\
\hline \multirow[t]{4}{*}{8.} & Duration of Pain & \\
\hline & Less than 3 months & 0 \\
\hline & 3 to 6 months & 1 \\
\hline & $>6$ months & 2 \\
\hline \multirow[t]{5}{*}{9.} & Previous Treatments & \\
\hline & Conservative management including drug therapy, exercise therapy, physical therapy, etc. & \\
\hline & Were not utilized & 0 \\
\hline & Were utilized sporadically in some patients & 1 \\
\hline & Were utilized in all patients & 2 \\
\hline \multirow[t]{5}{*}{10.} & Duration of Follow-up with Appropriate Interventions & \\
\hline & $\begin{array}{l}\text { Less than } 3 \text { months or less for epidural or facet joint procedures, etc., and } 6 \text { months for intradiscal procedures and } \\
\text { implantables }\end{array}$ & 1 \\
\hline & 3-6 months for epidural or facet joint procedures, etc., or one year for intradiscal procedures or implantables & 2 \\
\hline & 6-12 months for epidurals or facet joint procedures, etc., and 2 years or longer for discal procedures and implantables & 3 \\
\hline & $\begin{array}{l}18 \text { months or longer for epidurals and facet joint procedures, etc., or } 5 \text { years or longer for discal procedures and } \\
\text { implantables }\end{array}$ & 4 \\
\hline IV. & OUTCOMES & \\
\hline \multirow[t]{6}{*}{11.} & Outcomes Assessment Criteria for Significant Improvement & \\
\hline & $\begin{array}{l}\text { No descriptions of outcomes } \\
\text { OR } \\
<20 \% \text { change in pain rating or functional status }\end{array}$ & 0 \\
\hline & $\begin{array}{l}\text { Pain rating with a decrease of } 2 \text { or more points or more than } 20 \% \text { reduction } \\
\text { OR } \\
\text { functional status improvement of more than } 20 \%\end{array}$ & 1 \\
\hline & $\begin{array}{l}\text { Pain rating with decrease of } \geq 2 \text { points } \\
\text { AND } \\
\geq 20 \% \text { change or functional status improvement of } \geq 20 \%\end{array}$ & 2 \\
\hline & $\begin{array}{l}\text { Pain rating with a decrease of } 3 \text { or more points or more than } 50 \% \text { reduction } \\
\text { OR } \\
\text { functional status improvement with a } 50 \% \text { or } 40 \% \text { reduction in disability score }\end{array}$ & 2 \\
\hline & Significant improvement with pain and function $\geq 50 \%$ or 3 points and $40 \%$ reduction in disability scores & 4 \\
\hline \multirow[t]{4}{*}{12.} & Description of Drop Out Rate & \\
\hline & No description despite reporting of incomplete data or more than $30 \%$ withdrawal & 0 \\
\hline & Less than $30 \%$ withdrawal in one year in any group & 1 \\
\hline & Less than $40 \%$ withdrawal at 2 years in any group & 2 \\
\hline \multirow[t]{4}{*}{13.} & Similarity of Groups at Baseline for Important Prognostic Indicators & \\
\hline & No groups or groups dissimilar with significant influence on outcomes & 0 \\
\hline & Groups dissimilar without significant influence on outcomes & 1 \\
\hline & Groups similar & 2 \\
\hline \multirow[t]{3}{*}{14.} & Role of Co-Interventions & \\
\hline & Dissimilar co-interventions or similar co-interventions in some of the participants & 1 \\
\hline & No co-interventions or similar co-interventions in majority of the participants & 2 \\
\hline V. & ASSIGNMENT & \\
\hline \multirow[t]{3}{*}{15.} & Method of Assignment of Participants & \\
\hline & Case report/case series or selective assignment based on outcomes or retrospective evaluation based on clinical criteria & 1 \\
\hline & Prospective study with inclusion without specific criteria & 2 \\
\hline
\end{tabular}


Table 3 (cont.) . IPM checklist for assessment of nonrandomized or observational studies of IPM techniques utilizing IPM-QRBNR.

\begin{tabular}{|l|l|c||}
\hline & Retrospective method with inclusion of all participants or random selection of retrospective data & 3 \\
\hline & $\begin{array}{l}\text { Prospective, well-defined assignment of methodology and inclusion criteria (quasi randomization, matching, } \\
\text { stratification, etc.) }\end{array}$ & 4 \\
\hline VI. & CONFLICTS OF INTEREST & \\
\hline 16. & Funding and Sponsorship & -3 \\
\hline & Trial included industry employees with or without proper disclosure & -3 \\
\hline & $\begin{array}{l}\text { Industry employees involved; high levels of funding with remunerations by industry or an organization funded with } \\
\text { conflicts }\end{array}$ & Industry or organizational funding with reimbursement of expenses with some involvement or no information available \\
\hline & Industry or organization funding of expenses without involvement & 0 \\
\hline & Funding by internal resources only & 1 \\
\hline & Governmental funding without conflict such as NIH, NHS, AHRQ & 2 \\
\hline
\end{tabular}

Source: Manchikanti L, Hirsch JA, Heavner JE, Cohen SP, Benyamin RM, Sehgal N, Falco FJE, Vallejo R, Onyewu O, Zhu J, Kaye AD, Boswell MV, Helm II S, Candido KD, Diwan S, Simopoulos TT, Singh V, Pampati V, Racz GB, Raj PP. Development of an interventional pain management specific instrument for methodologic quality assessment of nonrandomized studies of interventional techniques. Pain Physician 2014; 17:E291E317 (73).

\subsubsection{Measurement of Treatment Effect in Data Synthesis (Meta-Analysis)}

If the literature search provided at least 3 randomized trials meeting the inclusion criteria and they are clinically homogenous for each modality and region evaluated, a meta-analysis was performed.

Data were summarized using a meta-analysis when at least 3 trials per type of modality were available that met the inclusion criteria (e.g., intraarticular injections, facet joint nerve blocks, and radiofrequency thermoneurolysis) of clinical and statistical homogeneity.

Qualitative (the direction of a treatment effect) and quantitative (the magnitude of a treatment effect) conclusions were evaluated. A random-effects meta-analysis to pool data was also used. For placebo-controlled trials, the net effect between 2 treatments was utilized. However, for active-controlled trials, the differences between baseline and follow-up period were utilized.

\subsection{Outcome of the Studies}

According to reports from trials that studied general chronic pain, a clinically meaningful pain score change is considered to be, at a minimum, a 2-point change on a 0 to 10 scale (or 20 percentage points) (74), chronic musculoskeletal pain (75), and chronic low back pain $(71-73,75,76)$, which have been commonly utilized. Traditional criteria of minimum or meaningful improvements have been criticized as clinically irrelevant (71-73,77-81). Thus, recent descriptions of clinically meaningful improvement considered more robust outcomes with either pain relief and functional status improvement of $50 \%$ (82-97). The following outcomes were considered clinically meaningful or significant: a 3-point or greater change on an 11-point pain scale (0$10)$, or a $50 \%$ pain improvement from baseline and a $40 \%$ or greater improvement in functional status.

A trial was judged to be positive if the facet joint intervention was clinically relevant and effective, either with a placebo control or active control. This indicates that the difference in the effect for the primary outcome measure was statistically significant on the conventional $5 \%$ level. Negative studies were those where the study treatments showed no difference or there was no improvement from baseline. Outcomes were reported at one, 3, 6, and 12 months. For observational studies, appropriate outcomes were reported with positive or negative results at 3 months, 6 months, and one-year or longer with effectiveness demonstrated when a study was judged to be positive. If a lack of effectiveness was identified in the study, it was judged to be negative.

\subsection{Summary Measures}

Summary measures included a $50 \%$ or more reduction of pain in at least $50 \%$ of the patients, or at least a 3-point decrease in pain scores and a relative risk of adverse events, including side effects.

\subsection{Analysis of Evidence}

The analysis of the evidence was performed based on best evidence synthesis developed from modifica- 
Table 4. Qualitative modified approach to grading of evidence.

\begin{tabular}{|l|l||}
\hline Level I & Evidence obtained from multiple relevant high quality randomized controlled trials \\
\hline Level II & $\begin{array}{l}\text { Evidence obtained from at least one relevant high quality randomized controlled trial or multiple relevant moderate or low } \\
\text { quality randomized controlled trials }\end{array}$ \\
\hline Level III & $\begin{array}{l}\text { Evidence obtained from at least one relevant moderate or low quality randomized controlled trial with multiple relevant } \\
\text { observational studies } \\
\text { or } \\
\text { Evidence obtained from at least one relevant high quality nonrandomized trial or observational study with multiple moderate } \\
\text { or low quality observational studies }\end{array}$ \\
\hline Level IV & Evidence obtained from multiple moderate or low quality relevant observational studies \\
\hline Level V & Opinion or consensus of large group of clinicians and/or scientists \\
\hline \hline
\end{tabular}

Source: Manchikanti L, Falco FJE, Benyamin RM, Kaye AD, Boswell MV, Hirsch JA. A modified approach to grading of evidence. Pain Physician 2014; 17:E319-E325 (105).

tion of multiple available criteria including those of the United States Preventive Services Task Force (USPSTF) and Cochrane review criteria as illustrated in Table 4 (98-104).

The analysis was conducted utilizing best evidence synthesis using 5 levels of evidence ranging from strong to opinion- or consensus-based (105).

At least 2 of the review authors independently, in an unblinded standardized manner, analyzed the evidence. Any disagreements between reviewers were resolved by a third author and consensus. If there were any conflicts of interest (e.g., authorship), those reviewers were recused from assessment and analysis for the study in question.

\subsection{Results}

Figure 1 shows a flow diagram of the study selection of therapeutic intervention trials and studies.

Based on comprehensive search criteria there were multiple studies considered for inclusion (82-84, 106-164).

Multiple randomized trials, duplicates, and all nonrandomized trials were excluded. Table 5 is a partial list of excluded trials that did not meet inclusion criteria. Subsequently, 21 randomized trials $(82-84,108,110,112-$ $124,128,129)$ and 5 observational studies were included (152,159-162).

There were 3 trials $(82,112,117)$ that evaluated therapeutic lumbar facet joint nerve blocks, 9 trials (108,113$118,122,123)$ that evaluated lumbar facet joint radiofrequency neurolysis, and 5 trials $(110,111,121,123,124)$ that evaluated lumbar intraarticular injections that met the inclusion criteria. Even though there were only 3 randomized controlled trials, there were no observational therapeutic facet joint nerve block studies available for inclusion.
There was one trial (128) that evaluated the efficacy of cervical facet joint nerve radiofrequency thermoneurolysis, one trial (83) that evaluated the efficacy of cervical facet joint nerve blocks, and 2 trials $(119,129)$ that evaluated cervical intraarticular injections that met inclusion criteria. Thus, 3 observational studies assessing radiofrequency neurotomy of the cervical spine (159161) were included in the assessment of radiofrequency neurotomy. In addition, one prospective evaluation of cervical facet joint nerve blocks was also included (152).

There was one trial (84) that evaluated therapeutic thoracic facet joint nerve blocks and one trial (120) that evaluated thoracic facet joint radiofrequency neurolysis that met the inclusion criteria. Thus, one study of thoracic facet joint nerve blocks (162) was included. There were no other studies meeting inclusion criteria.

\subsection{Methodological Quality Assessment}

A methodological quality assessment of the randomized controlled trials meeting inclusion criteria was carried out utilizing Cochrane review criteria and IPMQRB criteria for randomized trials as shown in Tables 6 and 7 and IPM - QRBNR for nonrandomized studies as shown in Table 8.

Utilizing Cochrane review criteria, studies meeting the inclusion criteria with at least a score of 8 of 12 were considered high quality and a score of 4 to 7 were considered moderate quality. A score lower than 4 was considered low quality and those studies were excluded.

Based on IPM-QRB criteria for randomized trials and IPM-QRBNR for observational studies, the trials meeting the inclusion criteria with scores of less than 16 were considered low quality and were excluded, manuscripts with scores of 16 to 31 were considered moderate quality, and scores of 32 to 48 or higher were considered as high quality trials. 


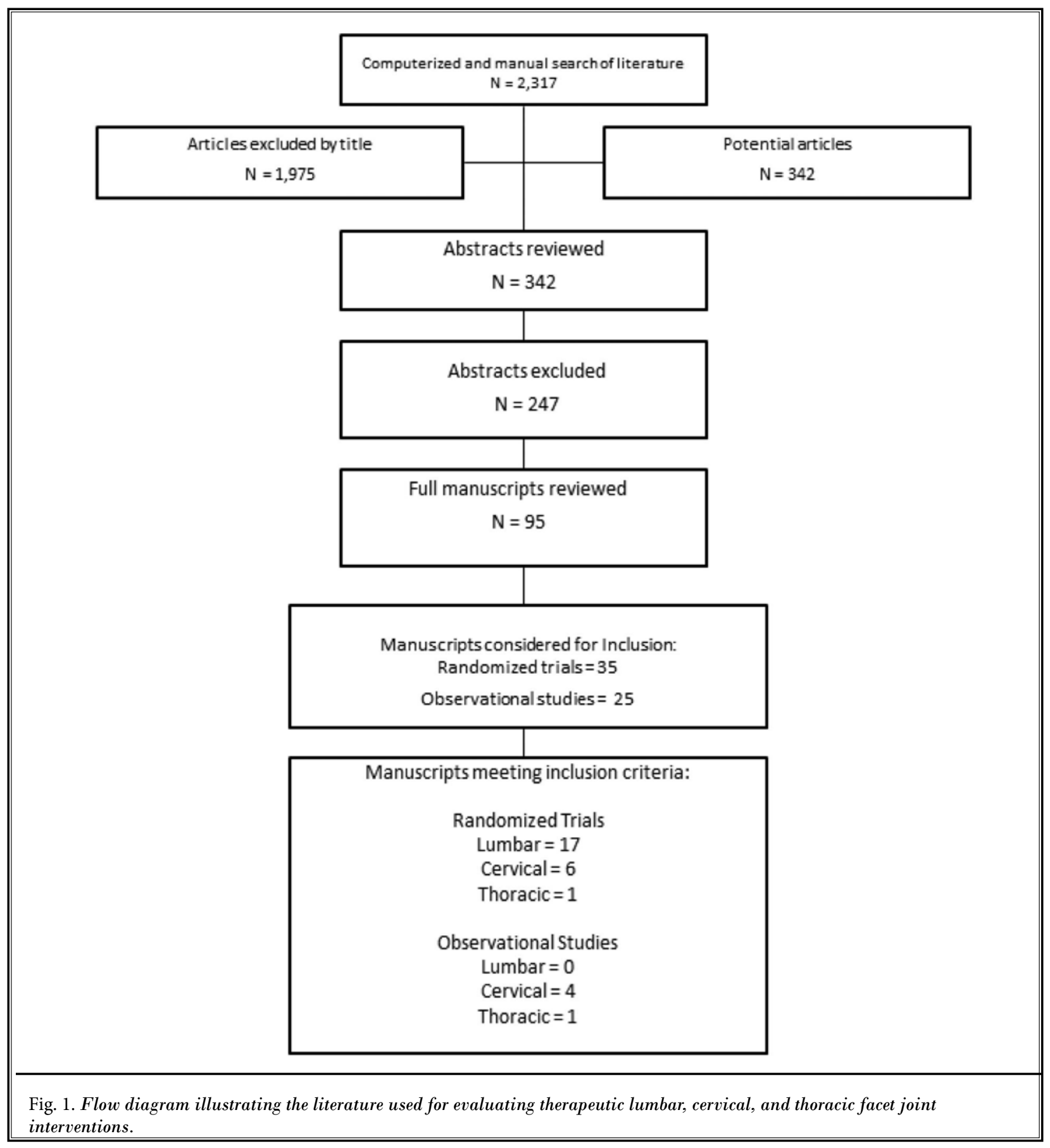

\subsection{Meta-Analysis}

If there were more than 2 trials meeting clinical homogeneity criteria, they were further assessed for homogeneity, and a meta-analysis was performed. There were 9 trials assessing lumbar radiofrequency neurotomy $(108,113-118,122,123), 5$ trials assessing lumbar

intraarticular injection therapy $(110,111,121,123,124)$, and 3 trials assessing lumbar facet joint nerve blocks $(82,112,117)$. However, all modalities in the cervical spine and the thoracic spine had 2 or fewer. An assessment of clinical and methodological homogeneity 
Table 5. List of excluded randomized trials with brief explanation.

\begin{tabular}{|c|c|c|c|c|}
\hline \multirow[b]{2}{*}{ Study } & \multirow{2}{*}{$\begin{array}{l}\text { Condition } \\
\text { Studied }\end{array}$} & \multirow{2}{*}{$\begin{array}{l}\text { Number of } \\
\text { Patients }\end{array}$} & \multicolumn{2}{|r|}{ Reason for Exclusion } \\
\hline & & & $\begin{array}{l}\text { Followup } \\
\text { Period }\end{array}$ & Other Reason(s) \\
\hline \multicolumn{5}{|c|}{ LUMBAR FACET JOINT INTERVENTIONS } \\
\hline $\begin{array}{l}\text { Lilius et al (131) } \\
\text { Randomized }\end{array}$ & $\begin{array}{l}\text { Chronic low back } \\
\text { pain }\end{array}$ & 109 & 3 months & $\begin{array}{l}\text { Study with short-term follow-up along with lack of diagnostic } \\
\text { blocks and comparison of intraarticular or extraarticular } \\
\text { injections with a large volume of injection. At best, this study may } \\
\text { be appropriate for a diagnostic study with a single block. }\end{array}$ \\
\hline $\begin{array}{l}\text { Marks et al (132) } \\
\text { Randomized }\end{array}$ & $\begin{array}{l}\text { Chronic low back } \\
\text { pain }\end{array}$ & 86 & 3 months & $\begin{array}{l}\text { The authors compared facet joint nerve blocks and intraarticular } \\
\text { injections with high volume injections with very short-term } \\
\text { follow-up in a randomized trial as diagnostic blocks. }\end{array}$ \\
\hline $\begin{array}{l}\text { Nash (133) } \\
\text { Randomized }\end{array}$ & $\begin{array}{l}\text { Chronic low back } \\
\text { pain }\end{array}$ & 67 & 3 months & $\begin{array}{l}\text { The authors compared the effectiveness of intraarticular } \\
\text { injections with medial branch blocks on a short-term basis with } \\
\text { no controlled local anesthetic blocks, and with lack of long-term } \\
\text { follow-up and outcomes }\end{array}$ \\
\hline $\begin{array}{l}\text { Leclaire et al (134) } \\
\text { Randomized, } \\
\text { Placebo-Controlled }\end{array}$ & $\begin{array}{l}\text { Chronic low back } \\
\text { pain }\end{array}$ & 70 & 12 weeks & $\begin{array}{l}\text { Relatively small study; however, technique and the diagnostic } \\
\text { evaluation with intraarticular injections were inappropriate. The } \\
\text { authors have admitted that the results might not be applicable in } \\
\text { clinical practice. }\end{array}$ \\
\hline $\begin{array}{l}\text { Gallagher et al (135) } \\
\text { Randomized }\end{array}$ & $\begin{array}{l}\text { Chronic low back } \\
\text { pain }\end{array}$ & 41 & $\begin{array}{l}\text { One month } \\
\text { and } 6 \text { months }\end{array}$ & $\begin{array}{l}\text { Authors evaluated } 60 \text { patients with a single block and randomized } \\
\text { them into } 2 \text { groups with } 41 \text { patients testing positive. The study } \\
\text { showed improvement at one month and } 6 \text { months; however, the } \\
\text { inclusion criteria, the technical considerations, and statistical } \\
\text { analysis were considered as flawed. }\end{array}$ \\
\hline $\begin{array}{l}\text { Kroll et al (136) } \\
\text { Randomized }\end{array}$ & $\begin{array}{l}\text { Acute low back } \\
\text { pain }\end{array}$ & 50 & 3 months & $\begin{array}{l}\text { Conventional and pulsed radiofrequency neurotomy were studied } \\
\text { in acute low back pain. }\end{array}$ \\
\hline $\begin{array}{l}\text { Kader et al (140) } \\
\text { Randomized }\end{array}$ & $\begin{array}{l}\text { Chronic } \\
\text { nonspecific low } \\
\text { back pain with or } \\
\text { without leg pain }\end{array}$ & 63 & 10 weeks & $\begin{array}{l}\text { Patients were randomized into } 3 \text { groups with back education and } \\
\text { standard physiotherapy for } 10 \text { weeks, back education and gym ball } \\
\text { exercise for } 10 \text { weeks, or perifacet injection into the lumbar multifidus } \\
\text { muscle with methylprednisolone. Since there was no facet joint } \\
\text { injection, the study failed to meet the criteria for inclusion. }\end{array}$ \\
\hline $\begin{array}{l}\text { Wen et al (126) } \\
\text { Randomized }\end{array}$ & Low back pain & 20 & 6 weeks & $\begin{array}{l}\text { Twenty with } 10 \text { patients in each group receiving facet joint blocks } \\
\text { either blindly or guided by ultrasound; however, the needle tip was } \\
\text { confirmed by CT in both groups. Small sample size with short- } \\
\text { term follow-up of } 6 \text { weeks utilizing either a blind technique or } \\
\text { ultrasound-guided lumbar facet joint injections. }\end{array}$ \\
\hline \multicolumn{5}{|c|}{ CERVICAL FACET JOINT INTERVENTIONS } \\
\hline $\begin{array}{l}\text { Obernauer et al } \\
\text { (125) } \\
\text { Randomized }\end{array}$ & $\begin{array}{l}\text { Subacute chronic } \\
\text { facet-joint- } \\
\text { associated neck pain } \\
\text { of the middle or } \\
\text { lower cervical spine }\end{array}$ & 40 & 4 weeks & $\begin{array}{l}\text { Injection of local anesthetic and steroids into cervical facet joints } \\
\text { either under CT scanning or ultrasonography. The short-term } \\
\text { follow-up in patients with subacute pain was done without } \\
\text { diagnostic blocks. }\end{array}$ \\
\hline $\begin{array}{l}\text { Slappendel et al } \\
\text { (148) } \\
\text { Randomized }\end{array}$ & Cervicobrachialgia & 61 & 3 months & $\begin{array}{l}\text { The authors evaluated the efficacy of radiofrequency lesioning of } \\
\text { the cervical dorsal root ganglion. }\end{array}$ \\
\hline $\begin{array}{l}\text { Haspeslagh et al } \\
\text { (149) } \\
\text { Randomized }\end{array}$ & $\begin{array}{l}\text { Cervicogenic } \\
\text { headache }\end{array}$ & 30 & 48 weeks & $\begin{array}{l}\text { In this study, } 30 \text { patients with cervicogenic headache were } \\
\text { evaluated. This study was problematic, not only in the diagnosis } \\
\text { but also in the application of technique. } \\
\text { The authors claim that they developed a sequence of various } \\
\text { cervical radiofrequency neurotomies that proved successful in a } \\
\text { prospective pilot trial with } 15 \text { chronic headache patients. Their } \\
\text { diagnosis was not established by controlled diagnostic blocks; and } \\
\text { the treatments targeted toward different structures: cervical facet } \\
\text { joints and dorsal root ganglia compared to occipital nerves. }\end{array}$ \\
\hline
\end{tabular}




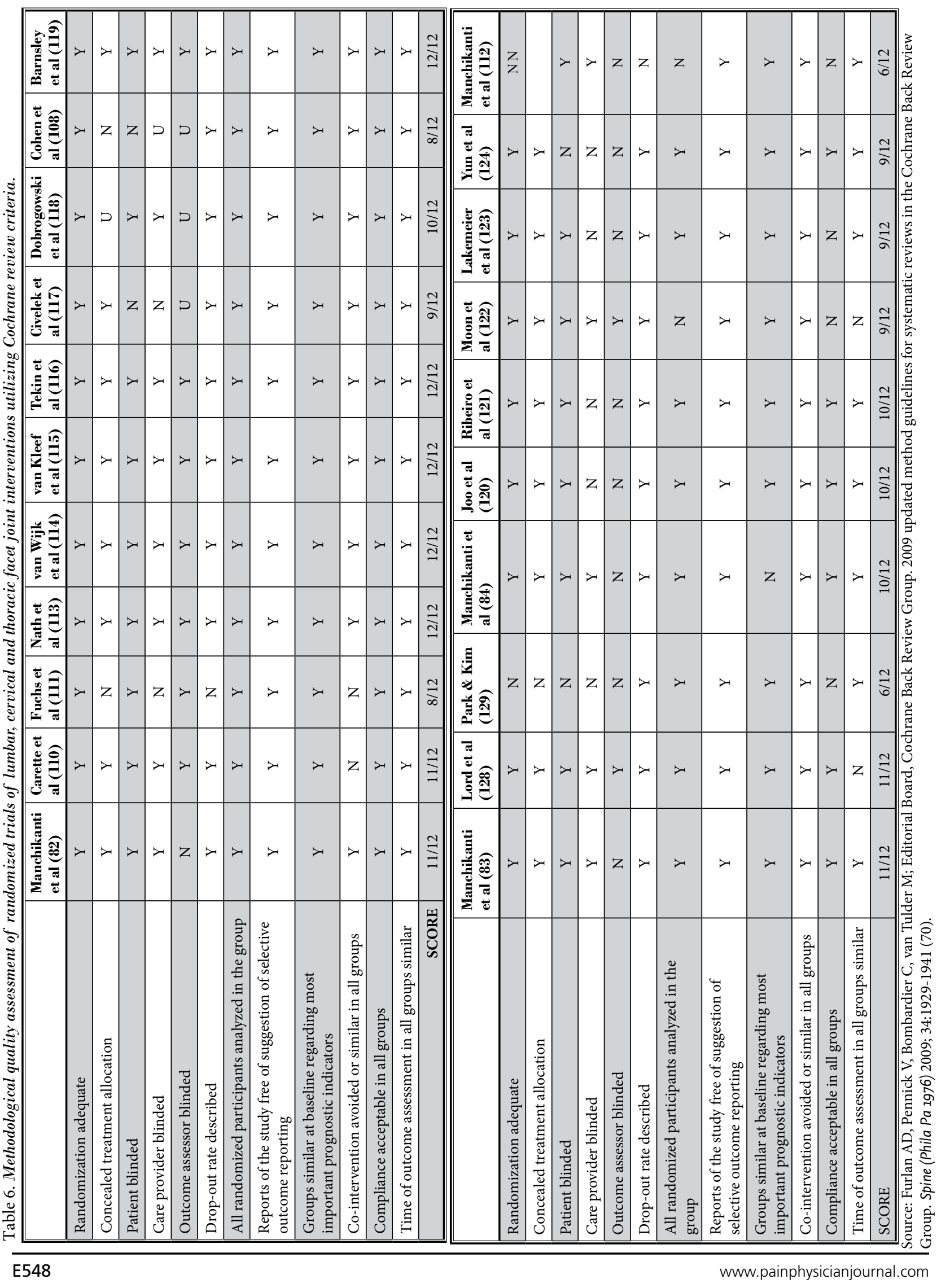


Effectiveness of Therapeutic Facet Joint Interventions

Table 7. Methodologic quality assessment of randomized trials of lumbar, cervical and thoracic facet joint interventions utilizing IPM -QRB criteria.

\begin{tabular}{|c|c|c|c|c|c|c|c|c|c|c|c|}
\hline & & $\begin{array}{l}\text { Manchikanti } \\
\text { et al (82) }\end{array}$ & $\begin{array}{l}\text { Carette } \\
\text { et al } \\
(110)\end{array}$ & $\begin{array}{l}\text { Fuchs } \\
\text { et al } \\
\text { (111) }\end{array}$ & $\begin{array}{l}\text { Nath } \\
\text { et al } \\
\text { (113) }\end{array}$ & $\begin{array}{c}\text { van } \\
\text { Wijk et } \\
\text { al (114) }\end{array}$ & $\begin{array}{c}\text { van } \\
\text { Kleef et } \\
\text { al (115) }\end{array}$ & $\begin{array}{l}\text { Tekin } \\
\text { et al } \\
(116)\end{array}$ & $\begin{array}{l}\text { Civelek } \\
\text { et al } \\
\text { (117) }\end{array}$ & $\begin{array}{l}\text { Dobrogowski } \\
\text { et al (118) }\end{array}$ & $\begin{array}{l}\text { Cohen } \\
\text { et al } \\
(108)\end{array}$ \\
\hline I. & \multicolumn{11}{|c|}{ Trial design and guidance reporting } \\
\hline 1. & Consort or spirit & 3 & 3 & 3 & 3 & 2 & 2 & 2 & 2 & 2 & 3 \\
\hline II. & \multicolumn{11}{|l|}{ Design factors } \\
\hline 2. & Type and design of trial & 2 & 3 & 2 & 3 & 3 & 3 & 3 & 2 & 2 & 2 \\
\hline 3. & Setting/physician & 2 & 1 & 1 & 3 & 3 & 3 & 2 & 2 & 2 & 2 \\
\hline 4. & Imaging & 3 & 3 & 2 & 3 & 3 & 3 & 3 & 3 & 2 & 3 \\
\hline 5. & Sample size & 3 & 2 & 2 & 1 & 1 & 1 & 1 & 1 & 1 & 3 \\
\hline 6. & Statistical methodology & 1 & 1 & 1 & 1 & 1 & 1 & 1 & 1 & 1 & 1 \\
\hline III. & \multicolumn{11}{|l|}{ Patient factors } \\
\hline \multirow[t]{2}{*}{7.} & Inclusiveness of population & & & & & & & & & & \\
\hline & $\begin{array}{l}\text { - For facet or sacroiliac joint } \\
\text { interventions: }\end{array}$ & 2 & 1 & 0 & 2 & 1 & 1 & 1 & 0 & 1 & 1 \\
\hline 8. & Duration of pain & 2 & 2 & 1 & 2 & 2 & 3 & 2 & 0 & 2 & 0 \\
\hline 9. & Previous treatments & 2 & 0 & 0 & 0 & 0 & 0 & 1 & 2 & 0 & 0 \\
\hline 10. & $\begin{array}{l}\text { Duration of follow-up with } \\
\text { appropriate interventions }\end{array}$ & 3 & 2 & 1 & 2 & 2 & 2 & 2 & 2 & 1 & 0 \\
\hline IV. & \multicolumn{11}{|l|}{ Outcomes } \\
\hline 11. & $\begin{array}{l}\text { Outcomes assessment criteria } \\
\text { for significant improvement }\end{array}$ & 4 & 4 & 2 & 4 & 4 & 2 & 2 & 2 & 2 & 0 \\
\hline 12. & $\begin{array}{l}\text { Analysis of all randomized } \\
\text { participants in the groups }\end{array}$ & 2 & 2 & 2 & 2 & 2 & 2 & 2 & 2 & 2 & 2 \\
\hline 13. & Description of drop out rate & 2 & 1 & 2 & 2 & 2 & 2 & 2 & 2 & 2 & 2 \\
\hline 14. & $\begin{array}{l}\text { Similarity of groups at } \\
\text { baseline for important } \\
\text { prognostic indicators }\end{array}$ & 2 & 2 & 2 & 2 & 2 & 2 & 2 & 2 & 2 & 2 \\
\hline 15. & Role of co-interventions & 1 & 0 & 0 & 1 & 1 & 1 & 1 & 1 & 1 & 1 \\
\hline V. & \multicolumn{11}{|l|}{ Randomization } \\
\hline 16. & Method of randomization & 2 & 2 & 2 & 2 & 2 & 2 & 2 & 2 & 2 & 2 \\
\hline VI. & \multicolumn{11}{|l|}{ Allocation concealment } \\
\hline 17. & $\begin{array}{l}\text { Concealed treatment } \\
\text { allocation }\end{array}$ & 2 & 2 & 1 & 2 & 2 & 2 & 2 & 2 & 2 & 0 \\
\hline VII. & \multicolumn{11}{|l|}{ Blinding } \\
\hline 18. & Patient blinding & 1 & 1 & 0 & 1 & 1 & 1 & 1 & 0 & 1 & 0 \\
\hline 19. & Care provider blinding & 1 & 1 & 0 & 1 & 1 & 1 & 1 & 0 & 1 & 0 \\
\hline 20. & Outcome assessor blinding & 0 & 1 & 0 & 0 & 1 & 0 & 0 & 0 & 0 & 0 \\
\hline VIII. & \multicolumn{11}{|l|}{ Conflicts of interest } \\
\hline 21. & Funding and sponsorship & 2 & 3 & 1 & 2 & 0 & 3 & 2 & 0 & 0 & 2 \\
\hline 22. & Conflicts of interest & 3 & 3 & 1 & 3 & 0 & 3 & 2 & 0 & 0 & 2 \\
\hline TOTA & & 45 & 40 & 26 & 42 & 36 & 40 & 37 & 28 & 29 & 28 \\
\hline
\end{tabular}

Source: Manchikanti L, Hirsch JA, Cohen SP, Heavner JE, Falco FJE, Diwan S, Boswell MV, Candido KD, Onyewu O, Zhu J, Sehgal N, Kaye AD, Benyamin RM, Helm II S, Singh V, Datta S, Abdi S, Christo PJ, Hameed H, Hameed M, Vallejo R, Pampati V, Racz GB, Raj PP. Assessment of methodologic quality of randomized trials of interventional techniques: Development of an interventional pain management specific instrument. Pain Physician 2014; 17:E263-E290 (72). 
Pain Physician: July/August 2015; 18:E535-E582

Table 7 (cont.). Methodologic quality assessment of randomized trials of lumbar, cervical and thoracic facet joint interventions utilizing IPM - QRB criteria.

\begin{tabular}{|c|c|c|c|c|c|c|c|c|c|c|c|c|}
\hline & & $\begin{array}{c}\text { Barnsley } \\
\text { et al } \\
\text { (119) }\end{array}$ & $\begin{array}{l}\text { Manchikanti } \\
\text { et al (83) }\end{array}$ & $\begin{array}{l}\text { Lord } \\
\text { et al } \\
\text { (128) }\end{array}$ & $\begin{array}{c}\text { Park } \\
\& \\
\text { Kim } \\
(129)\end{array}$ & $\begin{array}{l}\text { Manchikanti } \\
\text { et al (84) }\end{array}$ & $\begin{array}{c}\text { Joo } \\
\text { et al } \\
(\mathbf{1 2 0})\end{array}$ & $\begin{array}{c}\text { Ribeiro } \\
\text { et al } \\
(121)\end{array}$ & $\begin{array}{l}\text { Moon } \\
\text { et al } \\
(122)\end{array}$ & $\begin{array}{c}\text { Lakemeier } \\
\text { et al } \\
\text { (123) }\end{array}$ & $\begin{array}{c}\text { Yun } \\
\text { et al } \\
(124)\end{array}$ & $\begin{array}{l}\text { Manchikanti } \\
\text { et al (112) }\end{array}$ \\
\hline I. & \multicolumn{12}{|c|}{ Trial design and guidance reporting } \\
\hline 1. & Consort or spirit & 2 & 3 & 3 & 2 & 3 & 2 & 2 & 2 & 2 & 2 & 2 \\
\hline II. & \multicolumn{12}{|l|}{ Design factors } \\
\hline 2. & $\begin{array}{l}\text { Type and design } \\
\text { of trial }\end{array}$ & 2 & 2 & 3 & 2 & 2 & 2 & 2 & 2 & 2 & 2 & 2 \\
\hline 3. & Setting/physician & 2 & 2 & 2 & 2 & 2 & 2 & 2 & 2 & 1 & 1 & 3 \\
\hline 4. & Imaging & 3 & 3 & 3 & 3 & 3 & 3 & 3 & 3 & 3 & 3 & 3 \\
\hline 5. & Sample size & 1 & 3 & 1 & 3 & 3 & 1 & 2 & 2 & 2 & 2 & 2 \\
\hline 6. & $\begin{array}{l}\text { Statistical } \\
\text { methodology }\end{array}$ & 1 & 1 & 1 & 1 & 1 & 1 & 1 & 1 & 1 & 1 & 1 \\
\hline III. & \multicolumn{12}{|l|}{ Patient factors } \\
\hline 7. & $\begin{array}{l}\text { Inclusiveness of } \\
\text { population }\end{array}$ & & & & & & & & & & & \\
\hline & $\begin{array}{l}\text { - For facet or } \\
\text { sacroiliac joint } \\
\text { interventions: }\end{array}$ & 2 & 2 & 2 & 2 & 2 & 2 & 0 & 2 & 1 & 0 & 2 \\
\hline 8. & Duration of pain & 2 & 2 & 2 & 2 & 2 & 2 & 0 & 2 & 2 & 0 & 2 \\
\hline 9. & $\begin{array}{l}\text { Previous } \\
\text { treatments }\end{array}$ & 0 & 2 & 2 & 2 & 2 & 2 & 0 & 2 & 2 & 1 & 2 \\
\hline 10. & $\begin{array}{l}\text { Duration of } \\
\text { follow-up with } \\
\text { appropriate } \\
\text { interventions }\end{array}$ & 1 & 3 & 2 & 2 & 3 & 2 & 1 & 1 & 2 & 1 & 3 \\
\hline IV. & \multicolumn{12}{|l|}{ Outcomes } \\
\hline 11. & $\begin{array}{l}\text { Outcomes } \\
\text { assessment criteria } \\
\text { for significant } \\
\text { improvement }\end{array}$ & 2 & 4 & 4 & 2 & 4 & 2 & 2 & 2 & 2 & 1 & 2 \\
\hline 12. & $\begin{array}{l}\text { Analysis of all } \\
\text { randomized } \\
\text { participants in the } \\
\text { groups }\end{array}$ & 2 & 2 & 2 & 2 & 2 & 2 & 2 & 0 & 2 & 2 & 0 \\
\hline 13. & $\begin{array}{l}\text { Description of } \\
\text { drop out rate }\end{array}$ & 1 & 2 & 2 & 2 & 2 & 2 & 2 & 2 & 2 & 2 & 0 \\
\hline 14. & $\begin{array}{l}\text { Similarity of } \\
\text { groups at baseline } \\
\text { for important } \\
\text { prognostic } \\
\text { indicators }\end{array}$ & 2 & 2 & 2 & 2 & 2 & 2 & 2 & 2 & 2 & 2 & 2 \\
\hline 15. & $\begin{array}{l}\text { Role of } \\
\text { co-interventions }\end{array}$ & 0 & 1 & 1 & 0 & 1 & 1 & 1 & 1 & 1 & 1 & 1 \\
\hline V. & \multicolumn{12}{|l|}{ Randomization } \\
\hline 16. & $\begin{array}{l}\text { Method of } \\
\text { randomization }\end{array}$ & 2 & 2 & 2 & 2 & 2 & 2 & 2 & 2 & 2 & 2 & 0 \\
\hline VI. & \multicolumn{12}{|c|}{ Allocation concealment } \\
\hline 17. & $\begin{array}{l}\text { Concealed } \\
\text { treatment } \\
\text { allocation }\end{array}$ & 2 & 2 & 2 & 0 & 2 & 2 & 2 & 2 & 2 & 2 & 0 \\
\hline VII. & \multicolumn{12}{|l|}{ Blinding } \\
\hline 18. & Patient blinding & 1 & 1 & 1 & 0 & 1 & 1 & 1 & 1 & 1 & 0 & 1 \\
\hline 19. & $\begin{array}{l}\text { Care provider } \\
\text { blinding }\end{array}$ & 1 & 1 & 1 & 0 & 1 & 0 & 0 & 1 & 0 & 0 & 1 \\
\hline
\end{tabular}




\section{Effectiveness of Therapeutic Facet Joint Interventions}

Table 7 (cont.). Methodologic quality assessment of randomized trials of lumbar, cervical and thoracic facet joint interventions utilizing IPM-QRB criteria.

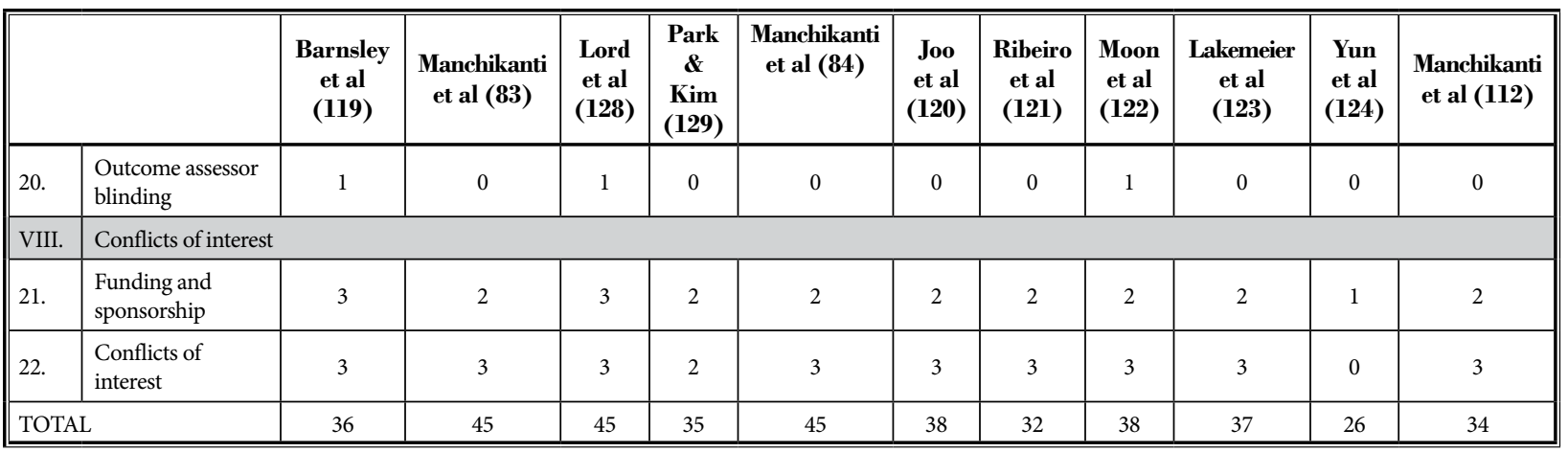

Source: Manchikanti L, Hirsch JA, Cohen SP, Heavner JE, Falco FJE, Diwan S, Boswell MV, Candido KD, Onyewu O, Zhu J, Sehgal N, Kaye AD, Benyamin RM, Helm II S, Singh V, Datta S, Abdi S, Christo PJ, Hameed H, Hameed M, Vallejo R, Pampati V, Racz GB, Raj PP. Assessment of methodologic quality of randomized trials of interventional techniques: Development of an interventional pain management specific instrument. Pain Physician 2014; 17:E263-E290 (72).

Table 8. IPM checklist for assessment of nonrandomized or observational studies of lumbar, cervical and thoracic facet joint interventions of IPM techniques utilizing IPM-QRBNR.

\begin{tabular}{|c|c|c|c|c|c|c|}
\hline & & $\begin{array}{c}\text { Sapir \& } \\
\text { Gorup (159) }\end{array}$ & $\begin{array}{l}\text { MacVicar } \\
\text { et al (160) }\end{array}$ & $\begin{array}{l}\text { Speldewinde } \\
\text { GC (161) }\end{array}$ & $\begin{array}{l}\text { Manchikanti } \\
\text { et al (152) }\end{array}$ & $\begin{array}{l}\text { Manchikanti } \\
\text { et al (162) }\end{array}$ \\
\hline I. & \multicolumn{6}{|l|}{ Study design and guidance reporting } \\
\hline 1. & Strobe or trend guidance & 3 & 3 & 3 & 3 & 3 \\
\hline II. & \multicolumn{6}{|l|}{ Design factors } \\
\hline 2. & Study design and type & 4 & 4 & 4 & 4 & 4 \\
\hline 3. & Setting/physician & 2 & 2 & 2 & 2 & 2 \\
\hline 4. & Imaging & 3 & 3 & 3 & 3 & 3 \\
\hline 5. & Sample size & 2 & 1 & 1 & 1 & 1 \\
\hline 6. & Statistical methodology & 2 & 2 & 2 & 2 & 2 \\
\hline III. & \multicolumn{6}{|l|}{ Patient factors } \\
\hline \multirow[t]{2}{*}{7.} & Inclusiveness of population & & & & & \\
\hline & - For facet or sacroiliac joint interventions: & 4 & 4 & 4 & 4 & 4 \\
\hline 8. & Duration of pain & 2 & 2 & 2 & 2 & 2 \\
\hline 9. & Previous treatments & 2 & 2 & 2 & 2 & 2 \\
\hline 10. & Duration of follow-up with appropriate interventions & 3 & 3 & 3 & 3 & 3 \\
\hline IV. & \multicolumn{6}{|l|}{ Outcomes } \\
\hline 11. & Outcomes assessment criteria for significant improvement & 2 & 4 & 4 & 4 & 4 \\
\hline 12. & Description of drop out rate & 1 & 1 & 1 & 1 & 1 \\
\hline 13. & $\begin{array}{l}\text { Similarity of groups at baseline for important prognostic } \\
\text { indicators }\end{array}$ & 2 & 0 & 0 & 0 & 0 \\
\hline 14. & Role of co-interventions & 2 & 2 & 2 & 2 & 2 \\
\hline V. & \multicolumn{6}{|l|}{ Assignment } \\
\hline 15. & Method of assignment of participants & 4 & 4 & 4 & 2 & 2 \\
\hline VI. & \multicolumn{6}{|l|}{ Conflicts of interest } \\
\hline 16. & Funding and sponsorship & 2 & 1 & 2 & 2 & 2 \\
\hline \multicolumn{2}{|c|}{ TOTAL } & 40 & 38 & 39 & 37 & 37 \\
\hline
\end{tabular}

Source: Manchikanti L, Hirsch JA, Heavner JE, Cohen SP, Benyamin RM, Sehgal N, Falco FJE, Vallejo R, Onyewu O, Zhu J, Kaye AD, Boswell MV, Helm II S, Candido KD, Diwan S, Simopoulos TT, Singh V, Pampati V, Racz GB, Raj PP. Development of an interventional pain management specific instrument for methodologic quality assessment of nonrandomized studies of interventional techniques. Pain Physician 2014; 17:E291-E317 (73). 
among various studies of the 11 trials of radiofrequency neurotomy was performed. None of the 3 trials were homogeneous either for selection criteria, outcome measures, or design of the trial. Among the 5 lumbar intraarticular injections and 3 facet joint nerve blocks there was no clinical homogeneity in any of the 2 or more trials. Thus, a meta-analysis was not feasible; consequently it was not performed.

\subsection{Study Characteristics}

Tables 9 and 10 illustrate the study characteristics of the included studies for randomized trials and observational studies evaluating facet joint interventions.

\subsection{Analysis of Evidence}

The evidence was synthesized based on the modality of treatment for each region. Tables 11 and 12 illustrate the results of therapeutic studies.

A total of 21 randomized trials met inclusion criteria with 9 trials $(108,113-118,122,123)$ evaluating lumbar radiofrequency neurotomy, one trial evaluating cervical radiofrequency neurotomy (128), and one trial evaluating thoracic radiofrequency neurotomy (120); with 3 trials $(82,112,117)$ evaluating therapeutic lumbar facet joint nerve blocks, one trial evaluating therapeutic cervical facet joint nerve blocks (83), and one trial evaluating therapeutic thoracic facet joint nerve blocks (84); and 5 trials evaluating lumbar intraarticular injections $(110,111,121,123,124)$ and 2 trials evaluating cervical intraarticular injections $(119,129)$. In addition, one additional therapeutic thoracic facet joint nerve blocks study (162), one additional therapeutic cervical facet joint nerve block study (152), and 3 additional cervical radiofrequency neurotomy studies (159-161) were also included.

The evidence for radiofrequency neurotomy in the lumbar, cervical, and thoracic spines is variable. The evidence is Level I for short-term effectiveness of radiofrequency neurotomy of less than 6 months and Level II for long-term relief of longer than 6 months based on 8 moderate to high quality randomized controlled trials of radiofrequency neurotomy (108 $, 113,115,116,117,118,122,123)$ showing short-term effectiveness of radiofrequency neurotomy, lack of response shown in one moderate to high quality trial of radiofrequency neurotomy (114), long-term relief based on 3 high quality randomized controlled trials showing effectiveness (115-117), lack of effectiveness demonstrated in one trial (114). In the cervical spine, the evidence is Level II based on one high quality randomized controlled trial (128) for short-term and long-term effectiveness; whereas, in the thoracic spine the evidence is Level III based on one randomized, double-blind, active control trial (120) for short-term and long-term effectiveness.

The evidence for therapeutic facet joint nerve blocks is Level II in the cervical, thoracic, and lumbar regions for short-term and long-term improvement based on 2 high quality randomized controlled trials $(82,117)$ and one moderate (6/12 Cochrane criteria) to high quality (34/48 IPM-QRB criteria) randomized controlled trial (112) of facet joint nerve blocks in the lumbar spine with long-term follow-up $(82,117)$. In the cervical spine, the evidence is Level II based on one high quality randomized controlled trial (83) and the evidence is Level II in the thoracic spine based on one high quality randomized controlled trial (84).

The evidence for intraarticular facet joint injections is variable between the lumbar and cervical spines. There is no evidence available for thoracic intraarticular injections. The evidence for lumbar intraarticular injections of steroids is Level III, based on 3 high quality randomized controlled trials $(121,123,124)$ showing effectiveness with short-term follow-up of less than 6 months and 2 moderate to high quality randomized controlled trials $(110,111)$ showing a lack of effectiveness with a follow-up shorter than 6 months for shortterm pain relief. The evidence for cervical intraarticular injections is Level IV based on one high quality randomized controlled trial (119) showing a lack of effectiveness and one moderate quality randomized controlled trial (129) demonstrating indeterminate results.

\subsection{Discussion}

The systematic review of randomized trials of efficacy of the spinal facet joint interventions in the lumbar, cervical and thoracic regions, with intraarticular injections, facet joint nerve blocks, and radiofrequency neurotomy, revealed variable results. A total of 20 randomized trials were assessed with moderate to high methodologic quality criteria.

Based on the available evidence, there is Level II evidence for lumbar radiofrequency neurotomy, Level II evidence for cervical radiofrequency neurotomy, and Level III evidence for thoracic radiofrequency neurotomy for long-term effectiveness. The evidence is Level II for lumbar, cervical, and thoracic facet joint nerve blocks for long-term effectiveness. For intraarticular injections, the evidence is Level III for lumbar intraarticular injections and Level IV for cervical intraarticular injections; 


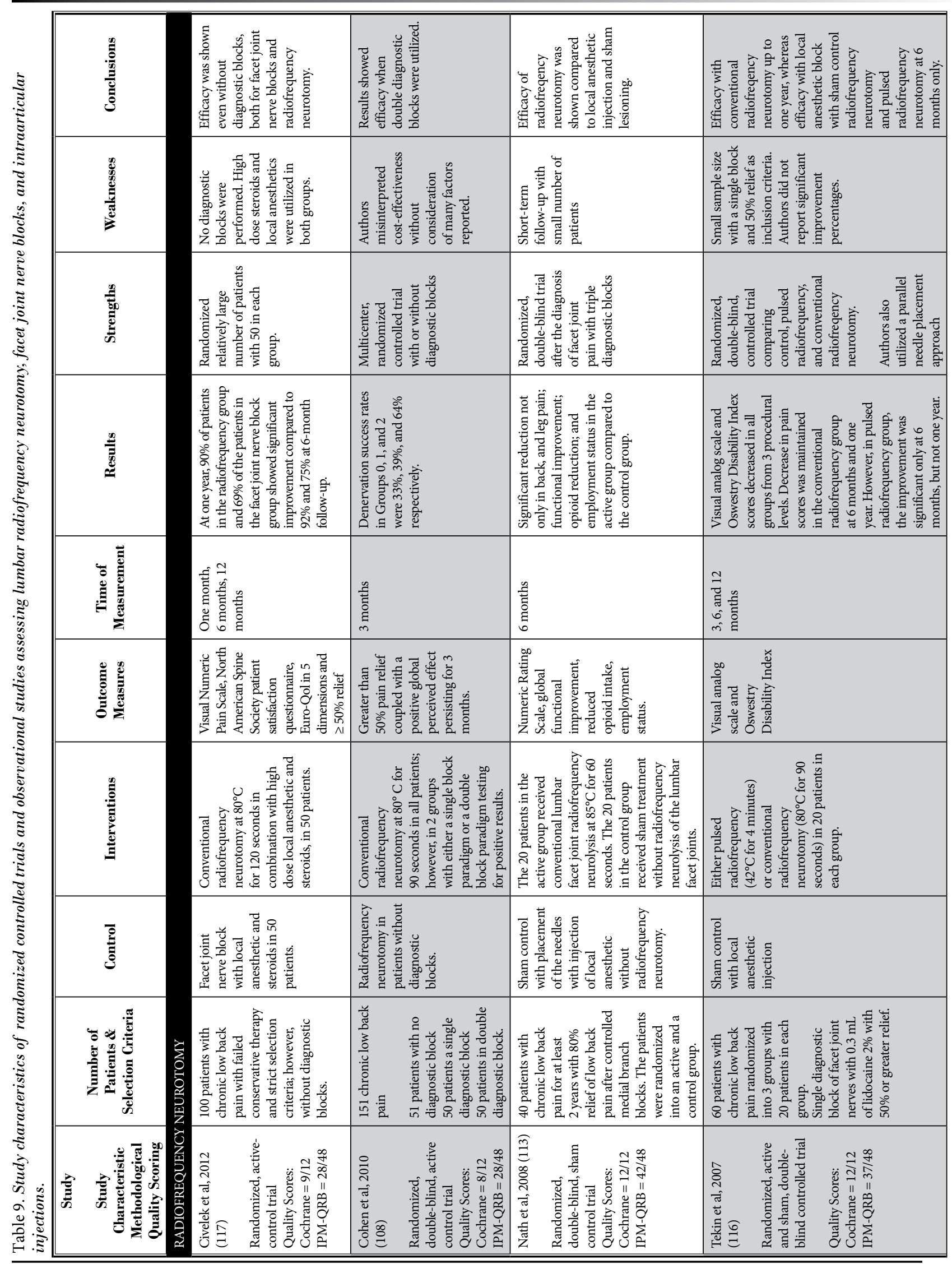


Pain Physician: July/August 2015; 18:E535-E582

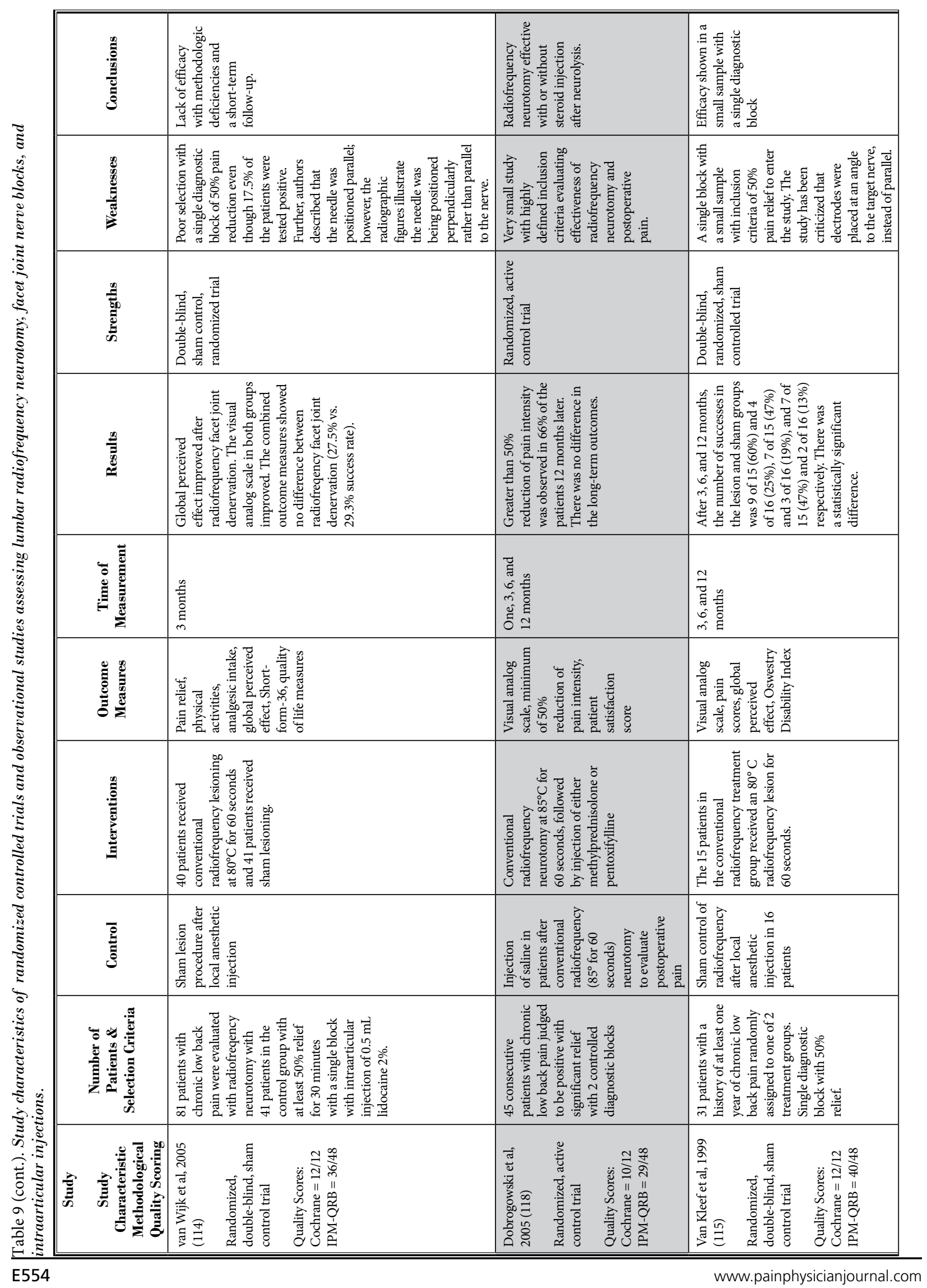


Effectiveness of Therapeutic Facet Joint Interventions

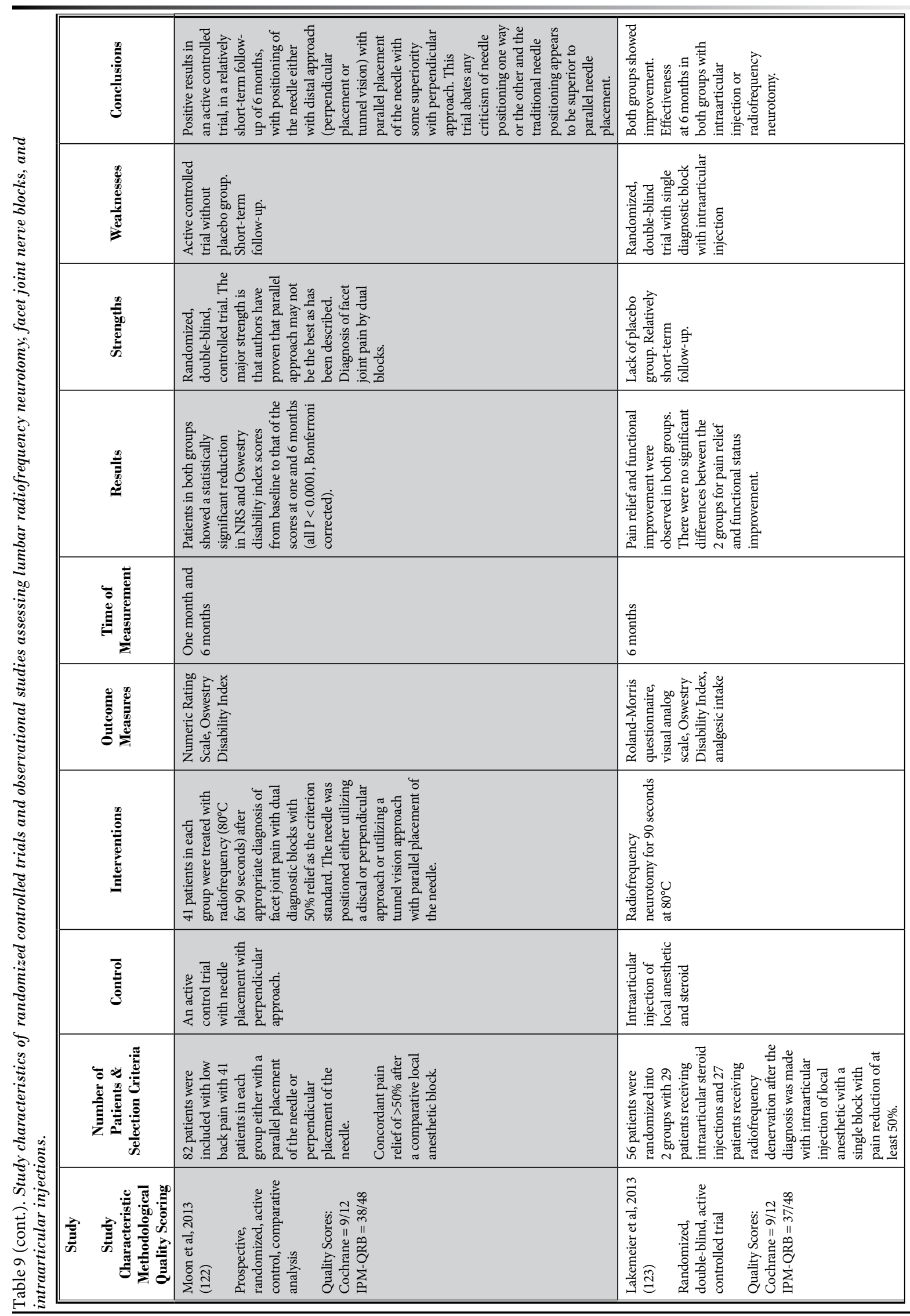

www.painphysicianjournal.com 
Pain Physician: July/August 2015; 18:E535-E582

\begin{tabular}{|c|c|c|c|c|c|}
\hline & 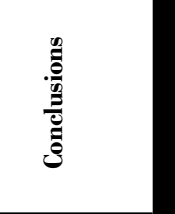 & 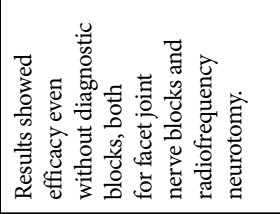 & 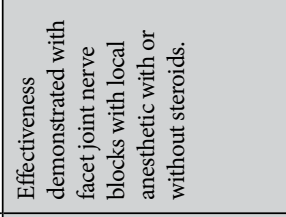 & 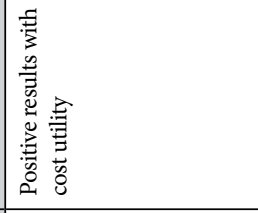 & 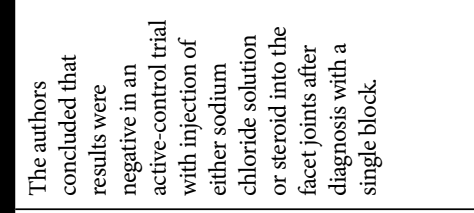 \\
\hline & 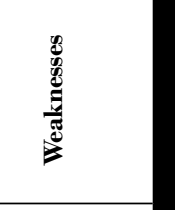 & 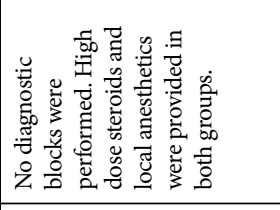 & 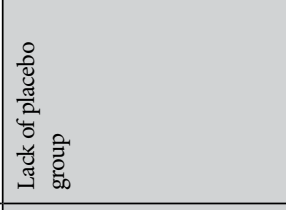 & 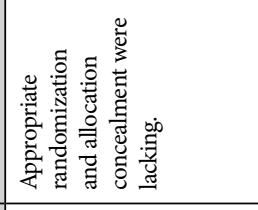 & 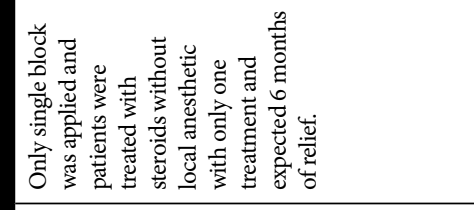 \\
\hline & 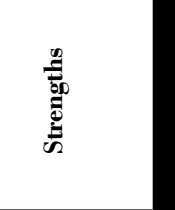 & 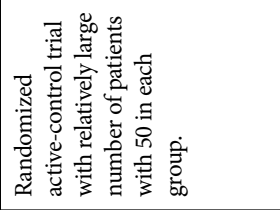 & 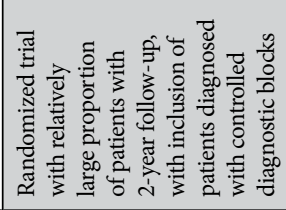 & 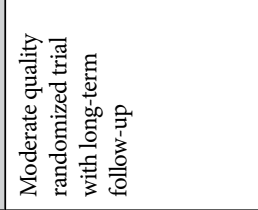 & 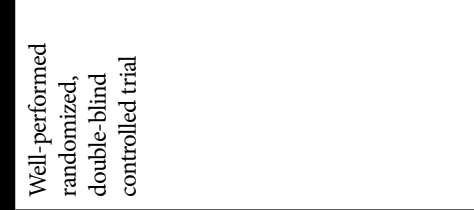 \\
\hline & 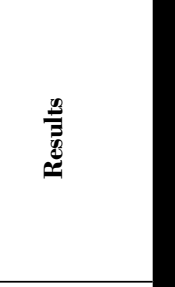 & 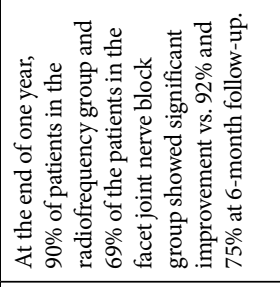 & 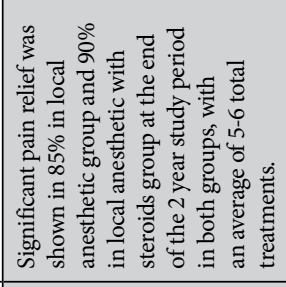 & 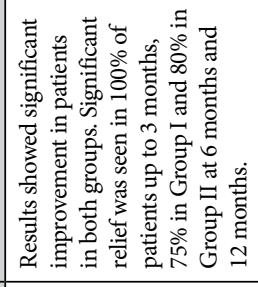 & 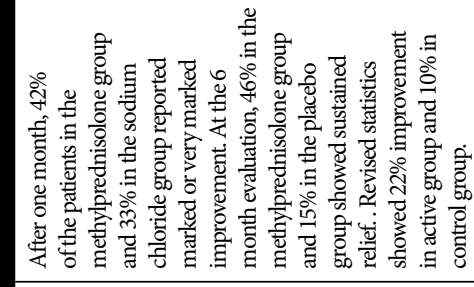 \\
\hline & 竞 & 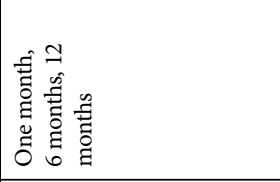 & 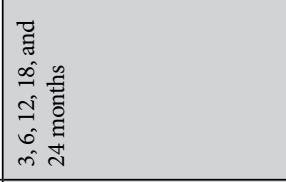 & 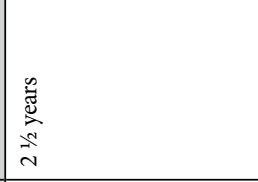 & 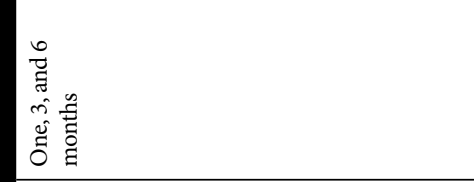 \\
\hline & 递莺 & 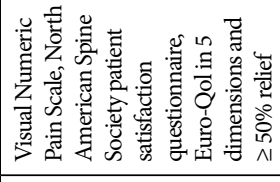 & 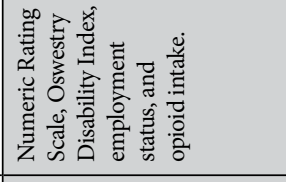 & 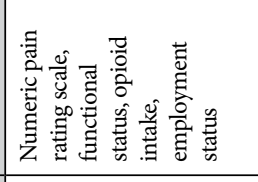 & 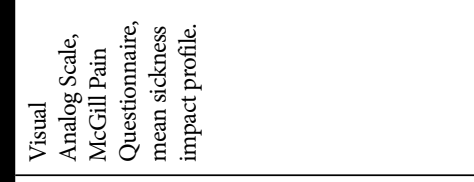 \\
\hline & 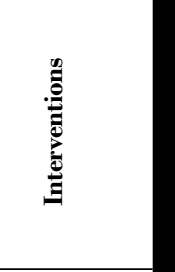 & 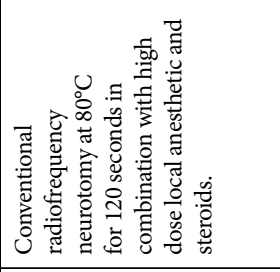 & 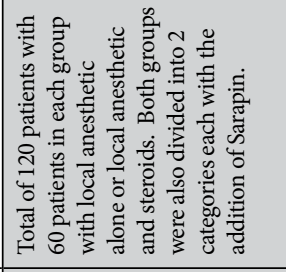 & 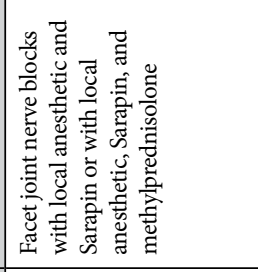 & 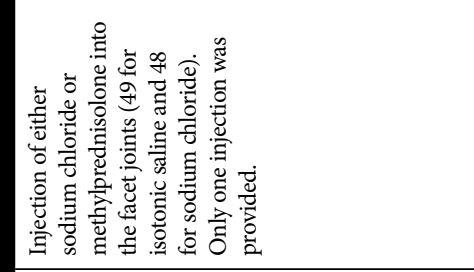 \\
\hline & & 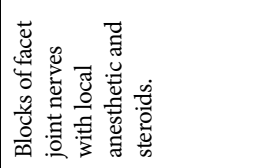 & 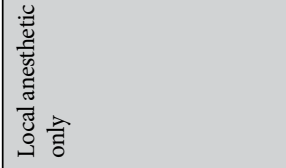 & 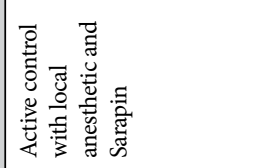 & 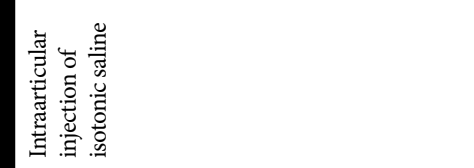 \\
\hline & 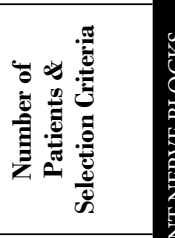 & 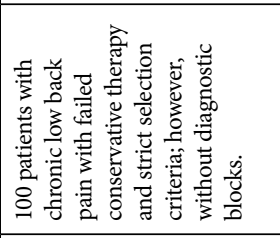 & 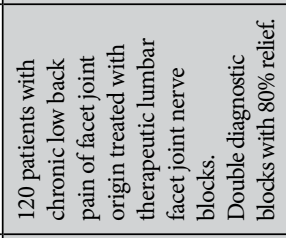 & 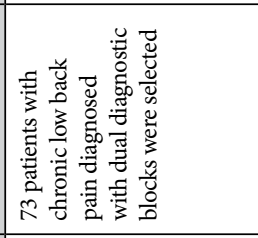 & 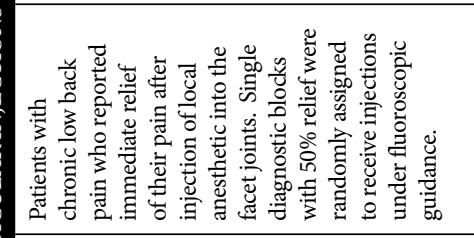 \\
\hline 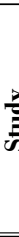 & 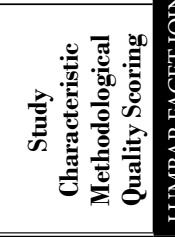 & 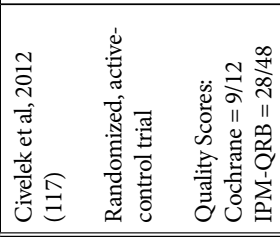 & 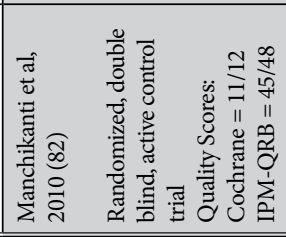 & 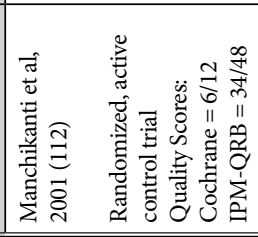 & 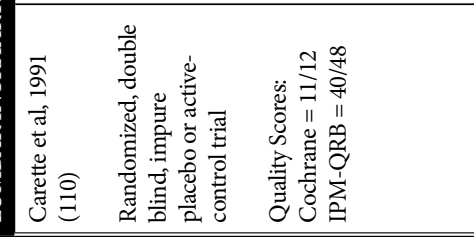 \\
\hline
\end{tabular}




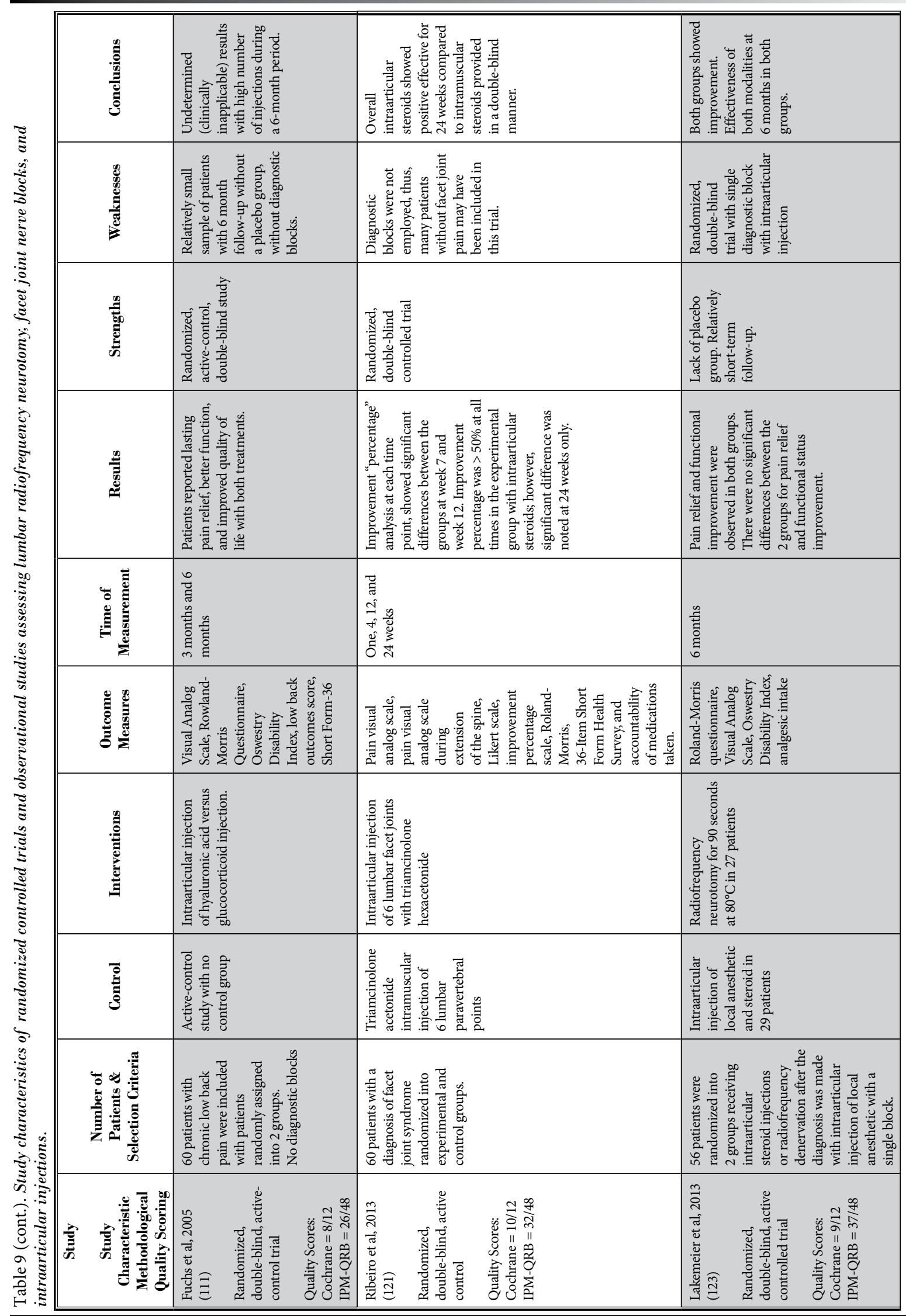




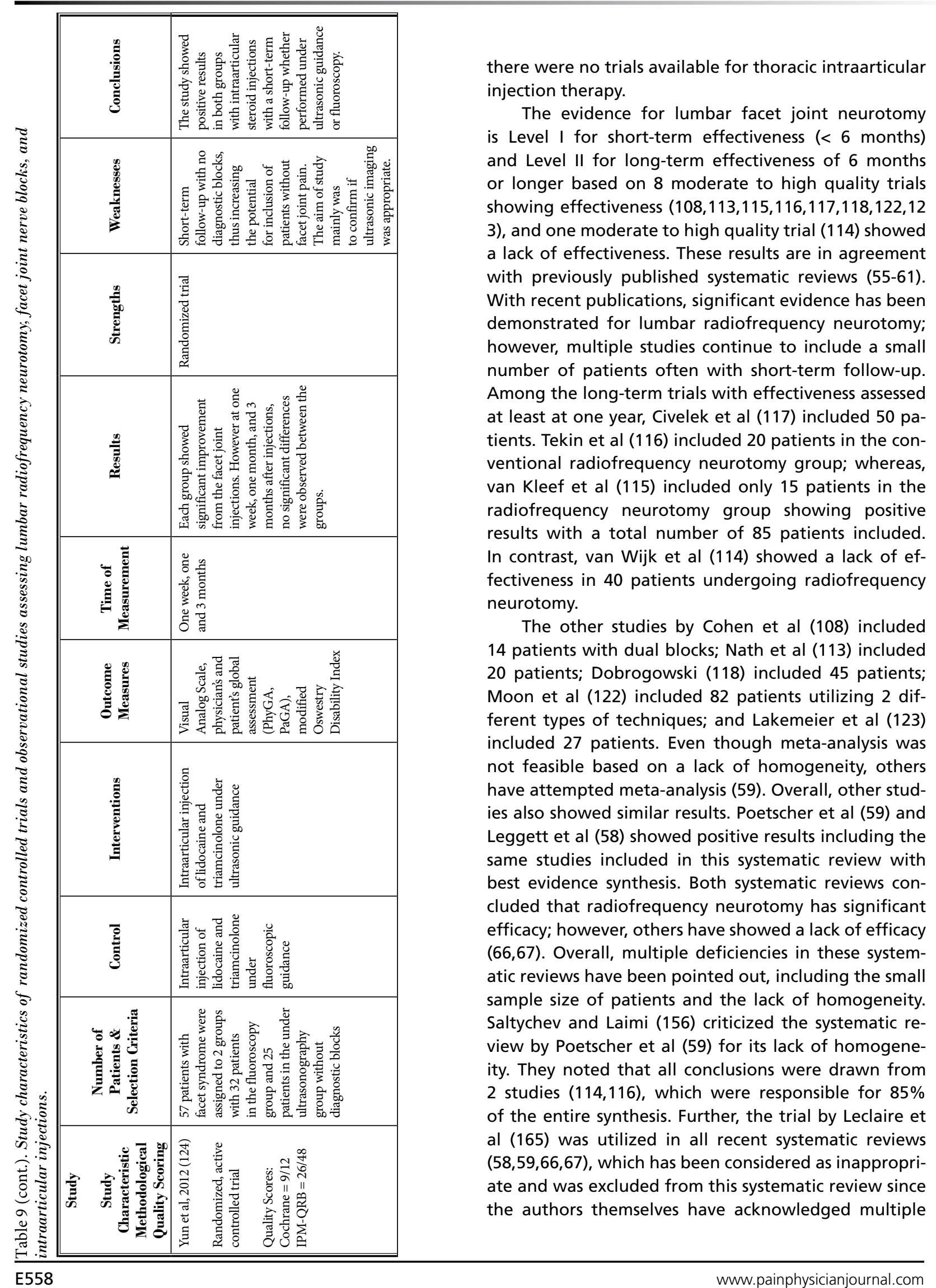


Effectiveness of Therapeutic Facet Joint Interventions

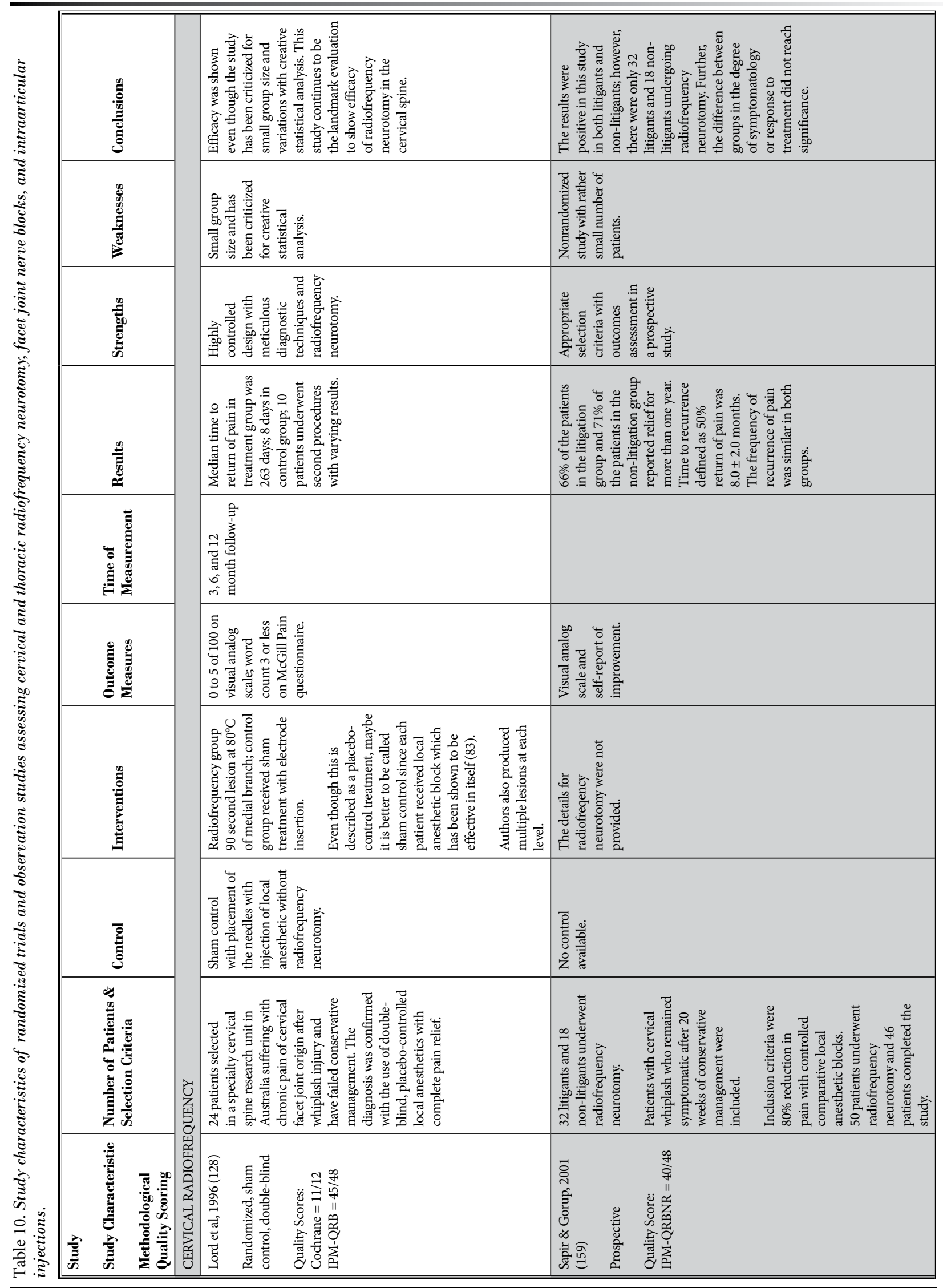

www.painphysicianjournal.com 
Pain Physician: July/August 2015; 18:E535-E582

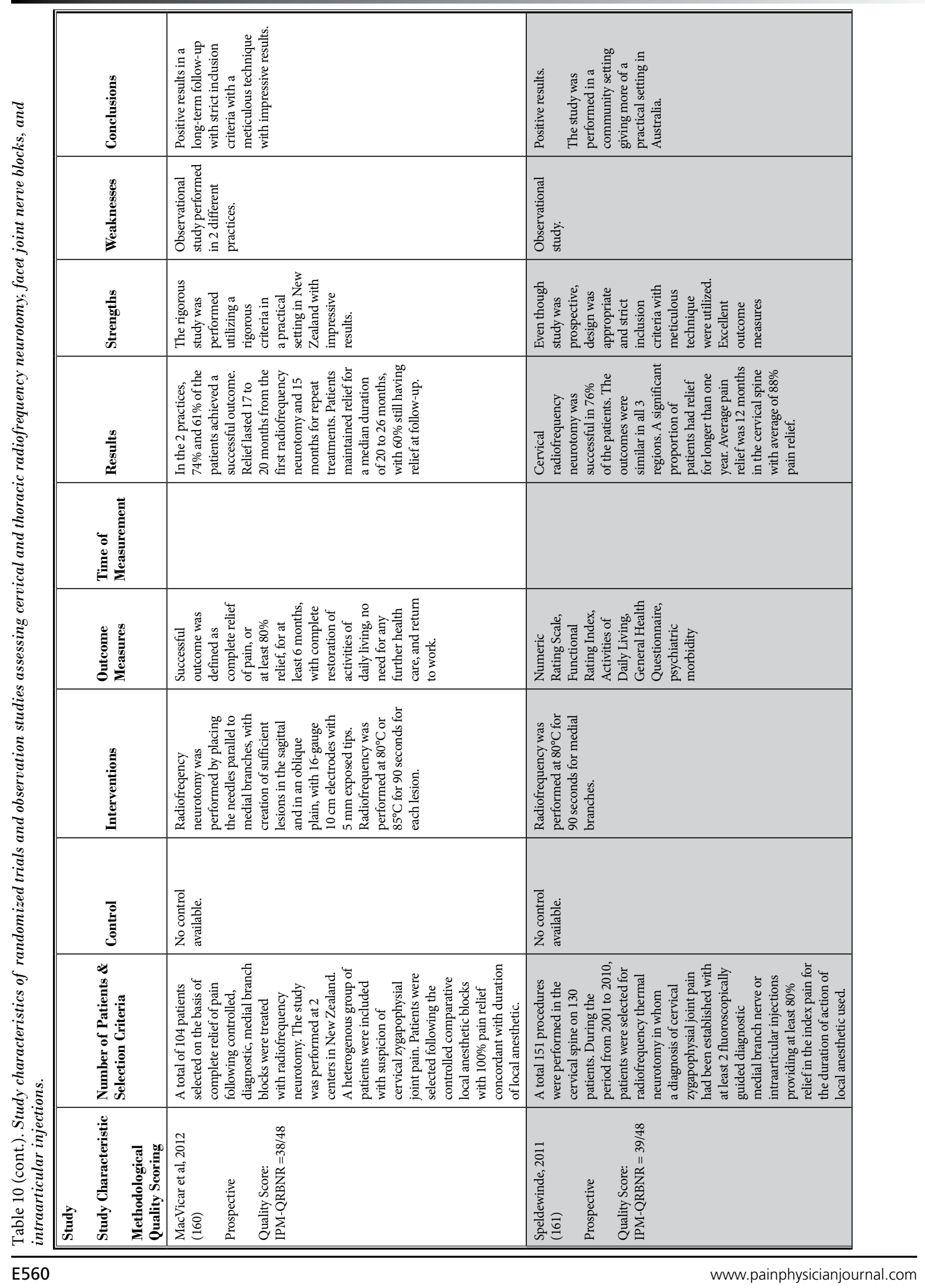




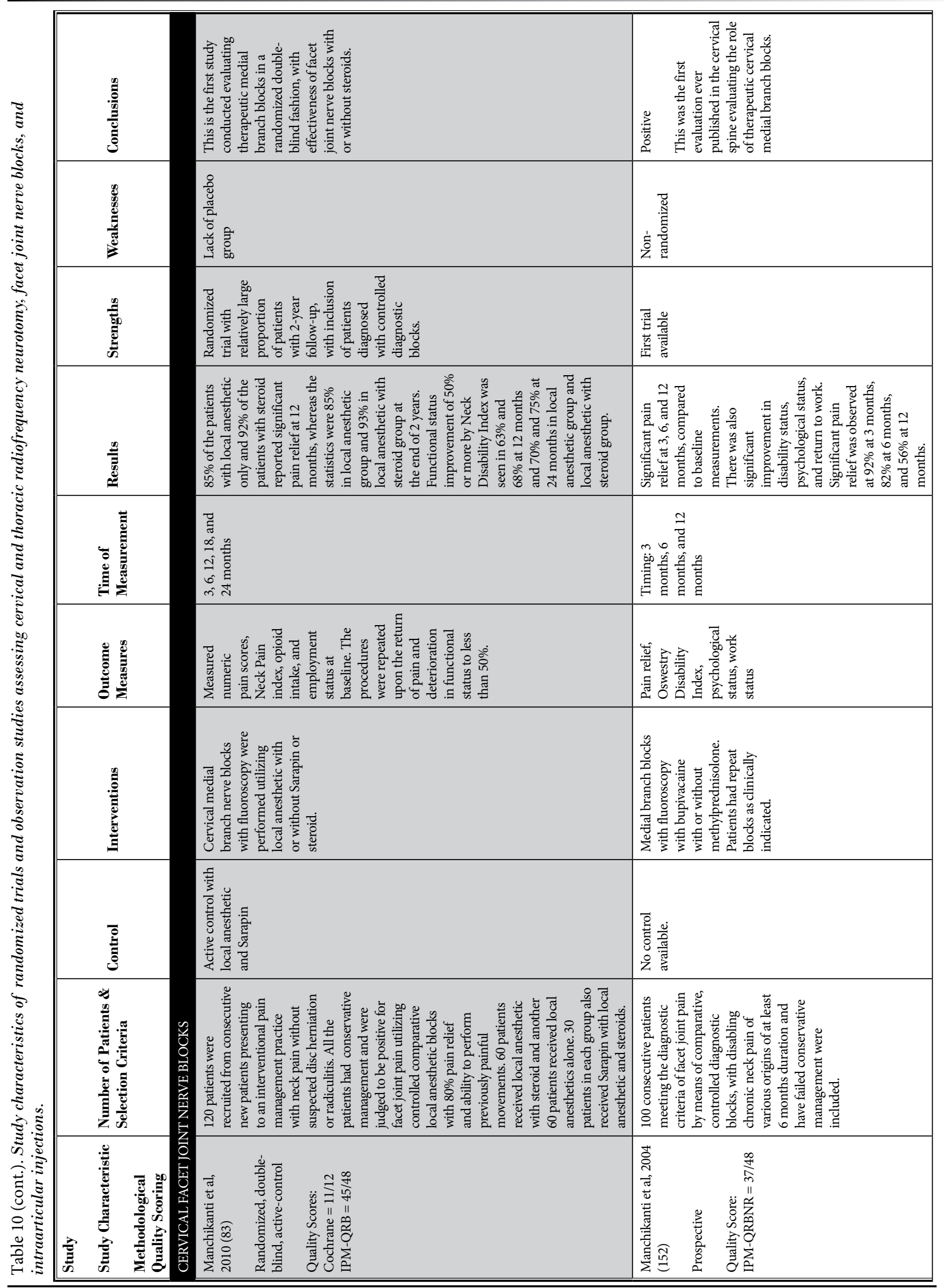


Pain Physician: July/August 2015; 18:E535-E582

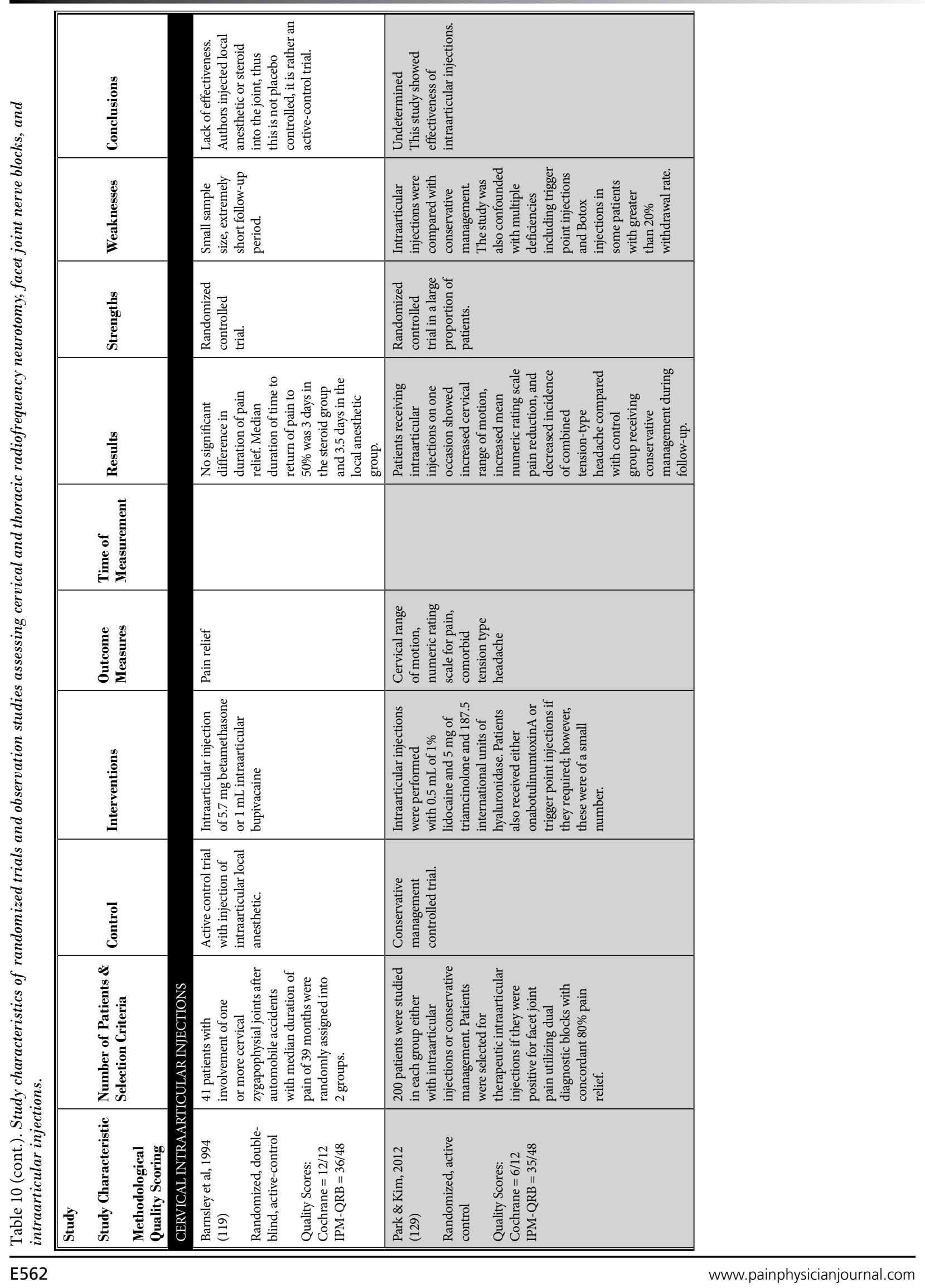




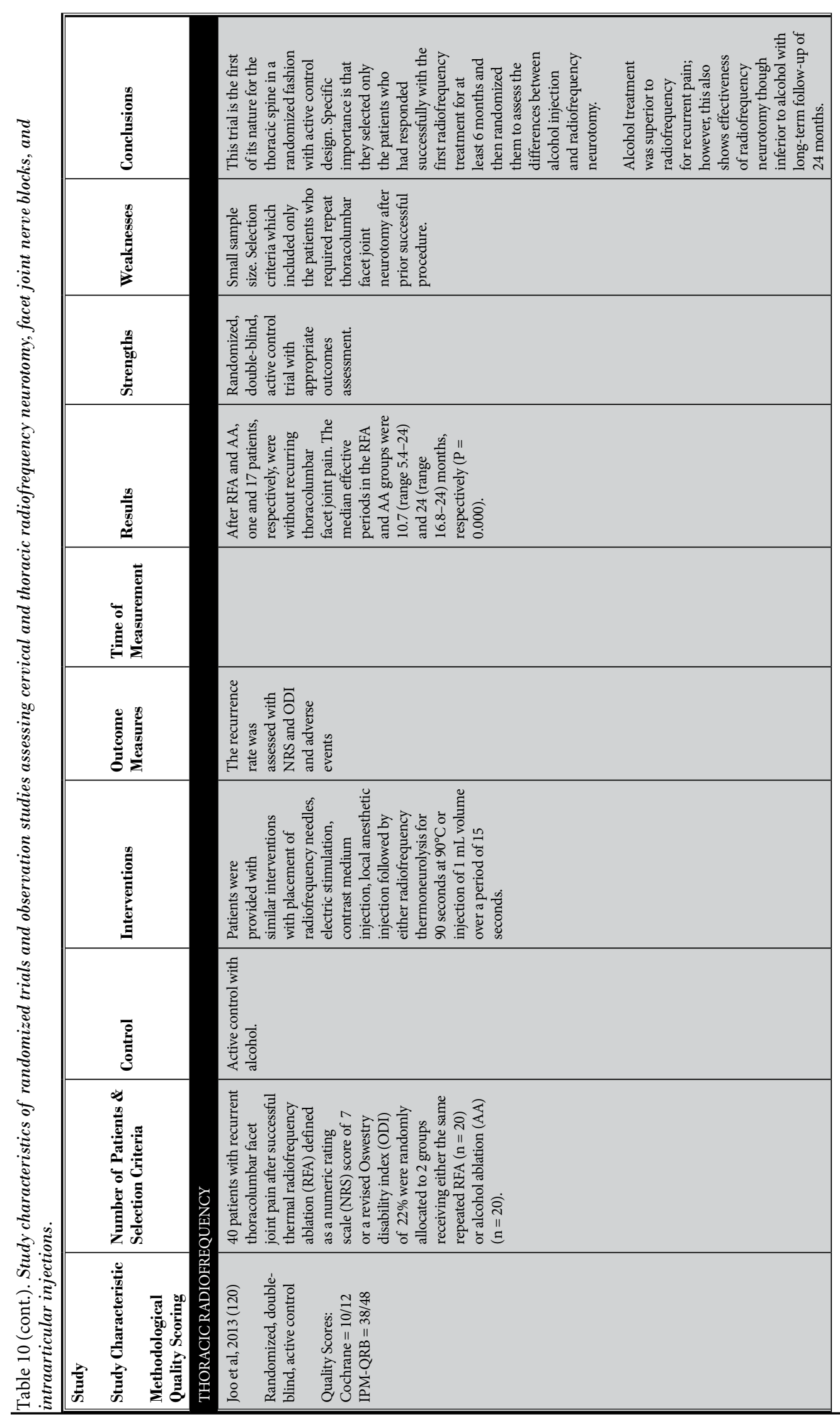




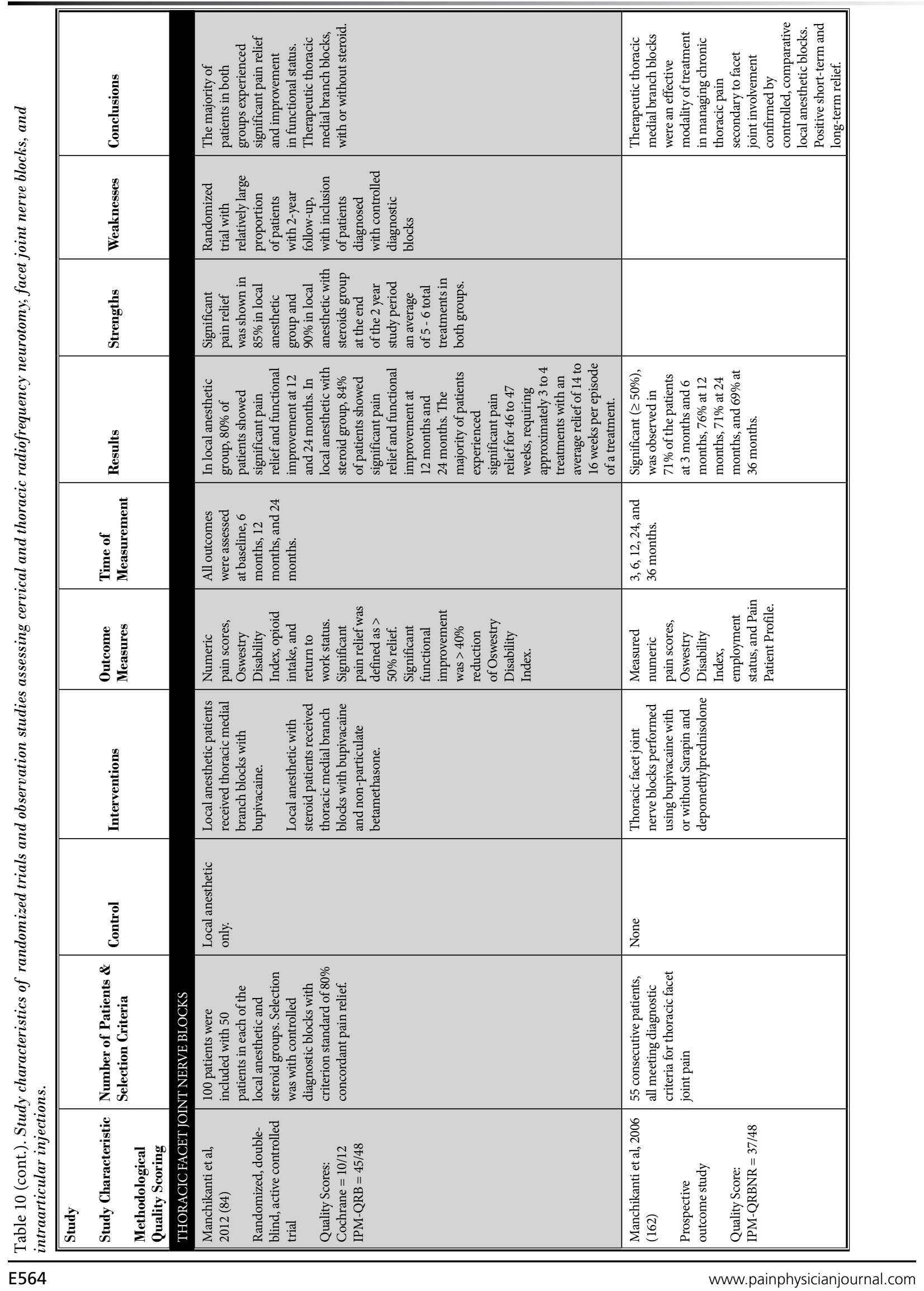


Table 11. Efficacy of lumbar radiofrequency, facet joint nerve blocks, and intraarticular injections.

\begin{tabular}{|c|c|c|c|c|c|c|c|c|c|}
\hline \multirow{3}{*}{$\begin{array}{l}\text { Study } \\
\text { Study } \\
\text { Characteristic } \\
\text { Methodological } \\
\text { Quality Scoring }\end{array}$} & \multirow[t]{3}{*}{ Patients } & \multirow[t]{3}{*}{ Interventions } & \multicolumn{3}{|c|}{ Pain Relief and Function } & \multicolumn{3}{|l|}{ Results } & \multirow[t]{3}{*}{ Comments } \\
\hline & & & \multirow[t]{2}{*}{3 mos. } & \multirow[t]{2}{*}{6 mos. } & \multirow{2}{*}{$\begin{array}{l}12 \\
\text { mos. }\end{array}$} & \multirow{2}{*}{$\begin{array}{l}\text { Short-Term } \\
\leq 6 \mathrm{mos}\end{array}$} & \multicolumn{2}{|l|}{ Long-Term } & \\
\hline & & & & & & & $>6$ mos. & $\geq 1$ year & \\
\hline \multicolumn{10}{|c|}{ LUMBAR RADIOFREQUENCY NEUROTOMY } \\
\hline $\begin{array}{l}\text { Civelek et al, } \\
2012(117) \\
\text { RA, AC } \\
\text { Quality Scores: } \\
\text { Cochrane = } \\
\text { 9/12 } \\
\text { IPM-QRB = } \\
\text { 28/48 }\end{array}$ & 100 & $\begin{array}{l}\mathrm{CRF}=50 \\
\text { Facet joint nerve } \\
\text { blocks }=50\end{array}$ & NA & $92 \%$ vs. $75 \%$ & $\begin{array}{l}90 \% \text { vs. } \\
69 \%\end{array}$ & NA & $\mathrm{P}$ & $\mathrm{P}$ & $\begin{array}{l}\text { Effective for } \\
\text { short and } \\
\text { long-term }\end{array}$ \\
\hline $\begin{array}{l}\text { Cohen et al, } \\
2010(108) \\
\text { RA, DB } \\
\text { Quality Scores: } \\
\text { Cochrane = } \\
8 / 12 \\
\text { IPM-QRB = } \\
\text { 28/48 }\end{array}$ & $\begin{array}{l}\text { "0" block = } 51 \\
\text { One block = } 20 \\
\text { Two blocks } \\
=14\end{array}$ & $\mathrm{CRF}$ & $\begin{array}{l}\text { "0" group = } \\
33 \% \\
\text { One block } \\
=39 \% \\
\text { Two blocks } \\
=64 \%\end{array}$ & NA & NA & $\begin{array}{l}\mathrm{P} \text { in dual block } \\
\text { group }\end{array}$ & NA & NA & $\begin{array}{l}\text { Effective in } \\
\text { short-term } \\
\text { results with } \\
\text { application of } \\
\text { dual blocks } \\
\text { Not effective } \\
\text { with no } \\
\text { or single } \\
\text { diagnostic } \\
\text { blocks. }\end{array}$ \\
\hline $\begin{array}{l}\text { Nath et al, } 2008 \\
\text { (113) } \\
\text { RA, DB, Sham } \\
\text { control } \\
\text { Quality Scores: } \\
\text { Cochrane = } \\
\text { 12/12 } \\
\text { IPM-QRB = } \\
42 / 48\end{array}$ & 40 & $\begin{array}{l}\text { Radiofrequency } \\
=20 \\
\text { Sham }=20\end{array}$ & NA & $\begin{array}{l}\text { Significant } \\
\text { proportion } \\
\text { of patients in } \\
\text { interventional } \\
\text { group }\end{array}$ & NA & $\begin{array}{l}\mathrm{P} \text { for } \\
\text { radiofrequency } \\
\mathrm{N} \text { for sham or } \\
\text { active }\end{array}$ & $\begin{array}{l}\mathrm{P} \text { for } \\
\text { radiofrequency } \\
\mathrm{N} \text { for sham or } \\
\text { active }\end{array}$ & NA & $\begin{array}{l}\text { Effective for } \\
\text { short and } \\
\text { long-term }\end{array}$ \\
\hline $\begin{array}{l}\text { Tekin et al, } 2007 \\
\text { (116) } \\
\text { RA, AC and } \\
\text { sham, DB } \\
\text { Quality Scores: } \\
\text { Cochrane = } \\
12 / 12 \\
\text { IPM-QRB = } \\
37 / 48\end{array}$ & 60 & $\begin{array}{l}\text { CRF }=20 \\
P R F=20 \\
\text { Control }=20\end{array}$ & NA & SI with CRF & $\begin{array}{l}\text { SI with } \\
\text { CRF }\end{array}$ & NA & $\begin{array}{l}\mathrm{P} \text { for } \\
\text { radiofrequency } \\
\mathrm{N} \text { for sham }\end{array}$ & $\begin{array}{l}\mathrm{P} \text { for } \\
\text { radiofrequency } \\
\mathrm{N} \text { for sham }\end{array}$ & $\begin{array}{l}\text { Effective in } \\
\text { long-term }\end{array}$ \\
\hline $\begin{array}{l}\text { van Wijk et al, } \\
2005(114) \\
\text { RA, DB, Sham } \\
\text { control } \\
\text { Quality Scores: } \\
\text { Cochrane = } \\
\text { 12/12 } \\
\text { IPM-QRB = } \\
36 / 48\end{array}$ & 81 & $\begin{array}{l}\text { Radiofrequency = } \\
40 \text { Sham }=41\end{array}$ & $\begin{array}{l}27.5 \% \text { vs. } \\
29.3 \%\end{array}$ & $\begin{array}{l}27.5 \% \text { vs. } \\
29.3 \%\end{array}$ & $\begin{array}{l}27.5 \% \\
\text { vs. } \\
29.3 \%\end{array}$ & $\mathrm{~N}$ & $\mathrm{~N}$ & $\mathrm{~N}$ & $\begin{array}{l}\text { Lack of } \\
\text { effectiveness } \\
\text { with short- } \\
\text { and long-term }\end{array}$ \\
\hline
\end{tabular}


Pain Physician: July/August 2015; 18:E535-E582

Table 11 (cont.). Efficacy of lumbar radiofrequency, facet joint nerve blocks, and intraarticular injections.

\begin{tabular}{|c|c|c|c|c|c|c|c|c|c|}
\hline \multirow{3}{*}{$\begin{array}{l}\text { Study } \\
\text { Study } \\
\text { Characteristic } \\
\text { Methodological } \\
\text { Quality Scoring }\end{array}$} & \multirow[t]{3}{*}{ Patients } & \multirow[t]{3}{*}{ Interventions } & \multicolumn{3}{|c|}{ Pain Relief and Function } & \multicolumn{3}{|l|}{ Results } & \multirow[t]{3}{*}{ Comments } \\
\hline & & & \multirow[t]{2}{*}{3 mos. } & \multirow[t]{2}{*}{6 mos. } & \multirow{2}{*}{$\begin{array}{l}12 \\
\text { mos. }\end{array}$} & \multirow{2}{*}{$\begin{array}{l}\text { Short-Term } \\
\leq 6 \mathrm{mos} \text {. }\end{array}$} & \multicolumn{2}{|l|}{ Long-Term } & \\
\hline & & & & & & & $>6$ mos. & $\geq 1$ year & \\
\hline $\begin{array}{l}\text { Dobrogowski et } \\
\text { al, 2005 (118) } \\
\text { RA, AC } \\
\text { Quality Scores: } \\
\text { Cochrane = } \\
\text { 10/12 } \\
\text { IPM-QRB = } \\
29 / 48\end{array}$ & 45 & CRF & NA & $60 \%$ & NA & NA & $\mathrm{P}$ & NA & $\begin{array}{l}\text { Short- and } \\
\text { long-term } \\
\text { effectiveness }\end{array}$ \\
\hline $\begin{array}{l}\text { Moon et al, } 2013 \\
\text { (122) } \\
\text { Prospective, RA, } \\
\text { comparative } \\
\text { study } \\
\text { Quality Scores: } \\
\text { Cochrane = } \\
\text { 9/12 } \\
\text { IPM-QRB = } \\
\text { 38/48 }\end{array}$ & $\begin{array}{l}\text { Total = } 82 \\
\text { Tunnel vision } \\
\text { approach } \\
\text { group - } 41 \\
\text { patients } \\
\text { included and } \\
34 \text { patients } \\
\text { analyzed. }\end{array}$ & $\begin{array}{l}\text { Radiofrequency } \\
\text { neurotomy distal } \\
\text { approach }\end{array}$ & $\begin{array}{l}\text { SI in both } \\
\text { groups }\end{array}$ & $\begin{array}{l}\text { SI in both } \\
\text { groups }\end{array}$ & NA & $\mathrm{P}$ & $\mathrm{P}$ & NA & $\begin{array}{l}\text { Short- and } \\
\text { long-term } \\
\text { effectiveness }\end{array}$ \\
\hline $\begin{array}{l}\text { Lakemeier et al } \\
\text { (123) } \\
\text { RA, DB } \\
\text { Quality Scores: } \\
\text { Cochrane = } \\
\text { 9/12 } \\
\text { IPM-QRB = } \\
\text { 37/48 }\end{array}$ & $\begin{array}{l}\text { Total }=56 \\
\text { Steroid group } \\
=29 \text { patients } \\
\text { Radiofrequency } \\
\text { group }=27 \\
\text { patients }\end{array}$ & $\begin{array}{l}\text { Intraarticular } \\
\text { lumbar facet joint } \\
\text { steroid injections } \\
\text { compared to } \\
\text { lumbar facet joint } \\
\text { radiofrequency } \\
\text { denervation }\end{array}$ & NA & $\begin{array}{l}\text { SI in both } \\
\text { groups }\end{array}$ & NA & $\mathrm{P}$ & $\mathrm{P}$ & NA & $\begin{array}{l}\text { Short- and } \\
\text { long-term } \\
\text { effectiveness }\end{array}$ \\
\hline \multicolumn{10}{|c|}{ LUMBAR FACET JOINT NERVE BLOCKS } \\
\hline $\begin{array}{l}\text { Civelek et al, } \\
2012 \text { (117) } \\
\text { RA, AC } \\
\text { Quality Scores: } \\
\text { Cochrane = } \\
\text { 9/12 } \\
\text { IPM-QRB = } \\
\text { 28/48 }\end{array}$ & 100 & $\begin{array}{l}\text { LA with steroid } \\
=50 \\
\text { CRF }=50\end{array}$ & NA & $75 \%$ vs. $92 \%$ & $\begin{array}{l}69 \% \text { vs. } \\
90 \%\end{array}$ & NA & $\mathrm{P}$ & $\mathrm{P}$ & $\begin{array}{l}\text { Long-term } \\
\text { effectiveness }\end{array}$ \\
\hline
\end{tabular}


Effectiveness of Therapeutic Facet Joint Interventions

Table 11 (cont.). Efficacy of lumbar radiofrequency, facet joint nerve blocks, and intraarticular injections.

\begin{tabular}{|c|c|c|c|c|c|c|c|c|c|}
\hline \multirow{3}{*}{$\begin{array}{l}\text { Study } \\
\text { Study } \\
\text { Characteristic } \\
\text { Methodological } \\
\text { Quality Scoring }\end{array}$} & \multirow[t]{3}{*}{ Patients } & \multirow[t]{3}{*}{ Interventions } & \multicolumn{3}{|c|}{ Pain Relief and Function } & \multicolumn{3}{|l|}{ Results } & \multirow[t]{3}{*}{ Comments } \\
\hline & & & \multirow[t]{2}{*}{3 mos. } & \multirow[t]{2}{*}{6 mos. } & \multirow{2}{*}{$\begin{array}{l}12 \\
\text { mos. }\end{array}$} & \multirow{2}{*}{$\begin{array}{l}\text { Short-Term } \\
\leq 6 \text { mos. }\end{array}$} & \multicolumn{2}{|c|}{ Long-Term } & \\
\hline & & & & & & & $>6$ mos. & $\geq 1$ year & \\
\hline $\begin{array}{l}\text { Manchikanti et } \\
\text { al, 2010 (82) } \\
\text { RA, DB, AC } \\
\text { Quality Scores: } \\
\text { Cochrane = } \\
11 / 12 \\
\text { IPM-QRB = } \\
45 / 48\end{array}$ & 120 & $\begin{array}{l}\text { LA with steroid } \\
=60 \\
\text { LA }=60\end{array}$ & $\begin{array}{l}82 \% \text { vs. } \\
83 \%\end{array}$ & $93 \%$ vs. $83 \%$ & $\begin{array}{l}85 \% \text { vs. } \\
84 \%\end{array}$ & $\mathrm{P}$ & $\mathrm{P}$ & $\mathrm{P}$ & $\begin{array}{l}\text { Short- and } \\
\text { long-term } \\
\text { effectiveness }\end{array}$ \\
\hline $\begin{array}{l}\text { Manchikanti et } \\
\text { al, } 2001 \text { (112) } \\
\text { RA, AC } \\
\text { Quality Scores: } \\
\text { Cochrane = } \\
\text { 6/12 } \\
\text { IPM-QRB = } \\
34 / 48\end{array}$ & 73 & $\begin{array}{l}\text { LA with steroid } \\
=41 \\
\mathrm{LA}=32\end{array}$ & $\begin{array}{l}100 \% \text { vs } \\
100 \%\end{array}$ & $75 \%$ vs $80 \%$ & $\begin{array}{l}75 \% \text { vs } \\
80 \%\end{array}$ & $\mathrm{P}$ & $\mathrm{P}$ & $\mathrm{P}$ & $\begin{array}{l}\text { Positive short } \\
\text { and long-term } \\
\text { results }\end{array}$ \\
\hline \multicolumn{10}{|c|}{ LUMBAR INTRAARTICULAR INJECTIONS } \\
\hline $\begin{array}{l}\text { Carette et al, } \\
1991(110) \\
\text { RA, DB, PC } \\
\text { or AC } \\
\text { Quality Scores: } \\
\text { Cochrane = } \\
11 / 12 \\
\text { IPM-QRB = } \\
40 / 48\end{array}$ & 97 & $\begin{array}{l}\text { Methylprednisolone } \\
\text { acetate }=49 \\
\text { Isotonic saline }=48 \\
\text { patients }\end{array}$ & $\begin{array}{l}33 \% \text { vs. } \\
42 \%\end{array}$ & $22 \%$ vs. $10 \%$ & NA & $\mathrm{N}$ & $\mathrm{N}$ & NA & $\begin{array}{l}\text { Lack of } \\
\text { effectiveness }\end{array}$ \\
\hline $\begin{array}{l}\text { Fuchs et al, } 2005 \\
\text { (111) } \\
\text { R, DB, AC } \\
\text { Quality Scores: } \\
\text { Cochrane = } \\
8 / 12 \\
\text { IPM-QRB = } \\
26 / 48\end{array}$ & 60 & $\begin{array}{l}\text { Hyaluronic } \\
\text { acid versus } \\
\text { glucocorticoid with } \\
6 \text { injections. }\end{array}$ & $\begin{array}{l}\text { Significant } \\
\text { proportion } \\
\text { of patients }\end{array}$ & $\begin{array}{l}\text { Significant } \\
\text { proportion } \\
\text { of patients }\end{array}$ & NA & $\mathrm{U}$ & $\mathrm{U}$ & NA & $\begin{array}{l}\text { Effectiveness } \\
\text { undetermined }\end{array}$ \\
\hline $\begin{array}{l}\text { Ribeiro et al, } \\
2013(121) \\
\text { RA, DB, AC } \\
\\
\text { Quality Scores: } \\
\text { Cochrane = } \\
10 / 12 \\
\text { IPM-QRB = } \\
32 / 48\end{array}$ & 60 & $\begin{array}{l}\text { Intraarticular } \\
\text { injection group }=31 \\
\text { Intramuscular } \\
\text { steroid injection } \\
\text { group }=29\end{array}$ & $52 \%$ vs $45 \%$ & $55 \%$ vs $38 \%$ & NA & $\mathrm{P}$ & $\mathrm{P}$ & NA & $\begin{array}{l}\text { Short- and } \\
\text { long-term } \\
\text { effectiveness }\end{array}$ \\
\hline
\end{tabular}


Table 11 (cont.). Efficacy of lumbar radiofrequency, facet joint nerve blocks, and intraarticular injections.

\begin{tabular}{|c|c|c|c|c|c|c|c|c|c|}
\hline \multirow{3}{*}{$\begin{array}{l}\text { Study } \\
\text { Study } \\
\text { Characteristic } \\
\text { Methodological } \\
\text { Quality Scoring } \\
\end{array}$} & \multirow[t]{3}{*}{ Patients } & \multirow[t]{3}{*}{ Interventions } & \multicolumn{3}{|c|}{ Pain Relief and Function } & \multicolumn{3}{|l|}{ Results } & \multirow[t]{3}{*}{ Comments } \\
\hline & & & \multirow[t]{2}{*}{3 mos. } & \multirow[t]{2}{*}{6 mos. } & \multirow{2}{*}{$\begin{array}{l}12 \\
\text { mos. }\end{array}$} & \multirow{2}{*}{$\begin{array}{l}\text { Short-Term } \\
\leq 6 \text { mos. }\end{array}$} & \multicolumn{2}{|c|}{ Long-Term } & \\
\hline & & & & & & & $>6 \mathrm{mos}$ & $\geq 1$ year & \\
\hline $\begin{array}{l}\text { Yun et al, } 2012 \\
(124) \\
\text { RA } \\
\text { Quality Scores: } \\
\text { Cochrane = } \\
\text { 9/12 } \\
\text { IPM-QRB = } \\
26 / 48\end{array}$ & $\begin{array}{l}\text { Total }=57 \\
\text { Fluoroscopy } \\
\text { group }=32 \\
\text { Ultrasonography } \\
\text { group }=25\end{array}$ & $\begin{array}{l}\text { Intraarticular } \\
\text { injection of local } \\
\text { anesthetic and } \\
\text { steroid }\end{array}$ & $\begin{array}{l}\text { SI in both } \\
\text { groups }\end{array}$ & NA & NA & $P$ & NA & NA & $\begin{array}{l}\text { Short-term } \\
\text { effectiveness }\end{array}$ \\
\hline $\begin{array}{l}\text { Lakemeier et al, } \\
2013 \text { (123) } \\
\text { RA, DB } \\
\text { Quality Scores: } \\
\text { Cochrane = } \\
\text { 9/12 } \\
\text { IPM-QRB = } \\
\text { 37/48 }\end{array}$ & $\begin{array}{l}\text { Total }=56 \\
\text { Steroid group }= \\
29 \text { patients } \\
\text { Radiofrequency } \\
\text { group }=27 \\
\text { patients }\end{array}$ & $\begin{array}{l}\text { Intraarticular } \\
\text { lumbar facet joint } \\
\text { steroid injections } \\
\text { compared to } \\
\text { lumbar facet joint } \\
\text { radiofrequency } \\
\text { denervation }\end{array}$ & NA & $\begin{array}{l}\text { SI in both } \\
\text { groups }\end{array}$ & NA & $\mathrm{P}$ & $\mathrm{P}$ & NA & $\begin{array}{l}\text { Short-and } \\
\text { long-term } \\
\text { effectiveness }\end{array}$ \\
\hline
\end{tabular}

$\mathrm{RA}=$ randomized $\mathrm{DB}=$ double-blind; $\mathrm{AC}=$ active control; $\mathrm{ST}=$ steroid; $\mathrm{LA}=$ local anesthetic; $\mathrm{SAL}=$ saline; $\mathrm{SI}=$ significant improvement; $\mathrm{P}=$ positive; $\mathrm{N}$ = negative; $\mathrm{NA}$ = not applicable

flaws in performing their trial. Further, multiple issues related to radiofrequency neurotomy and exclusion of studies is based on an author's own criteria such, as by Leclaire et al (165).

The evidence for cervical radiofrequency neurotomy was derived from only one high quality randomized controlled trial with an extremely small sample size of patients (128). There were no other trials; consequently, it appears that the level of evidence of II or III may be appropriate. Since there was only one randomized double-blind controlled trial, it may be worthwhile to look at the evidence from nonrandomized prospective trials. The randomized trial by Lord et al (128) included 24 patients and compared percutaneous radiofrequency neurotomy to a sham procedure. Thus, the technique of radiofrequency thermoneurolysis was the same, but radiofrequency lesioning was not performed in the sham control group.

The inclusion criteria were rather strict with comparative local anesthetic blocks with $100 \%$ concordant pain relief. The post-treatment assessment was carried out with the Visual Analog Scale and the McGill Pain Questionnaire. The results showed one patient in the sham control group and 7 patients in the active treatment group were pain-free at the 27-week follow-up.
Their results also showed that the median time for return of pain to at least $50 \%$ of the preoperative level was 263 days in the active treatment groups; whereas it was 8 days in the sham control group. The authors concluded that this trial proved that radiofrequency neurotomy is capable of giving pain relief for up to and over a year. Even though this study was meticulously performed in an academic setting, it only included a small number of patients with whiplash injury and the technique with multiple lesions is not commonly utilized in the United States. This trial also faced significant criticism by Carragee et al (157) for multiple aspects that have not been widely recognized and criticized in the past, which included the differences in baseline characteristics of patients among both groups and the nature of blinding. In fact, Carragee et al (157) reported that the integrity of the blinding was in doubt related to the fact that $42 \%$ of the active group developed long-term anesthetic or dysesthetic areas of the skin, whereas none of the patients in the sham control group developed changes. Thus, Carragee et al (157) felt that there was no significant blinding technique applied with ability of the participants to recognize their group assignment. In addition, litigation also had no significant difference in the outcomes. Overall, the 
Table 12. Results of randomized trials of cervical and thoracic radiofreqency neurotomy, facet joint, nerve blocks and intraarticular injections.

\begin{tabular}{|c|c|c|c|c|c|c|c|c|c|}
\hline \multirow{3}{*}{$\begin{array}{l}\text { Study } \\
\text { Study Characteristic } \\
\text { Methodological } \\
\text { Quality Scoring }\end{array}$} & \multirow[t]{3}{*}{ Patients } & \multirow[t]{3}{*}{ Interventions } & \multicolumn{3}{|c|}{ Pain Relief and Function } & \multicolumn{3}{|l|}{ Results } & \multirow[t]{3}{*}{ Comments } \\
\hline & & & \multirow[t]{2}{*}{3 mos. } & \multirow[t]{2}{*}{$6 \mathrm{mos}}$. & \multirow[t]{2}{*}{12 mos. } & \multirow{2}{*}{$\begin{array}{l}\text { Short-Term } \\
\leq 6 \text { mos. }\end{array}$} & \multicolumn{2}{|c|}{ Long-Term } & \\
\hline & & & & & & & $>6$ mos. & $\geq 1$ year & \\
\hline \multicolumn{10}{|c|}{ CERVICAL RADIOFREQUENCY } \\
\hline $\begin{array}{l}\text { Lord et al, } 1996 \text { (128) } \\
\text { RA, sham control, DB } \\
\text { Quality Scores: } \\
\text { Cochrane = 11/12 } \\
\text { IPM-QRB = 45/48 }\end{array}$ & 24 & $\begin{array}{l}\text { Conventional RFTN } \\
80^{\circ} \mathrm{C}, 90 \text { seconds } \\
\text { Sham }=12 \\
\text { Intervention }=12\end{array}$ & NA & $\begin{array}{l}\text { One of } \\
\text { sham } \\
7 \text { of } \\
\text { active }\end{array}$ & $\begin{array}{l}58 \% \text { in } \\
\text { active } \\
\text { treatment } \\
\text { group }\end{array}$ & $\mathrm{P}$ & $\mathrm{P}$ & $\mathrm{P}$ & $\begin{array}{l}\text { Short-and } \\
\text { long-term } \\
\text { effectiveness }\end{array}$ \\
\hline $\begin{array}{l}\text { Sapir \& Gorup, } 2001 \\
\text { (159) } \\
\text { Prospective } \\
\text { Quality Score: } \\
\text { IPM-QRBNR = 40/48 }\end{array}$ & 50 & $\begin{array}{l}\text { Conventional RFTN } \\
80^{\circ} \mathrm{C}, 90 \text { seconds } \\
\text { Litigants }=32 \\
\text { Non-litigants }=18\end{array}$ & NA & NA & $\begin{array}{l}66 \% \\
\text { litigant } \\
71 \% \\
\text { non-litigant }\end{array}$ & NA & NA & $\mathrm{P}$ & $\begin{array}{l}\text { Long-term } \\
\text { effectiveness }\end{array}$ \\
\hline $\begin{array}{l}\text { MacVicar et al, } 2012 \\
(160) \\
\text { Prospective } \\
\text { Quality Score: } \\
\text { IPM-QRBNR =38/48 }\end{array}$ & 104 & $\begin{array}{l}\text { Conventional RFTN } \\
80^{\circ} \mathrm{C}, 90 \text { seconds } \\
2 \text { practices }\end{array}$ & NA & NA & $\begin{array}{l}74 \% \text { vs } \\
61 \%\end{array}$ & NA & NA & $\mathrm{P}$ & $\begin{array}{l}\text { Long-term } \\
\text { effectiveness }\end{array}$ \\
\hline $\begin{array}{l}\text { Speldewinde, } 2011 \\
\text { (161) } \\
\text { Prospective } \\
\text { Quality Score: } \\
\text { IPM-QRBNR = 39/48 }\end{array}$ & 130 & $\begin{array}{l}\text { Conventional RFTN } \\
80^{\circ} \mathrm{C}, 90 \text { seconds }\end{array}$ & NA & NA & $76 \%$ & NA & NA & $\mathrm{P}$ & $\begin{array}{l}\text { Long-term } \\
\text { effectiveness }\end{array}$ \\
\hline \multicolumn{10}{|c|}{ CERVICAL FACET JOINT NERVE BLOCKS } \\
\hline $\begin{array}{l}\text { Manchikanti et al, } 2010 \\
(83) \\
\text { RA, DB, AC } \\
\text { Quality Scores: } \\
\text { Cochrane = } 11 / 12 \\
\text { IPM-QRB = 45/48 }\end{array}$ & 120 & $\begin{array}{l}\text { Local anesthetic }=60 \\
\text { Local anesthetic with } \\
\text { steroid }=60\end{array}$ & $\begin{array}{l}83 \% \\
\text { versus } \\
85 \%\end{array}$ & $\begin{array}{l}87 \% \\
\text { versus } \\
95 \%\end{array}$ & $\begin{array}{l}85 \% \text { versus } \\
92 \%\end{array}$ & $\mathrm{P}$ & $\mathrm{P}$ & $\mathrm{P}$ & $\begin{array}{l}\text { Short- and } \\
\text { long-term } \\
\text { effectiveness }\end{array}$ \\
\hline $\begin{array}{l}\text { Manchikanti et al, } 2004 \\
\text { (152) } \\
\text { Prospective } \\
\text { Quality Score: } \\
\text { IPM-QRBNR = 37/48 }\end{array}$ & 100 & $\begin{array}{l}\text { Therapeutic medical } \\
\text { branch blocks }\end{array}$ & $92 \%$ & $82 \%$ & $56 \%$ & $\mathrm{P}$ & $\mathrm{P}$ & $\mathrm{P}$ & $\begin{array}{l}\text { Long-term } \\
\text { effectiveness }\end{array}$ \\
\hline \multicolumn{10}{|c|}{ CERVICAL INTRAARTICULAR INJECTIONS } \\
\hline $\begin{array}{l}\text { Barnsley et al, } 1994 \\
(119) \\
\text { RA, DB, AC } \\
\text { Quality Scores: } \\
\text { Cochrane = 12/12 } \\
\text { IPM-QRB = 36/48 }\end{array}$ & 41 & $\begin{array}{l}\mathrm{LA}=20 \\
\text { Steroid }=21\end{array}$ & $20 \%$ & $20 \%$ & $20 \%$ & $\mathrm{~N}$ & $\mathrm{~N}$ & $\mathrm{~N}$ & $\begin{array}{l}\text { Lack of } \\
\text { effectiveness }\end{array}$ \\
\hline
\end{tabular}


Table 12 (cont.). Results of randomized trials of cervical and thoracic radiofreqency neurotomy, facet joint, nerve blocks and intraarticular injections.

\begin{tabular}{|c|c|c|c|c|c|c|c|c|c|}
\hline \multirow{3}{*}{$\begin{array}{l}\text { Study } \\
\text { Study Characteristic } \\
\text { Methodological } \\
\text { Quality Scoring }\end{array}$} & \multirow[t]{3}{*}{ Patients } & \multirow[t]{3}{*}{ Interventions } & \multicolumn{3}{|c|}{ Pain Relief and Function } & \multicolumn{3}{|l|}{ Results } & \multirow[t]{3}{*}{ Comments } \\
\hline & & & \multirow[t]{2}{*}{3 mos. } & \multirow[t]{2}{*}{6 mos. } & \multirow[t]{2}{*}{12 mos. } & \multirow{2}{*}{$\begin{array}{l}\text { Short-Term } \\
\leq 6 \text { mos. }\end{array}$} & \multicolumn{2}{|c|}{ Long-Term } & \\
\hline & & & & & & & $>6$ mos. & $\geq 1$ year & \\
\hline $\begin{array}{l}\text { Park \& Kim, } 2012 \text { (129) } \\
\text { RA, AC } \\
\text { Quality Scores: } \\
\text { Cochrane = 6/12 } \\
\text { IPM-QRB = 35/48 }\end{array}$ & 306 & $\begin{array}{l}\text { Non-injection group } \\
=151 \\
\text { Nerve blocks = } 155\end{array}$ & $\mathrm{U}$ & $\mathrm{U}$ & $\mathrm{U}$ & $\mathrm{U}$ & $\mathrm{U}$ & $\mathrm{U}$ & $\begin{array}{l}\text { Unable to } \\
\text { determine } \\
\text { effectiveness }\end{array}$ \\
\hline \multicolumn{10}{|c|}{ THORACIC RADIOFREQUENCY } \\
\hline $\begin{array}{l}\text { Joo et al, } 2013(120) \\
\text { Quality Scores: } \\
\text { Cochrane }=10 / 12 \\
\text { IPM-QRB }=38 / 48\end{array}$ & 40 & $\begin{array}{l}\text { Radiofrequency } \\
\text { neurotomy }=20 \\
\text { Alcohol injection }=20\end{array}$ & $\begin{array}{l}\text { SI in } \\
\text { both } \\
\text { groups }\end{array}$ & $\begin{array}{l}\text { SI in both } \\
\text { groups }\end{array}$ & $\begin{array}{l}\text { SI in both } \\
\text { groups }\end{array}$ & $\mathrm{P}$ & $\mathrm{P}$ & $\mathrm{P}$ & $\begin{array}{l}\text { Short- and } \\
\text { long-term } \\
\text { effectiveness of } \\
\text { radiofrequency } \\
\text { neurotomy } \\
\text { in alcohol } \\
\text { injection group. }\end{array}$ \\
\hline \multicolumn{10}{|c|}{ THORACIC FACET JOINT NERVE BLOCKS } \\
\hline $\begin{array}{l}\text { Manchikanti et al, } 2012 \\
(84) \\
\text { RA, DB } \\
\text { Quality Scores: } \\
\text { Cochrane = 10/12 } \\
\text { IPM-QRB = 45/48 }\end{array}$ & 100 & $\begin{array}{l}\text { Local anesthetic }=50 \\
\text { Local anesthetic with } \\
\text { steroid }=50\end{array}$ & $\begin{array}{l}79 \% \text { vs } \\
83 \%\end{array}$ & $\begin{array}{l}79 \% \text { vs } \\
81 \%\end{array}$ & $\begin{array}{l}80 \% \text { vs } \\
83 \%\end{array}$ & $\mathrm{P}$ & $\mathrm{P}$ & $\mathrm{P}$ & $\begin{array}{l}\text { Short- and } \\
\text { long-term } \\
\text { effectiveness }\end{array}$ \\
\hline
\end{tabular}

$\mathrm{RA}=$ randomized; $\mathrm{DB}=$ double-blind $\mathrm{P}=$ prospective; $\mathrm{R}=$ retrospective; vs = versus; $P$ = positive

results showed that in the sham control group $58 \%$ of the patients and in the active treatment group $25 \%$ of the patients had their pain come back immediately after the procedure at the 3-month follow-up. Lord et al (128) were obviously unable to avoid this issue of different return of pain in 2 different groups and this is a problem with any of the interventional techniques. In contrast to the criticism of Carragee et al (157), Dreyfuss and Baker (158) supported Lord et al's manuscript (128) for maintaining appropriate blinding of patients based on the fact that it was very difficult to maintain a lack of anesthetic effect and also the numerous difficulties encountered in performing such studies, which is evidenced by the lack of such studies thus far in the cervical spine.

The remaining radiofrequency neurotomy studies were observational in nature. Sapir and Gorup (159), in a 2001 study evaluated the effectiveness of radiofrequency medial branch neurotomy of cervical facet joints after whiplash injury with chronic neck pain in a design which compared litigants to nonlitigants. The inclusion criteria included involvement in a motor vehicle accident at least 20 weeks prior to the study, failure to respond to conservative treatment, and a positive response to controlled, comparative, local anesthetic blocks. They included 50 patients who met inclusion criteria with at least $80 \%$ pain relief from comparative local anesthetic blocks and subsequently underwent radiofrequency neurotomy. However, only 46 of the patients completed the study with 29 in the litigation group (63\%) and 17 in the nonlitigation group (37\%). Subsequent to radiofrequency neurotomy, 21 patients, 14 patients in the litigation group and 7 in the nonlitigation group, experienced recurrence of pain within one year, whereas, 25 patients, 15 in the litigation group and 20 in the nonlitigation group, remained asymptomatic at the end of the one-year follow-up period. They showed that the return of pain, which they defined as $50 \%$ of pain returning, was approximately $8.3 \pm 2.3$ months in the 21 patients whose pain returned within one year. There were no significant differences in relation to the outcomes between the litigant and nonlitigant groups. Overall the authors concluded that cervical radiofrequency neurotomy of facet joints in 
chronic neck pain secondary to whiplash injury was an effective modality independent of litigation.

In another study by MacVicar (160), which was derived from 2 practices in New Zealand, a successful outcome was reported in $74 \%$ and $61 \%$ of patients with long-lasting relief of 17 to 20 months from the first radiofrequency neurotomy, and 15 months for repeat radiofrequency neurotomy. Considering the need for repeat treatments, which were provided appropriately, overall the patients maintained relief for a median duration of 20 to 26 months, with $60 \%$ continuing to have relief with one radiofrequency neurotomy procedure. The authors concluded that radiofrequency neurotomy is an effective technique when performed in a rigorous manner with appropriate selection of patients and consideration of the procedural requirements in chronic neck pain secondary to cervical facet joint involvement temporarily, but completely, relieved of pain, restoring patients to desired activities of daily living. In another study by Speldewinde (161), with 379 procedures, 272 or $72 \%$ of the procedures were considered successful by the patients, irrespective of the region treated. He showed a large effect size with significant improvement. He also showed that repetition of the procedure was highly successful. He concluded that radiofrequency neurotomy of not only the cervical facet joints, but also thoracic and lumbar facets and sacroiliac joints were uniformly successful with $72 \%$ of recipients obtaining an average of $86 \%$ reduction in pain for a period of 12 months. Other studies by McDonald et al (163) and Barnsley et al (164) also demonstrated significant progress on a long-term basis. McDonald et al (163), in an assessment of long-term follow-up of patients, performed cervical radiofrequency neurotomy for chronic neck pain and showed successful results with complete pain relief in $71 \%$ of the patients after an initial procedure; however, the pain returned after 290 days when failures were included. Otherwise, they reported 422 days of relief with all successful cases. The major deficiency was that it included only 28 patients, which appears to have been replicating the results of the randomized controlled trial (128) with very similar outcomes. Barnsley et al (164) also assessed percutaneous radiofrequency neurotomy for chronic neck pain in 35 patients with 47 procedures. The results showed patients receiving 36 procedures achieved $80 \%$ significant pain relief with a mean duration of pain relief of 36 weeks, with repeat procedures usually achieving reproducible pain relief.

For thoracic radiofrequency neurotomy the level of evidence is Level III based on one high quality randomized controlled trial (120).

The level of evidence for facet joint nerve blocks is Level II in the lumbar, cervical, and thoracic regions based on 4 high quality randomized controlled trials $(82-84,117)$ and one moderate to high quality randomized controlled trial (112). Further, there were no trials showing a lack of effectiveness.

Therapeutic facet joint nerve blocks were studied in all 3 regions with 2 high quality randomized controlled trials $(82,117)$ in the lumbosacral region, and one moderate to high quality randomized controlled trial (112). One high quality randomized controlled trial in the thoracic (84) and cervical region (83). Four of the 5 manuscripts were from the same group of authors (82-84,112). In 2 manuscripts (82,83), 120 patients were included in each of the studies of lumbar and cervical facet joint pain $(82,83)$; in studying thoracic facet joint pain (84) 100 patients were included; whereas, in one lumbar trial (112) 73 patients were included. The patients were all drawn from an interventional pain management practice. They had all failed conservative management and were judged to be positive for facet joint pain utilizing controlled comparative local anesthetic blocks with $80 \%$ pain relief as the criterion standard with ability to perform previously painful movements. In each group, an equal number of patients were allocated to receive either local anesthetic alone or local anesthetic with steroid. Outcome parameters included pain relief criteria and disability criteria with follow-ups at 3, 6, 12, 18, and 24 months. Significant pain relief was defined as greater than $50 \%$ relief with significant improvement in functional status of greater than $40 \%$. In the lumbar region (82), the results showed significant pain relief in $85 \%$ of the patients receiving local anesthetic and $90 \%$ of the patients receiving local anesthetic with steroids at the end of the 2 year study period with an average of 5 to 6 total treatments. In the cervical spine (83), $85 \%$ of the patients in the local anesthetic only group and $93 \%$ of the patients in the steroid and local anesthetic group had significant improvement with a total of 5 to 6 procedures over a period of 2 years.

In the thoracic spine, the results were similar to the cervical and lumbar spines with $80 \%$ of the patients in the local anesthetic group and $84 \%$ of the patients in the local anesthetic and steroid group showing significant improvement at the end of 2 years with a total of 5 to 6 procedures. The fourth study, by Civelek et al (117), studied 100 patients with chronic low back pain who 
failed conservative therapy and implemented strict selection criteria even though no diagnostic blocks were utilized. They used lumbosacral facet joint nerve blocks as the control group, whereas, the second group received conventional radiofrequency neurotomy. They followed the patients for 6 months and 12 months. At the end of one-year, $69 \%$ of the patients in the facet joint nerve block group showed significant improvement compared to $90 \%$ in the radiofrequency neurotomy group. Overall it showed the effectiveness of lumbar facet joint nerve blocks even though they were inferior to radiofrequency neurotomy. The final moderate to high quality study by Manchikanti et al (112), included 73 patients and compared a combination of bupivacaine with Sarapin and bupivacaine with Sarapin with the addition of steroids and showed positive results on a long-term basis.

These results are similar to some previously published systematic reviews (55-57); however, there were no other systematic reviews which appropriately studied the role of facet joint nerve blocks.

The evidence for the lumbar intraarticular injection of steroids is Level III, based on 3 high quality randomized controlled trials, showed effectiveness with shortterm follow-up of less than 6 months $(121,123,124)$; however, the results were also opposed by 2 moderate to high quality randomized controlled trials showing a lack of effectiveness $(110,111)$. This level of evidence is similar to other published systematic reviews. In the cervical spine there were 2 randomized controlled trials of intraarticular injections $(119,129)$ yielding evidence of Level III with one trial showing a lack of effectiveness and the second one showing undetermined results. These results are also similar to previous systematic reviews. There were no studies on intraarticular injections in the thoracic spine.

The disadvantages of this systematic review include the lack of metaanalysis; however, there was no clinical homogeneity among the trials. Further, it would be inappropriate to perform a systematic review based on some hypothetical principle if the trials are not clinically homogenous. Consequently, a best evidence synthesis appears to be the most appropriate in this setting. Other disadvantages include the continued paucity of literature about facet joint nerve blocks in all 3 regions and radiofrequency neurotomy in the cervical and thoracic regions as well as intraarticular injections in the cervical and thoracic regions. Future trials must be of appropriate size, draw from a population from practical settings, with a minimum long-term follow-up of one-year. Mul- tiple other issues related to facet joint interventions include placebo response, nocebo response, the role of sham procedures, technical aspects in performing a procedure, and finally the role of local anesthetic alone compared to steroids with sodium chloride solution or steroids with local anesthetic (165-224). Based on the present evidence, there is no additional effectiveness beyond the relief provided by local anesthetic blocks with the addition of steroids, bupivacaine specifically, in facet joint nerve blocks $(82-84,112,152,162,180)$.

The rationale for intraarticular injections comes from steroids being used for treating inflammation. The literature abounds with reports that epidural corticosteroid injections have significant efficacy for their antiinflammatory, immuno-suppressive, anti-edema effects and inhibition of neurotransmission within the $C$ fibers (185-198). The same is supported with facet joint nerve blocks; with long-term symptomatic improvement very similar to the addition of steroids and even better than with steroids $(82-84,112,152,162,180,189,190,225-234)$. The experimental evidence also shows a lack of effectiveness of adding steroids $(189,190)$.

It has been postulated that local anesthetics provide relief by suppressing nociceptive discharge (190), blocking axonal transport $(191,192)$, blocking the sympathetic reflex arc, blocking sensitization $(193,194)$, and by their anti-inflammatory effects (195). Local anesthetics have been reported to have long-term effectiveness following local anesthetic nerve blocks or epidural injections $(82-84,110,112,152,180,206-208,225-234)$.

The lack of placebo in active control trials is a major misunderstanding and a limitation. However, placebo control has been misunderstood in many cases. The reviewers have considered a local anesthetic injection as a placebo control. It is a well known fact that placebo control in any neural blockade is a difficult task. Further, it also adds ethical issues and difficulty with recruitment in the United States. However, multiple investigations performed in interventional pain management with descriptions of placebo control have design flaws $(62,65,180,199-204)$. A solution's effect when injected into a closed space has been inappropriately appraised. Carrette et al $(110,196)$ reported that the response is similar whether an injectate has a sodium chloride solution or a local anesthetic with a steroid. The response to both injections in both the intraarticular and epidural space was low. Thus, their study (110) shows that sodium chloride solution injected into an intraarticular space has similar effects as local anesthetic with a steroid; the conclusion is that intraarticular steroids are 
not an effective therapy. The issue is also exemplified by Birkenmaier et al (205), utilizing either pericapsular injections or medial branch blocks, who then went on to perform cryoneurolysis. Not surprisingly, the results were superior in patients who were diagnosed using medial branch blocks rather than pericapsular injections of local anesthetic. This study was the basis for Chou and Huffman (7) to reject diagnostic lumbar facet joint never blocks as having value. Also, there are reports of different effects from different solutions such as local anesthetic, normal saline, and dextrose; the same is true when a solution is injected into the disc, facet joint, or multifidus muscle (206-213). It has been shown that a small volume of local anesthetic or normal saline abolishes muscle twitch induced by a low current $(0.5$ $\mathrm{mA}$ ) during electrode location (206-209). Further, there is direct evidence for spinal cord involvement in placebo analgesia (210). It also has been shown that epidurally administered sodium chloride solution provides significant improvement in pain and function $(196,214-217)$. Therefore, it can be concluded that local anesthetic's effect on cervical facet joint nerve blocks is not due to the placebo effect, even though some have mistakenly misinterpreted this to be the case $(200,201,217,218)$.

Placebo effects are not expected to be seen in a high proportion of patients, nor are they expected to be long lasting with repeat interventions over a period of 2 years. However, the limitations of the lack of placebo must not be underestimated. If feasible, a placebo-controlled study with appropriate design that includes not injecting the placebo solution over the facet joint nerves, and subsequent results, would be highly valid and provide conclusive knowledge on the issue of placebo-controlled blocks. The issues related to placebo have been discussed extensively in recent years ultimately leading to the opinion that the placebo effect is an inconsistent measure in clinical studies, unless it is designed appropriately $(166,167,169,170,219-224)$.

Another issue is related to the reliability of controlled, comparative local anesthetic blocks, which have been criticized, and their validity as precision diagnostic techniques has been questioned and debated $(7,10,16-$ $19,62,199,235-239)$. The issues related to the accuracy of diagnostic facet joint nerve blocks include the reference standard, prior exposure to opioids, sedation, systemic local anesthetic effect, and non-specific effect resulting in positive results $(7,10,16-19,56,62,209,240-249)$. The validity of controlled facet joint nerve blocks as a gold standard or reference standard in the diagnosis of lumbar facet joint pain has been established $(248,249)$.
A reference standard is established in surgical situations via biopsy or autopsy. However, these are difficult to apply in the diagnosis of chronic spinal pain of facet joint origin. Thus, the long-term or dedicated clinical follow-up of patients appears to be the only solution in establishing a reference standard with controlled facet joint nerve blocks (250). Based on the criterion standard of long-term follow-up, controlled diagnostic lumbar facet joint nerve blocks have been shown to be valid utilizing the criteria of $80 \%$ pain relief and the ability to perform previously painful movements, with a sustained diagnosis of lumbar facet joint pain in at least $89.5 \%$ of the patients at the end of 2-year follow-up (248). However, the diagnosis was sustained in only $51 \%$ of the patients with $50 \%$ relief at the end of 2 years (248). Thus, the controlled diagnostic blocks utilized in this study appear to be reliable.

\subsection{Conclusion}

This systematic review shows Level II evidence for long-term effectiveness of radiofrequency neurotomy in the lumbar and cervical spines, for facet joint nerve blocks in the cervical, thoracic, and lumbar spine, and Level III evidence for thoracic radiofrequency neurotomy, lumbar intraarticular injections and cervical intraarticular injections. This systematic review was performed utilizing strict inclusion criteria and methodological quality assessment criteria. Overall, the results appear to be somewhat superior in patients who receive conventional radiofrequency neurotomy after undergoing controlled diagnostic blocks.

\section{Acknowledgments}

The authors wish to thank Vidyasagar Pampati, MSc, for statistical assistance, and Tom Prigge, MA, Laurie Swick, BS, and Sanjana Pampati, BS, for manuscript review, and Tonie M. Hatton and Diane E. Neihoff, transcriptionists, for their assistance in preparation of this manuscript. We would like to thank the editorial board of Pain Physician for review and criticism in improving the manuscript.

\section{Author Affiliations}

Dr. Manchikanti is Medical Director of the Pain Management Center of Paducah, Paducah, KY, and Clinical Professor, Anesthesiology and Perioperative Medicine, University of Louisville, Louisville, KY.

Dr. Kaye is Professor and Chair, Department of Anesthesia, LSU Health Science Center, New Orleans, LA.

Dr. Boswell is Professor and Chair, Department of 
Anesthesiology and Perioperative Medicine, University of Louisville, Louisville, KY

Dr. Bakshi is President of Manhattan Spine and Pain Medicine, New York, NY.

Dr. Gharibo is Medical Director of Pain Medicine and Associate Professor of Anesthesiology and Orthopedics, Department of Anesthesiology, NYU LangoneHospital for Joint Diseases, NYU School of Medicine, New York, NY.

Dr. Grami is an Interventional Pain Physician at Geisinger Medical Center Interventional Pain Center Woodbine, Danville, PA.

Dr. Grider is Medical Director, UK HealthCare Pain Services, Division Chief, Pain and Regional Anesthesia, and Associate Professor, Department of Anesthesiology, University of Kentucky, Lexington, KY.

Dr. Gupta is a Consultant in Pain Medicine and Anaesthesia, Bradford Teaching Hospital NHS Foundation Trust, Bradford Royal Infirmary, Bradford, United Kingdom.

Dr. Jha is an anesthesiology resident physician at Rush University Medical Center (RUMC), Chicago, IL.

Dr. Mann is Medical Director, Garden State Pain Management, Whiting, NJ.

Dr. Nampiaparampil is Assistant Professor, Rehabilitation Medicine, New York University School of Medicine, New York, NY.

Dr. Sharma is a Consultant in Pain Medicine, Department of Pain Medicine, The Walton Centre for Neurology and Neurosurgery NHS Foundation Trust, Liverpool, and a Fellow of the Faculty of Pain Medicine of the Royal College of Anaesthetists and Treasurer of British Pain Society Interventional Pain Medicine Special Interest Group, United Kingdom.

Dr. Shroyer is an Interventional Pain Physician at Florida Pain Management Associates, Sebastian, FL.
Dr. Singh is Medical Director, Spine Pain Diagnostics Associates, Niagara, WI

Dr. Soin is Medical Director, Ohio Pain Clinic, Centerville, $\mathrm{OH}$.

Dr. Vallejo is Director of Research, Millennium Pain Center, Bloomington, IL; and Adjunct Professor of Biology, Illinois State University, Normal, IL

Dr. Wargo is an Interventional Pain Physician at the McFarland Clinic, Mary Greeley Medical Center, Ames, IA.

Dr. Hirsch is Vice Chief of Interventional Care, Chief of Neurolnterventional Spine, Service Line Chief of Interventional Radiology, Director of Neurolnterventional Services and Neuroendovascular Program, Massachusetts General Hospital; and Associate Professor, Harvard Medical School, Boston, MA.

\section{Conflict of interest:}

Dr. Manchikanti has provided limited consulting services to Semnur Pharmaceuticals, Incorporated, which is developing nonparticulate steroids.

Dr. Kaye is a speaker for Depomed, Inc.

Dr. Gupta has been paid honorarium for presenting at meetings and teaching on the interventional pain medicine cadaver courses and by pharmaceutical companies for presenting to health care professionals. Pharmaceutical companies and companies that manufacture equipments used in pain medicine have supported meetings organized by Dr S Gupta

Dr. Vallejo receives research support from CephaIon/Teva, BioDelivery Sciences International, Inc., Mundipharma Research GmbH \& Co., AstraZeneca, Purdue Pharma, LP, and Theravance, and is a consultant for Nevro and Kimberly-Clark.

Dr. Hirsch is a consultant for Medtronic. 


\section{References}

1. US Burden of Disease Collaborators. The state of US health, 1999-2010: Burden of diseases, injuries, and risk factors. JAMA 2013; 310:591-608.

2. Institute of Medicine (IOM). Relieving Pain in America: A Blueprint for Transforming Prevention, Care, Education, and Research. The National Academies Press, Washington, DC, June 29, 2011.

3. Martin BI, Turner JA, Mirza SK, Lee MJ, Comstock BA, Deyo RA. Trends in health care expenditures, utilization, and health status among US adults with spine problems, 1997-2006. Spine (Phila Pa 1976) 2009; 34:2077-2084.

4. Gaskin DJ, Richard P. The economic costs of pain in the United States. J Pain 2012; 13:715-724.

5. Hoy D, March L, Brooks $P$, Blyth $F$, Woolf A, Bain C, Williams G, Smith E, Vos T, Barendregt J, Murray C, Burstein $\mathrm{R}$, Buchbinder R. The global burden of low back pain: Estimates from the Global Burden of Disease 2010 study. Ann Rheum Dis 2014; 73:968-974.

6. Hoy D, March L, Woolf A, Blyth F, Brooks $P$, Smith E, Vos T, Barendregt J, Blore J, Murray C, Burstein R, Buchbinder R. The global burden of neck pain: Estimates from the global burden of disease 2010 study. Ann Rheum Dis 2014; 73:1309-1315.

7. Chou R, Huffman L. Guideline for the Evaluation and Management of Low Back Pain: Evidence Review. American Pain Society, Glenview, IL, 2009.

8. Manchikanti L, Singh V, Falco FJE, Benyamin RM, Hirsch JA. Epidemiology of low back pain in adults. Neuromodulation 2014; 17:3-10.

9. Manchikanti L, Pampati V, Falco FJE, Hirsch JA. Growth of spinal interventional pain management techniques: Analysis of utilization trends and Medicare expenditures 2000 to 2008 . Spine (Phila Pa 1976) 2013; 38:157-168.

10. Manchikanti L, Abdi S, Atluri S, Benyamin RM, Boswell MV, Buenaventura RM, Bryce DA, Burks PA, Caraway DL, Calodney AK, Cash KA, Christo PJ, Cohen SP, Colson J, Conn A, Cordner HJ, Coubarous S, Datta S, Deer TR, Diwan SA, Falco FJE, Fellows B, Geffert SC, Grider JS, Gupta S, Hameed H, Hameed $M$, Hansen $H$, Helm II S, Janata JW, Justiz R, Kaye $A D$, Lee $M$, Manchikanti KN, McManus CD, Onyewu O, Parr AT, Patel VB, Racz GB, Sehgal N, Sharma $M$, Simopoulos TT, Singh $V$, Smith HS, Snook LT, Swicegood J, Vallejo R, Ward
SP, Wargo BW, Zhu J, Hirsch JA. An update of comprehensive evidence-based guidelines for interventional techniques of chronic spinal pain: Part II: Guidance and recommendations. Pain Physician 2013; 16:S49-S283

11. Rubinstein SM, van Middelkoop M, Assendelft WJ, de Boer MR, van Tulder MW. Spinal manipulative therapy for chronic low-back pain: An update of a Cochrane review. Spine (Phila Pa 1976) 2011; 36:E825-E846.

12. van Middelkoop M, Rubinstein SM, Kuijpers T, Verhagen AP, Ostelo R, Koes BW, van Tulder MW. A systematic review on the effectiveness of physical and rehabilitation interventions for chronic nonspecific low back pain. Eur Spine J 2011; 20:19-39.

13. Manchikanti L, Pampati V, Falco FJE, Hirsch JA. An updated assessment of utilization of interventional pain management techniques in the Medicare population: 2000 - 2013. Pain Physician 2015; 18:E115-E127.

14. Manchikanti L, Pampati V, Singh V, Falco FJE. Assessment of the escalating growth of facet joint interventions in the Medicare population in the United States from 2000 to 2011. Pain Physician 2013; 16:E365-E378.

15. Manchikanti L, Helm II S, Singh V, Hirsch JA. Accountable interventional pain management: A collaboration among practitioners, patients, payers, and government. Pain Physician 2013; 16:E635-E670.

16. Rubinstein SM, van Tulder M. A best-evidence review of diagnostic procedures for neck and low-back pain. Best Pract Res Clin Rheumatol 2008; 22:471-482.

17. Hancock MJ, Maher CG, Latimer J, Spindler MF, McAuley JH, Laslett M, Bogduk N. Systematic review of tests to identify the disc, SIJ or facet joint as the source of low back pain. Eur Spine ] 2007; 16:1539-1550.

18. Bogduk N. On diagnostic blocks for lumbar zygapophysial joint pain. F100o Med Rep 2010; 2:57.

19. Boswell MV, Manchikanti L, Kaye AD, Bakshi S, Gharibo CG, Gupta S, Jha S, Nampiaparampil DE, Simopoulos TT, Hirsch JA. A best-evidence systematic appraisal of the diagnostic accuracy and utility of facet (zygapophysial) joint injections in chronic spinal pain. Pain Physician 2015; 18:E497-533..

20. Kuslich SD, Ulstrom CL, Michael CJ. The tissue origin of low back pain and sciatica: A report of pain response to tissue stimulation during operation on the lumbar spine using local anesthesia. Orthop Clin North Am 1991; 22:181-187.

21. Kalichman L, Li L, Kim DH, Guermazi A, Berkin V, O'Donnell CJ, Hoffmann U, Cole R, Hunter DJ. Facet joint osteoarthritis and low back pain in the community-based population. Spine (Phila Pa 1976) 2008; 33:2560-2565.

22. Eubanks JD, Lee MJ, Cassinelli E, Ahn NU. Prevalence of lumbar facet arthrosis and its relationship to age, sex, and race: An anatomic study of cadaveric specimens. Spine (Phila Pa 1976) 2007; 32:2058-2062.

23. Harris RI, Macnab I. Structural changes in the lumbar intervertebral discs; their relationship to low back pain and sciatica. J Bone Joint Surg Br 1954; 36-B:304-322.

24. Bykowski JL, Wong WH. Role of facet joints in spine pain and image-guided treatment: A review. AJNR Am ] Neuroradiol 2012; 33:1419-1426.

25. Tessitore E, Molliqaj G, Schatlo B, Schaller K. Clinical evaluation and surgical decision making for patients with lumbar discogenic pain and facet syndrome. Eur J Radiol 2015; 84:765-770.

26. Gellhorn AC, Katz JN, Suri P. Osteoarthritis of the spine: The facet joints. Nat Rev Rheumatol 2013; 9:216-224.

27. Igarashi A, Kikuchi S, Konno S, Olmarker K. Inflammatory cytokines released from the facet joint tissue in degenerative lumbar spinal disorders. Spine (Phila Pa 1976) 2004; 29:2091-2095.

28. Schulte TL, Filler TJ, Struwe P, Liem D, Bullman V. Intra-articular meniscoid folds in thoracic zygapophysial joints. Spine (Phila Pa 1976) 2010; 35:E191-E197.

29. Kim JS, Kroin JS, Buvanendran A, Li X, van Wijnen AJ, Tuman KJ, Im HJ. Characterization of a new animal model for evaluation and treatment of back pain due to lumbar facet joint osteoarthritis. Arthritis Rheum 2011; 63:2966-2973.

30. Masini M, Paiva WS, Araujo AS, Jr. Anatomical description of the facet joint innervation and its implication in the treatment of recurrent back pain. J Neurosurg Sci 2005; 49:143-146.

31. Cavanaugh JM, Lu Y, Chen C, Kallakuri $S$. Pain generation in lumbar and cervical facet joints. J Bone Joint Surg Am 2006; 88:63-67.

32. Bogduk N, Wilson AS, Tynan W. The human lumbar dorsal rami. J Anat 1982; 134:383-397. 
33. Bogduk N. The clinical anatomy of the cervical dorsal rami. Spine (Phila Pa 1976) 1982; 7:319-330.

34. Ohtori S, Takahashi K, Chiba T, Yamagata M, Sameda H, Moriya H. Sensory innervation of the cervical facet joints in rats. Spine (Phila Pa 1976) 2001; 26:147-150.

35. Barnsley L, Bogduk N. Medial branch blocks are specific for the diagnosis of cervical zygapophyseal joint pain. Reg Anesth 1993; 18:343-350.

36. Zhang J, Tsuzuki N, Hirabayashi S, Saiki K, Fujita K. Surgical anatomy of the nerves and muscles in the posterior cervical spine. Spine (Phila Pa 1976) 2003; 28:1379-1384.

37. Siegenthaler A, Schliessbach J, Curatolo $\mathrm{M}$, Eichenberger U. Ultrasound anatomy of the nerves supplying the cervical zygapophyseal joints: An exploratory study. Reg Anesth Pain Med 2011; 36:606-610.

38. Kallakuri S, Li Y, Chen C, Cavanaugh JM. Innervation of cervical ventral facet joint capsule: Histological evidence. World J Orthop 2012; 3:10-14.

39. Chua WH, Bogduk N. The surgical anatomy of thoracic facet denervation. Acta Neurochir 1995; 136:140-144.

40. Steinke H, Saito T, Miyaki T, Oi Y, Itoh M, Spanel-Borowski K. Anatomy of the human thoracolumbar Rami dorsales nervi spinalis. Ann Anat 2009; 191:408-416.

41. Sato T, Koizumi M, Kim JH, Kim JH, Wang BJ, Murakami G, Cho BH. Fetal development of deep back muscles in the human thoracic region with a focus on transversospinalis muscles and the medial branch of the spinal nerve posterior ramus. J Anat 2011; 219:756-765.

42. Marks RC. Distribution of pain provoked from lumbar facet joints and related structures during diagnostic spinal infiltration. Pain 1989; 39:37-40.

43. Fukui S, Ohseto K, Shiotani M, Ohno K, Karasawa H, Naganuma Y. Distribution of referred pain from the lumbar zygapophyseal joints and dorsal rami. Clin J Pain 1997; 13:303-307.

44. Hirsch C, Ingelmark BE, Miller M. The anatomical basis for low back pain. Studies on the presence of sensory nerve endings in ligamentous, capsular and intervertebral disc structures in the human lumbar spine. Acta Orthop Scand 1963; 33:1-17.

45. Windsor RE, King FJ, Roman SJ, Tata NS, Cone-Sullivan LA, Thmapi S, Acebey M, Gilhool JJ, Rao R, Sugar R. Electri- cal stimulation induced lumbar medial branch referral patterns. Pain Physician 2002; 5:347-353.

46. Mooney V, Robertson J. The facet syndrome. Clin Orthop Relat Res 1976; 115:149-156.

47. McCall IW, Park WM, O'Brien JP. Induced pain referral from posterior lumbar elements in normal subjects. Spine (Phila Pa 1976) 1979; 4:441-446.

48. Dreyfuss P, Tibiletti C, Dreyer SJ. Thoracic zygapophyseal joint pain patterns: A study in normal volunteers. Spine (Phila Pa 1976) 1994; 19:807-811.

49. Fukui S, Ohseto K, Shiotani M. Patterns of pain induced by distending the thoracic zygapophyseal joints. Reg Anesth 1997; 22:332-336.

50. Dwyer A, Aprill C, Bogduk N. Cervical zygapophyseal joint pain patterns: A study in normal volunteers. Spine (Phila Pa 1976) 1990; 15:453-457.

51. Aprill C, Dwyer A, Bogduk N. The prevalence of cervical zygapophyseal joint pain patterns II: A clinical evaluation. Spine (Phila Pa 1976) 1990; 15:458-461.

52. Fukui S, Ohseto K, Shiotani M, Ohno K, Karasawa H, Naganuma Y, Yuda Y. Referred pain distribution of the cervical zygapophyseal joints and cervical dorsal rami. Pain 1996; 68:79-83.

53. Windsor RE, Nagula D, Storm S. Electrical stimulation induced cervical medial branch referral patterns. Pain Physician 2003; 6:411-418.

54. Bogduk N. International Spinal Injection Society guidelines for the performance of spinal injection procedures. Part 1: Zygapophyseal joint blocks. Clin J Pain 1997; 13:285-302.

55. Falco FJE, Manchikanti L, Datta S, Sehgal N, Geffert S, Onyewu O, Zhu J, Coubarous S, Hameed M, Ward SP, Sharma $M$, Hameed $H$, Singh V, Boswell MV. An update of the effectiveness of therapeutic lumbar facet joint interventions. Pain Physician 2012; 15:E909-E953.

56. Falco FJE, Manchikanti L, Datta S, Wargo BW, Geffert S, Bryce DA, Atluri S, Singh V, Benyamin RM, Sehgal N, Ward S, Helm II S, Gupta S, Boswell MV. Systematic review of therapeutic effectiveness of cervical facet joint interventions: An update. Pain Physician 2012; 15:E839-E868.

57. Manchikanti KN, Atluri S, Singh V, Geffert S, Sehgal N, Falco FJE. An update of evaluation of therapeutic thoracic facet joint interventions. Pain Physician 2012; 15: $\mathrm{E}_{463}-\mathrm{E}_{4} 81$.
58. Leggett LE, Soril LJ, Lorenzetti DL, Noseworthy T, Steadman R, Tiwana S, Clement $F$. Radiofrequency ablation for chronic low back pain: A systematic review of randomized controlled trials. Pain Res Manag 2014; 19:e146-e153.

59. Poetscher AW, Gentil AF, Lenza M, Ferretti $M$. Radiofrequency denervation for facet joint low back pain: A systematic review. Spine (Phila Pa 1976) 2014; 39:E842-E849.

6o. Bogduk N, Dreyfuss P, Govind J. A narrative review of lumbar medial branch neurotomy for the treatment of back pain. Pain Med 2009; 10:1035-1045.

61. Bogduk N. A narrative review of intraarticular corticosteroid injections for low back pain. Pain Med 2005; 6:287-296.

62. Manchikanti L, Datta S, Gupta S, Munglani R, Bryce DA, Ward SP, Benyamin RM, Sharma ML, Helm II S, Fellows B, Hirsch JA. A critical review of the American Pain Society clinical practice guidelines for interventional techniques: Part 2. Therapeutic interventions. Pain Physician 2010; 13:E215-E264.

63. Manchikanti L, Singh V, Derby $R$, Schultz DM, Benyamin RM, Prager JP, Hirsch JA. Reassessment of evidence synthesis of occupational medicine practice guidelines for interventional pain management. Pain Physician 2008; 11:393-482.

64. Niemisto L, Kalso E, Malmivaara A, Seitsalo $S$, Hurri $H$. Radiofrequency denervation for neck and back pain. A systematic review of randomized controlled trials. Cochrane Database Syst Rev 2003; CDoo4058.

65. Manchikanti L, Benyamin RM, Falco FJE, Caraway DL, Datta S, Hirsch JA. Guidelines warfare over interventional techniques: Is there a lack of discourse or straw man? Pain Physician 2012; 15:E1-E26.

66. Chou R, Hashimoto R, Friedly J, Fu Rochelle, Dana T, Sullivan S, Bougatsos C, Jarvik J. Pain Management Injection Therapies for Low Back Pain. Technology Assessment Report ESIBo813. (Prepared by the Pacific Northwest Evidence-based Practice Center under Contract No. HHSA 290-2012-00014I.) Rockville, MD: Agency for Healthcare Research and Quality; March 20, 2015.

www.cms.gov/Medicare/Coverage/DeterminationProcess/Downloads/idg8TA. pdf

67. Health Technology Assessment, Washington State Health Care Authority. Spi- 
nal Injections, Updated Final Evidence Report. Spectrum Research, Inc., March 10, 2011.

68. Database of Abstracts of Reviews of Effects (DARE): Quality-assessed Reviews. York (UK): Centre for Reviews and Dissemination. An update of the effectiveness of therapeutic lumbar facet joint interventions. 2012 (cited 2013 Feb 21).

69. Liberati A, Altman DG, Tetzlaff J, Mulrow C, Gøtzsche PC, loannidis JP, Clarke M, Devereaux PJ, Kleijnen J, Moher D. The PRISMA statement for reporting systematic reviews and meta-analyses of studies that evaluate health care interventions: Explanation and elaboration. Ann Intern Med 2009; 151:W65-W94.

70. Furlan AD, Pennick V, Bombardier C, van Tulder M; Editorial Board, Cochrane Back Review Group. 2009 updated method guidelines for systematic reviews in the Cochrane Back Review Group. Spine (Phila Pa 1976) 2009; 34:1929-1941.

71. Manchikanti L, Falco FJE, Singh V, Benyamin RM, Racz GB, Helm II S, Caraway DL, Calodney AK, Snook LT, Smith HS, Gupta S, Ward SP, Grider JS, Hirsch JA. An update of comprehensive evidence-based guidelines for interventional techniques of chronic spinal pain. Part I: Introduction and general considerations. Pain Physician 2013; 16:S1-S48.

72. Manchikanti L, Hirsch JA, Cohen SP, Heavner JE, Falco FJE, Diwan S, Boswell MV, Candido KD, Onyewu O, Zhu J, Sehgal N, Kaye AD, Benyamin RM, Helm II S, Singh V, Datta S, Abdi S, Christo PJ, Hameed H, Hameed M, Vallejo R, Pampati V, Racz GB, Raj PP. Assessment of methodologic quality of randomized trials of interventional techniques: Development of an interventional pain management specific instrument. Pain Physician 2014; 17:E263-E290.

73. Manchikanti L, Hirsch JA, Heavner JE, Cohen SP, Benyamin RM, Sehgal N, Falco FJE, Vallejo R, Onyewu O, Zhu J, Kaye $A D$, Boswell MV, Helm II S, Candido KD, Diwan S, Simopoulos TT, Singh V, Pampati V, Racz GB, Raj PP. Development of an interventional pain management specific instrument for methodologic quality assessment of nonrandomized studies of interventional techniques. Pain Physician 2014; 17:E291-E317.

74. Farrar JT. What is clinically meaningful: Outcome measures in pain clinical trials. Clin J Pain 2000; 16:S106-S112.

75. Salaffi F, Stancati A, Silvestri CA, Ciapetti A, Grassi W. Minimal clinically important changes in chronic musculoskeletal pain intensity measured on a numerical rating scale. Eur J Pain 2004; 8:283-291.

76. Hagg O, Fritzell P, Nordwall A. The clinical importance of changes in outcome scores after treatment for chronic low back pain. Eur Spine J 2003; 12:12-20.

77. Carragee EJ. The rise and fall of the "minimum clinically important difference." Spine J 2010; 10:283-284.

78. Gatchel RJ, Mayer TG, Choi Y, Chou R. Validation of a consensus-based minimal clinically important difference (MCID) threshold using an objective functional external anchor. Spine ] 2013; 13:889-893.

79. Parker SL, Adogwa O, Mendenhall SK Shau DN, Anderson WN, Cheng JS, Devin CJ, McGirt MJ. Determination of minimum clinically important difference $(M C I D)$ in pain, disability, and quality of life after revision fusion for symptomatic pseudoarthrosis. Spine J 2012; 12:1122-1128.

8o. Copay AG, Subach BR, Glassman SD, Polly DW Jr, Schuler TC. Understanding the minimum clinically important difference: A review of concepts and methods. Spine ] 2007; 7:541-546.

81. Rouquette $A$, Blanchin $M$, Sébille $V$, Guillemin F, Côté SM, Falissard B, Hardouin JB. The minimal clinically important difference determined using item response theory models: An attempt to solve the issue of the association with baseline score. J Clin Epidemiol 2014; 67:433-440.

82. Manchikanti L, Singh V, Falco FJE, Cash KA, Pampati V. Evaluation of lumbar facet joint nerve blocks in managing chronic low back pain: A randomized, double-blind, controlled trial with a 2-year follow-up. Int J Med Sci 2010; 7:124-135.

83. Manchikanti L, Singh V, Falco FJE, Cash KA, Fellows B. Comparative outcomes of a 2-year follow-up of cervical medial branch blocks in management of chronic neck pain: A randomized, double-blind controlled trial. Pain Physician 2010; 13:437-450.

84. Manchikanti L, Singh V, Falco FJE, Cash KA, Pampati V, Fellows B. The role of thoracic medial branch blocks in managing chronic mid and upper back pain: A randomized, double-blind, activecontrol trial with a 2-year follow-up. Anesthesiol Res Pract 2012; 2012:585806.

85. Manchikanti L, Cash KA, McManus CD, Pampati V. Assessment of effectiveness of percutaneous adhesiolysis in managing chronic low back pain secondary to lumbar central spinal canal stenosis. Int J Med Sci 2013; 10:50-59.

86. Manchikanti L, Singh V, Cash KA, Pampati $V$, Datta $S$. Assessment of effectiveness of percutaneous adhesiolysis and caudal epidural injections in managing lumbar post surgery syndrome: A 2-year follow-up of randomized, controlled trial. J Pain Res 2012; 5:597-608.

87. Manchikanti L, Singh V, Cash KA, Pampati V, Damron KS, Boswell MV. Effect of fluoroscopically guided caudal epidural steroid or local anesthetic injections in the treatment of lumbar disc herniation and radiculitis: A randomized, controlled, double blind trial with a two-year follow-up. Pain Physician 2012; 15:273-286.

88. Manchikanti L, Singh V, Cash KA, Pampati V, Datta S. Fluoroscopic caudal epidural injections in managing post lumbar surgery syndrome: Two-year results of a randomized, double-blind, active-control trial. Int J Med Sci 2012; 9:582-591.

89. Manchikanti L, Cash KA, McManus CD, Pampati V, Fellows B. Results of 2-year follow-up of a randomized, doubleblind, controlled trial of fluoroscopic caudal epidural injections in central spinal stenosis. Pain Physician 2012; 15:371-384.

90. Manchikanti L, Singh V, Cash KA, Pampati V, Falco FJE. A randomized, doubleblind, active-control trial of the effectiveness of lumbar interlaminar epidural injections in disc herniation. Pain Physician 2014; 17:E61-E74.

91. Manchikanti L, Cash KA, McManus CD, Pampati $\mathrm{V}$, Benyamin RM. A randomized, double-blind, active-controlled trial of fluoroscopic lumbar interlaminar epidural injections in chronic axial or discogenic low back pain: Results of a 2-year follow-up. Pain Physician 2013; 16:E491-E504.

92. Manchikanti L, Cash KA, McManus CD, Damron KS, Pampati V, Falco FJE. A randomized, double-blind controlled trial of lumbar interlaminar epidural injections in central spinal stenosis: 2-year follow-up. Pain Physician 2015; 18:79-92.

93. Manchikanti L, Cash KA, Pampati V, Falco FJE. Transforaminal epidural injections in chronic lumbar disc herniation: A randomized, double-blind, active-control trial. Pain Physician 2014; 17:E489-E501.

94. Manchikanti L, Cash KA, Pampati V, Malla Y. Two-year follow-up results of fluoroscopic cervical epidural injections 
in chronic axial or discogenic neck pain: A randomized, double-blind, controlled trial. Int J Med Sci 2014; 11:309-320.

95. Manchikanti L, Cash KA, Pampati V, Wargo BW, Malla Y. A randomized, double-blind, active control trial of fluoroscopic cervical interlaminar epidural in jections in chronic pain of cervical disc herniation: Results of a 2-year follow-up. Pain Physician 2013; 16:465-478.

96. Manchikanti L, Malla Y, Cash KA, McManus CD, Pampati V. Fluoroscopic epidural injections in cervical spinal stenosis: Preliminary results of a randomized, double-blind, active control trial. Pain Physician 2012; 15:E59-E70.

97. Manchikanti L, Malla Y, Cash KA, McManus CD, Pampati V. Fluoroscopic cervical interlaminar epidural injections in managing chronic pain of cervical post-surgery syndrome: Preliminary results of a randomized, double-blind active control trial. Pain Physician 2012; 15:13-26.

98. Lohr KN, Carey TS. Assessing 'best evidence': Issues in grading the quality of studies for systematic reviews.] Comm J Qual Improv 1999; 25:470-479.

99. Clarke M, Oxman AD. Cochrane Reviewer's Handbook 4.0. The Cochrane Collaboration, 1999.

100. National Health and Medical Research Council (NHMRC). How to Use the Evidence: Assessment and Application of Scientific Evidence. NHMRC, Canberra, Australia, 2000.

101. Staal JB, de Bie RA, de Vet HC, Hildebrandt J, Nelemans $P$. Injection therapy for subacute and chronic low back pain: An updated Cochrane review. Spine (Phila Pa 1976) 2009; 34:49-59.

102. Harris RP, Helfand M, Woolf SH, Lohr KN, Mulrow CD, Teutsch SM, Atkins D; Methods Work Group, Third US Preventive Services Task Force. Current methods of the US Preventive Services Task Force. Am J Prevent Med 2001; 20:21-35.

103. Guyatt GH, Cook DJ, Sackett DL, Eckman M, Pauker S. Grades of recommendation for antithrombotic agents. Chest 1998; 114:441S-444S.

104. Gross PA, Barrett TL, Dellinger EP, Krause PJ, Martone WJ, McGowan JE Jr, Sweet RL, Wenzel RP. Purpose of quality standards for infectious diseases. Infectious Diseases Society of America. Clin Infect Dis 1994; 18:421.

105. Manchikanti L, Falco FJE, Benyamin RM, Kaye AD, Boswell MV, Hirsch JA. A modified approach to grading of evidence. Pain Physician 2014; 17:E319-E325.
106. Jeong SY, Kim JS, Choi WS, Hur JW, Ryu KS. The effectiveness of endoscopic radiofrequency denervation of medial branch for treatment of chronic low back pain. J Korean Neurosurg Soc 2014; 56:338-343.

107. Li ZZ, Hou SX, Shang WL, Song KR, Wu WW. Evaluation of endoscopic dorsal ramus rhizotomy in managing facetogenic chronic low back pain. Clin Neurol Neurosurg 2014; 126:11-17.

108. Cohen SP, Williams KA, Kurihara C, Nguyen C, Shields C, Kim P, Griffith SR, Larkin TM, Crooks M, Williams N, Morlando B, Strassels SA. Multicenter, randomized, comparative cost-effectiveness study comparing 0,1 , and 2 diagnostic medial branch (facet joint nerve) block treatment paradigms before lumbar facet radiofrequency denervation. Anesthesiology 2010; 113:395-405.

109. Manchikanti L, Singh V, Falco FJ, Cash KA, Pampati V. Lumbar facet joint nerve blocks in managing chronic facet joint pain: One-year follow-up of a randomized, double-blind controlled trial: Clinical Trial NCToo355914. Pain Physician 2008; 11:121-132.

110. Carette S, Marcoux S, Truchon R, Grondin C, Gagnon J, Allard Y, Latulippe M. A controlled trial of corticosteroid injections into facet joints for chronic low back pain. N Engl J Med 1991; 325:1002-1007.

111. Fuchs S, Erbe T, Fischer HL, Tibesku CO. Intraarticular hyaluronic acid versus glucocorticoid injections for nonradicular pain in the lumbar spine. J Vasc Interv Radiol 2005; 16:1493-1498.

112. Manchikanti L, Pampati V, Bakhit C, Rivera J, Beyer C, Damron K, Barnhill R. Effectiveness of lumbar facet joint nerve blocks in chronic low back pain: A randomized clinical trial. Pain Physician 2001; 4:101-117.

113. Nath S, Nath CA, Pettersson K. Percutaneous lumbar zygapophysial (facet) joint neurotomy using radiofrequency current, in the management of chronic low back pain: A randomized doubleblind trial. Spine (Phila Pa 1976) 2008; 33:1291-1298.

114. van Wijk RM, Geurts JW, Wynne HJ, Hammink E, Buskens E, Lousberg R, Knape JT, Groen GJ. Radiofrequency denervation of lumbar facet joints in the treatment of chronic low back pain: A randomized, double-blind, sham lesion-controlled trial. Clin J Pain 2005; 21:335-344.

115. van Kleef M, Barendse GAM, Kessels A,
Voets HM, Weber WE, de Lange S. Randomized trial of radiofrequency lumbar facet denervation for chronic low back pain. Spine (Phila Pa 1976) 1999; 24:1937-1942.

116. Tekin I, Mirzai H, Ok G, Erbuyun K, Vatansever D. A comparison of conventional and pulsed radiofrequency denervation in the treatment of chronic facet joint pain. Clin J Pain 2007; 23:524-529.

117. Civelek E, Cansever T, Kabatas S, Kircelli A, Yilmaz C, Musluman M, Ofluoglu D, Caner $\mathrm{H}$. Comparison of effectiveness of facet joint injection and radiofrequency denervation in chronic low back pain. Turk Neurosurg 2012; 22:200-206.

118. Dobrogowski J, Wrzosek A, Wordliczek J. Radiofrequency denervation with or without addition of pentoxifylline or methylprednisolone for chronic lumbar zygapophysial joint pain. Pharmacol Rep 2005; 57:475-480.

119. Barnsley L, Lord SM, Wallis BJ, Bogduk N. Lack of effect of intra-articular corticosteroids for chronic pain in the cervical zygapophyseal joints. N Engl J Med 1994; 330:1047-1050.

120. Joo YC, Park JY, Kim KH. Comparison of alcohol ablation with repeated thermal radiofrequency ablation in medial branch neurotomy for the treatment of recurrent thoracolumbar facet joint pain. J Anesth 2013; 27:390-395.

121. Ribeiro LH, Furtado RN, Konai MS, Andreo AB, Rosenfeld A, Natour J. Effect of facet joint injection versus systemic steroids in low back pain: A randomized controlled trial. Spine (Phila Pa 1976) 2013; 38:1995-2002.

122. Moon JY, Lee PB, Kim YC, Choi SP, Sim WS. An alternative distal approach for the lumbar medial branch radiofrequency denervation: A prospective randomized comparative study. Anesth Analg 2013; 116:1133-1140.

123. Lakemeier S, LInd M, Schultz W, FuchsWinkelmann S, Timmesfeld N, Foelsch C, Peterlein CD. A comparison of intraarticular lumbar facet joint steroid injections and lumbar facet joint radiofrequency denervation in the treatment of low back pain: A randomized, controlled, double-blind trial. Anesth Analg 2013; 117:228-235.

124. Yun DH, Kim HS, Yoo SD, Kim DH, Chon JM, Choi SH, Hwang DG, Jung PK. Efficacy of ultrasonography-guided injections in patients with facet syn-

drome of the low lumbar spine. Ann Rehabil Med 2012; 36:66-71. 
125. Obernauer J, Galiano K, Gruber H, Bale R, Obwegeser AA, Schatzer R, Loizides A. Ultrasound-guided versus computed tomography-controlled facet joint injections in the middle and lower cervical spine: A prospective randomized clinica

trial. Med Ultrason 2013; 15:10-15.

126. Wen CB, Li YZ, Sun L, Xiao H, Yang BX, Song L, Liu $\mathrm{H}$. A clinical trial of ultrasound-guided facet joint block in the lumbar spine to treat facet joint related

low back pain. Sichuan Da Xue Xue Bao Yi Xue Ban 2014; 45:712-716.

127. Klessinger S. Zygapophysial joint pain in post lumbar surgery syndrome. The efficacy of medial branch blocks and radiofrequency neurotomy. Pain Med 2013; 14:374-377.

128. Lord S, Barnsley L, Wallis B, McDonald $G$, Bogduk N. Percutaneous radio-frequency neurotomy for chronic cervical zygapophyseal-joint pain. $N$ Engl J Med 1996; 335:1721-1726.

129. Park SC, Kim KH. Effect of adding cervical facet joint injections in a multimodal treatment program for long-standing cervical myofascial pain syndrome with referral pain patterns of cervical facet joint syndrome. J Anesth 2012; 26:738-745.

130. Manchikanti L, Singh V, Falco FJE, Cash KA, Pampati V, Fellows B. Comparative effectiveness of a one-year follow-up of thoracic medial branch blocks in management of chronic thoracic pain: A randomized, double-blind active controlled trial. Pain Physician 2010; 13:535-548.

131. Lilius G, Laasonen EM, Myllynen P, Harilainen A, Gronlund G. Lumbar facet joint syndrome. A randomised clinical trial. J Bone Joint Surg $\mathrm{Br} 1989$; 71:681-684.

132. Marks RC, Houston T, Thulbourne T. Facet joint injection and facet nerve block: A randomised comparison in 86 patients with chronic low back pain. Pain 1992; 49:325-328.

133. Nash TP. Facet joints. Intra-articular steroids or nerve blocks? Pain Clinic 1990; 3:77-82.

134. Leclaire R, Fortin L, Lambert $R$, Bergeron YM, Rossignol M. Radiofrequency facet joint denervation in the treatment of low back pain: A placebocontrolled clinical trial to assess efficacy. Spine (Phila Pa 1976) 2001; 26:1411-1416.

135. Gallagher J, Petriccione Di Vadi PL, Wedley JR, Hamann W, Ryan P, Chikanza I, Kirkham B, Price R, Watson MS, Gra- hame R, Wood S. Radiofrequency facet joint denervation in the treatment of low back pain: A prospective controlled double-blind study to assess its efficacy. Pain Clinic 1994; 7:193-198.

136. Kroll H, Kim D, Danic $M$, Sankey $S$, Gariwala M, Brown M. A randomized, double-blind, prospective study comparing the efficacy of continuous versus pulsed radiofrequency in the treatment of lumbar facet syndrome. J Clin Anesth 2008; 20:534-537.

137. Ackerman WE 3rd, Ahmad M. Pain relief with intraarticular or medial branch nerve blocks in patients with positive lumbar facet joint SPECT imaging: A 12week outcome study. South Med J 2008; 101:931-934.

138. Andres RH, Graupner $\mathrm{T}$, Bärlocher $\mathrm{CB}$, Augsburger A, Fandino J. Laser-guided lumbar medial branch kryorhizotomy. J Neurosurg Spine 2010; 13:341-345.

139. MacVicar J, Borowczyk JM, MacVicar AM, Loughnan BM, Bogduk N. Lumbar medial branch radiofrequency neurotomy in New Zealand. Pain Med 2013; 14:639-645.

140. Kader D, Radha S, Smith F, Wardlaw D, Scott N, Rege A, Pope M. Evaluation of perifacet injections and paraspinal muscle rehabilitation in treatment of low back pain. A randomised controlled trial. Ortop Traumatol Rehabil 2012; 14:251-259.

141. Tzaan W, Tasker R. Percutaneous radiofrequency facet rhizotomy-experience with 118 procedures and reappraisal of its value. Can J Neurol Sci 2000; 27:125-130.

142 Stolker RJ, Vervest AC, Groen GJ. Parameters in electrode positioning in thoracic percutaneous facet denervation: An anatomical study. Acta Neurochir 1994; 128:32-39.

143. Stolker RJ, Vervest AC, Groen GJ. The treatment of chronic thoracic segmental pain by radiofrequency percutaneous partial rhizotomy. J Neurosurg 1994; 80:986-992.

144. Chua NH, Vissers KC, Sluijter ME. Pulsed radiofrequency treatment in interventional pain management: Mechanisms and potential indications - a review. Acta Neurochir (Wien) 2011; 153:763-771.

145. Golovac S. Radiofrequency neurolysis. Neuroimaging Clin N Am 2010; 20:203-214.

146. Mitra R, Do H, Alamin T, Cheng I. Facet pain in thoracic compression fractures. Pain Med 2010; 11:1674-1677.
147. Haufe SM, Mork AR. Endoscopic facet debridement for the treatment of facet arthritic - pain - A novel new technique. Int J Med Sci 2010; 7:120-123.

148. Slappendel R, Crul BJ, Braak GJ, Geurts JW, Booij LH, Voerman VF, de Boo T. The efficacy of radiofrequency lesioning of the cervical spinal dorsal root ganglion in a double blinded randomized study: No difference between $40^{\circ} \mathrm{C}$ and $67^{\circ} \mathrm{C}$ treatments. Pain 1997; 73:159-163.

149. Haspeslagh SR, Van Suijlekom HA, Lame IE, Kessels AG, van Kleef M, Weber WE. Randomised controlled trial of cervical radiofrequency lesions as a treatment for cervicogenic headache: ISRCTNo7444684. BMC Anesthesiol 2006; 6:1.

150. Wallis B, Lord S, Bogduk N. Resolution of psychological distress of whiplash patients following treatment by radiofrequency neurotomy: A randomized, double-blind, placebo-controlled trial. Pain 1997; 73:15-22.

151. Schaerer JP. Radiofrequency facet rhizotomy in the treatment of chronic neck and low back pain. Int Surg 1978; 63:53-59.

152. Manchikanti L, Manchikanti K, Damron $\mathrm{K}$, Pampati V. Effectiveness of cervical medial branch blocks in chronic neck pain: A prospective outcome study. Pain Physician 2004; 7:195-201.

153. Roy C, Chatterjee N, Ganguly S, Sengupta R. Efficacy of combined treatment with medial branch radiofrequency neurotomy and steroid block in lumbar facet joint arthropathy. J Vasc Interv Radiol 2012; 23:1659-1664.

154. Mehnert MJ, Freedman MK. Update on the role of z-joint injection and radiofrequency neurotomy for cervicogenic headache. PM R 2013; 5:221-227.

155. Kim D. Bipolar intra-articular radiofrequency thermocoagulation of the thoracic facet joints: A case series of a new technique. Korean J Pain 2014; 27:43-48.

156. Saltychev M, Laimi K. Re: Poetscher AW, Gentil AF, Lenza M, et al. Radiofrequency denervation for facet joint low back pain: A systematic review. Spine (Phila Pa 1976) 2014;39:E842-9. Spine (Phila Pa 1976) 2014; 39:E1498-E1500.

157. Carragee EJ, Hurwitz EL, Cheng I, Carroll LJ, Nordin M, Guzman J, Peloso P, Holm LW, Côté P, Hogg-Johnson S, van der Velde G, Cassidy JD, Haldeman S; Bone and Joint Decade 2000-2010 Task Force on Neck Pain and Its Associated Disorders. Treatment of neck pain: In- 
jections and surgical interventions: Results of the Bone and Joint Decade 2000-2010 Task Force on Neck Pain and Its Associated Disorders. Spine (Phila Pa 1976) 2008; 33:S153-S169.

158. Dreyfuss P, Baker R. In response to treatment of neck pain. Eur Spine J 2008; 17:1270-1272; author reply 1273-1275.

159. Sapir DA, Gorup JM. Radiofrequency medial branch neurotomy in litigant and non-litigant patients with cervical whiplash. Spine (Phila Pa 1976) 2001; 26:E268-E273.

160. MacVicar J, Borowczyk JM, MacVicar AM, Loughnan BM, Bogduk N. Cervical medial branch radiofrequency neurotomy in New Zealand. Pain Med 2012; 13:647-654.

161. Speldewinde GC. Outcomes of percutaneous zygapophysial and sacroiliac joint neurotomy in a community setting. Pain Med 2011; 12:209-218.

162. Manchikanti L, Manchikanti KN, Manchukonda R, Pampati V, Cash KA. Evaluation of therapeutic thoracic medial branch block effectiveness in chronic thoracic pain: A prospective outcome study with minimum 1-year follow up. Pain Physician 2006; 9:97-105.

163. McDonald G, Lord S, Bogduk N. Longterm follow-up of patients treated with cervical radiofrequency neurotomy for chronic spinal pain. Neurosurgery 1999; 45:61-67.

164. Barnsley L. Percutaneous radiofrequency neurotomy for chronic neck pain: Outcomes in a series of consecutive patients. Pain Med 2005; 6:282-286.

165. Leclaire R, Fortin L, Bergeron Y, Lambert R, Rissignol $M$. In response to: Radiofrequency neurotomy for chronic lumbar facet pain - interpreting the evidence. Pain Pract 2010; 10:261-262.

166. Manchikanti L, Pampati V, Damron KS. The role of placebo and nocebo effects of perioperative administration of sedatives and opioids in interventional pain management. Pain Physician 2005; 8:349-355.

167. Manchikanti L, Giordano J, Fellows B, Hirsch JA. Placebo and nocebo in interventional pain management: $A$ friend or a foe - or simply foes? Pain Physician 2011; 14:E157-E175

168. Wells RE, Kaptchuk TJ. To tell the truth, the whole truth, may do patients harm: The problem of the nocebo effect for informed consent. Am ] Bioeth 2012; 12:22-29.

169. Häuser W, Bartram C, Bartram-Wunn
E, Tölle T. Adverse events attributable to nocebo in randomized controlled drug trials in fibromyalgia syndrome and painful diabetic peripheral neuropathy: Systematic review. Clin J Pain 2012; 28:437-451.

170. Lyby PS, Forsberg JT, Asli O, Flaten MA. Induced fear reduces the effectiveness of a placebo intervention on pain. Pain 2012; 153:1114-1121.

171. Hróbjartsson A, Gøtzsche PC. Placebo interventions for all clinical conditions. Cochrane Database Syst Rev 2010; 1:CDoo3974.

172. Bingel U; Placebo Competence Team. Avoiding nocebo effects to optimize treatment outcome. JAMA 2014; 312:693-694.

173. Colloca L, Finniss D. Nocebo effects, patient-clinician communication, and therapeutic outcomes. JAMA 2012; 307:567-568.

174. Howick J, Bishop FL, Heneghan, Wolstenholme J, Stevens S, Hobbs FDR, Lewith G. Placebo use in the United Kingdom: Results from a national survey of primary care practitioners. PLoS One 2013; 8:e58247.

175. Howick J, Friedemann C, Tsakok M, Watson R, Tsakok T, Thomas J, Perera R, Fleming $S$, Heneghan $C$. Are treatments more effective than placebos? A systematic review and meta-analysis. PLoS One 2013; 8:e62599.

176. Kaptchuk TJ, Friedlander E, Kelley JM, Sanchez MN, Kokkotou E, Singer JP, Kowalczykowski M, Miller FG, Kirsch I, Lembo AJ. Placebos without deception: A Randomized controlled trial in irritable bowel syndrome. PLoS One 2010; 5 : e15591.

177. Petersen GL, Finnerup NB, Colloca L, Amanzio M, Price DD, Jensen TS, Vase $L$. The magnitude of nocebo effects in pain: A meta-analysis. Pain 2014; 155:1426-1434.

178. Ghai B, Kumar K, Bansal D, Dhatt SS Kanukula R, Batra YK. Effectiveness of parasagittal interlaminar epidural local anesthetic with or without steroid in chronic lumbosacral pain: A randomized, double-blind clinical trial. Pain Physician 2015; 18:237-248.

179. Ghai B, Bansal D, Kay JP, Vadaje KS, Wig J. Transforaminal versus parasagittal interlaminar epidural steroid injection in low back pain with radicular pain: A randomized, double-blind, active-control trial. Pain Physician 2015; 17:277-290.

180. Manchikanti L, Nampiaparampil DE, Manchikanti KN, Falco FJE, Singh V, Be- nyamin RM, Kaye AD, Sehgal N, Soin A, Simopoulos TT, Bakshi S, Gharibo CG, Gilligan CJ, Hirsch JA. Comparison of the efficacy of saline, local anesthetics, and steroids in epidural and facet joint injections for the management of spinal pain: A systematic review of randomized controlled trials. Surg Neurol Int 2015; 6:S194-S235.

181. Nagar VR, Birthi P, Grider JS, Asopa A. Systematic review of radiofrequency ablation and pulsed radiofrequency for management of cervicogenic headache. Pain Physician 2015; 18:109-130.

182. Manchikanti L, Kaye AD, Manchikanti KN, Boswell MV, Pampati V, Hirsch JA. Efficacy of epidural injections in the treatment of lumbar central spinal stenosis: A systematic review. Anesth Pain Med 2015; 5:e23139.

183. Manchikanti L, Benyamin RM, Falco FJ, Kaye AD, Hirsch JA. Do epidural injections provide short- and long-term relief for lumbar disc herniation? A systematic review. Clin Orthop Relat Res 2015; 473:1940-1956.

184. Manchikanti L, Nampiaparampil DE, Candido KD, Bakshi S, Grider JS, Falco FJE, Sehgal N, Hirsch JA. Do cervical epidural injections provide long-term relief in neck and upper extremity pain? A systematic review. Pain Physician 2015; 18:39-60.

185. Lee HM, Weinstein JN, Meller ST, Hayashi N, Spratt KF, Gebhart GF. The role of steroids and their effects on phospholipase A2. An animal model of radiculopathy. Spine (Phila Pa 1976) 1998; 23:1191-1196.

186. Johansson A, Hao J, Sjölund B. Local corticosteroid application blocks transmission in normal nociceptive C-fibres. Acta Anaesthesiol Scand 1990; 34:335-338.

187. Pasqualucci A, Varrassi G, Braschi A, Peduto VA, Brunelli A, Marinangeli $F$, Gori F, Colò F, Paladín A, Mojoli F. Epidural local anesthetic plus corticosteroid for the treatment of cervical brachial radicular pain: Single injection verus continuous infusion. Clin J Pain 2007; 23:551-557.

188. Sato C, Sakai A, Ikeda Y, Suzuki H, Sakamoto $A$. The prolonged analgesic effect of epidural ropivacaine in a rat model of neuropathic pain. Anesth Analg 2008; 106:313-320.

189. Tachihara H, Sekiguchi M, Kikuchi S, Konno S. Do corticosteroids produce additional benefit in nerve root infiltration for lumbar disc herniation. Spine (Phila Pa 1976) 2008; 33:743-747. 
190. Arner S, Lindblom U, Meyerson BA, Molander $C$. Prolonged relief of neuralgia after regional anesthetic block. A call for further experimental and systematic clinical studies. Pain 1990; 43:287-297.

191. Lavoie PA, Khazen T, Filion PR. Mechanisms of the inhibition of fast axonal transport by local anesthetics. Neuropharmacology 1989; 28:175-181.

192. Bisby MA. Inhibition of axonal transport in nerves chronically treated with local anesthetics. Exp Neurol 1975; 47:481-489.

193. Katz WA, Rothenberg R. The nature of pain: Pathophysiology. J Clin Rheumatol 2005; 11:S11-15.

194. Melzack R, Coderre TJ, Katz J, Vaccarino AL. Central neuroplasticity and pathological pain. Ann N Y Acad Sci 2001; 933:157-174.

195. Cassuto J, Sinclair R, Bonderovic M. Anti-inflammatory properties of local anesthetics and their present and potential clinical implications. Acta Anaesthesiol Scand 2006; 50:265-282.

196. Carette S, Leclaire R, Marcoux S, Morin F, Blaise GA, St-Pierre A, Truchon R, Parent $F$, Levesque J, Bergeron $V$, Montminy $P$, Blanchette $C$. Epidural corticosteroid injections for sciatica due to herniated nucleus pulposus. $N$ Engl J Med 1997; 336:1634-1640.

197. Karppinen J, Malmivaara A, Kurunlahti $M$, Kyllönen E, Pienimäki T, Nieminen P, Ohinmaa A, Tervonen O, Vanharanta $H$. Periradicular infiltration for sciatica: A randomized controlled trial. Spine ( $\mathrm{Ph}$ ila Pa 1976) 2001; 26:1059-1067.

198. Hayashi N, Weinstein JN, Meller ST, Lee HM, Spratt KF, Gebhart GF. The effect of epidural injection of betamethasone or bupivacaine in a rat model of lumbar radiculopathy. Spine (Phila Pa 1976) 1998 23:877-885.

199. Manchikanti L, Datta S, Derby R, Wolfer LR, Benyamin RM, Hirsch JA. A critical review of the American Pain Society clinical practice guidelines for interventional techniques: Part 1. Diagnostic interventions. Pain Physician 2010; 13:E141-E174.

200. Manchikanti L, Shah RV, Datta S, Singh V. Critical evaluation of interventional pain management literature provides inaccurate conclusions. Spine J 2009; 9:706-708.

201. Smuck M, Levin JH: RE: Manchikanti L, Singh V, Falco FJE, Cash KA, Fellows B. Cervical medial branch blocks for chronic cervical facet joint pain: A randomized double-blind, controlled trial with one-year follow-up. Spine (Phila Pa
1976) 2008; 33:1813-20. Spine (Phila Pa 1976) 2009; 34:1116.

202. Chou R, Atlas SJ, Stanos SP, Rosenquist RW. Nonsurgical interventional therapies for low back pain: A review of the evidence for an American Pain Society clinical practice guideline. Spine (Phila Pa 1976) 2009; 34:1078-1093.

203. Manchikanti L, Falco FJE, Boswell MV, Hirsch JA. Facts, fallacies, and politics of comparative effectiveness research: Part 2. Implications for interventional pain management. Pain Physician 2010; 13:E55-E79.

204. Chou R, Atlas SJ, Loeser JD, Rosenquist RW, Stanos SP. Guideline warfare over interventional therapies for low back pain: Can we raise the level of discourse? J Pain 2011; 12:833-839.

205. Birkenmaier C, Veihelmann A, Trouillier $\mathrm{HH}$, Hausdorf J, von Schulze Pellengahr C. Medial branch blocks versus pericapsular blocks in selecting patients for percutaneous cryodenervation of lumbar facet joints. Reg Anesth Pain Med 2007; 32:27-33.

206. Pham Dang C, Lelong A, Guilley J, Nguyen JM, Volteau C, Venet G, Perrier C, Lejus C, Blanloeil Y. Effect on neurostimulation of injectates used for perineural space expansion before placement of a stimulating catheter: Normal saline versus dextrose $5 \%$ in water. Reg Anesth Pain Med 2009; 34:398-403.

207. Indahl A, Kaigle AM, Reikeräs O, Holm $\mathrm{SH}$. Interaction between the porcine lumbar intervertebral disc, zygapophysial joints, and paraspinal muscles. Spine (Phila Pa 1976) 1997; 22:2834-2840.

208. Indahl A, Kaigle A, Reikerås $\mathrm{O}$, Holm S. Electromyographic response of the porcine multifidus musculature after nerve stimulation. Spine (Phila Pa 1976) 1995; 20:2652-2658.

209. Johnson CR, Barr RC, Klein SM. A computer model of electrical stimulation of peripheral nerves in regional anesthesia. Anesthesiology 2007; 106:323-330.

210 Eippert F, Finsterbusch J, Bingel U, Büchel C. Direct evidence for spinal cord involvement in placebo analgesia. Science 2009; 326:404.

211. Tsui BC, Kropelin B, Ganapathy S, Finucane B. Dextrose $5 \%$ in water: Fluid medium maintaining electrical stimulation of peripheral nerve during stimulating catheter placement. Acta Anaesthesiol Scand 2005; 49:1562-1565.

212. Holm I, Friis A, Brox JI, Gunderson R, Steen $H$. Minimal influence of facet joint anesthesia on isokinetic muscle performance in patients with chronic degenerative low back disorders. Spine (Phila Pa 1976) 2000; 25:2091-2094.

213. Kang YM, Choi WS, Pickar JG. Electrophysiologic evidence for an intersegmental reflex pathway between lumbar paraspinal tissues. Spine (Phila Pa 1976) 2002; 27:E56-E63.

214. Bhatia MT, Parikh LCJ. Epidural saline therapy in lumbo-sciatic syndrome. J Indian Med Assoc 1966; 47:537-542.

215. Gupta AK, Mital VK, Azmi RU. Observations of the management of lumbosciatic syndromes (sciatica) by epidural saline. J Indian Med Assoc 1970; 54:194-196.

216. Wittenberg RH, Greskötter KR, Steffen $R$, Schoenfeld BL. Is epidural injection treatment with hypertonic saline solution in intervertebral disk displacement useful? (The effect of $\mathrm{NaCl}$ solution on intervertebral disk tissue). Z Orthop Ihre Grenzgeb 1990; 128:223-226.

217. Levin JH. Prospective, double-blind, randomized placebo-controlled trials in interventional spine: What the highest quality literature tells us. Spine J 2009; 9:690-703.

218. O'Neill C. Re: Manchikanti L, Singh V, Falco FJE, et al. Cervical medial branch blocks for chronic cervical facet joint pain. A randomized, double-blind, controlled trial with one-year follow-up. Spine 2008, 33:1813-20. Spine (Phila Pa 1976) 2009; 34:1117-1118.

219. Louhiala P, Puustinen R. Rethinking the placebo effect. J Med Ethics 2008; 34:107-109.

220. Louhiala P. The ethics of the placebo in clinical practice revisited. J Med Ethics 2009; 35:407-409.

221. Concato J. When to randomize, or "evidence-based medicine needs medicinebased evidence." Pharmacoepidemiol Drug Saf 2012; 21:6-12.

222. Quill TE, Holloway RG. Evidence, preferences, - recommendations - finding the right balance in patient care. $N$ Engl J Med 2012; 366:1653-1655.

223. Gelijns AC, Gabriel SE. Looking beyond translation - integrating clinical research with medical practice. $N$ Engl J Med 2012; 366:1659-1661.

224. Blease C. The principle of parity: The "placebo effect" and physician communication. J Med Ethics 2012; 38:199-203.

225. Manchikanti L, Benyamin RM. Key safety considerations when administering epidural steroid injections. Pain Manag 2015; Jun 10:1-12. [Epub ahead of print]

226. Manchikanti L, Staats PS. Nampia- 
parampil DE, Hirsch JA. What is the role of epidural injections in the treatment of lumbar discogenic pain: A systematic review of comparative analysis with fusion and disc arthroplasty. Korean J Pain 2015; 28:75-87.

227. Manchikanti L, Falco FJE. Safeguards to prevent neurologic complications after epidural steroid injections: Analysis of evidence and lack of applicability of controversial policies. Pain Physician 2015; 18:E129-E138.

228. Manchikanti L, Hirsch JA. Neurological complications associated with epidural steroid injections. Curr Pain Headache Rep 2015; 19:482.

229. Manchikanti L, Pampati V, Benyamin RM, Boswell MV. Analysis of efficacy differences between caudal and lumbar interlaminar epidural injections in chronic lumbar axial discogenic pain: Local anesthetic alone vs. local combined with steroids. Int J Med Sci 2015; 12:214-222.

230. Manchikanti L, Singh V, Pampati V, Falco FJE, Hirsch JA. Comparison of the efficacy of caudal, interlaminar, and transforaminal epidural injections in managing lumbar disc herniation: Is one method superior to the other? Korean J Pain 2015; 28:11-21.

231. Manchikanti L, Falco FJE, Pampati V, Hirsch JA. Lumbar interlaminar epidural injections are superior to caudal epidural injections in managing lumbar central spinal stenosis. Pain Physician 2014; 17:E691-E702.

232. Manchikanti L, Falco FJE, Benyamin RM, Gharibo CG, Candido KD, Hirsch JA. Epidural steroid injections safety recommendations by the Multi-Society Pain Workgroup (MPW): More regulations without evidence or clarification. Pain Physician 2014; 17:E575-E588.

233. Manchikanti L, Candido KD, Singh V, Gharibo CG, Boswell MV, Benyamin RM, Falco FJE, Grider JS, Diwan $S$, Hirsch JA. Epidural steroid warning controversy still dogging FDA. Pain Phy- sician 2014; 17:E451-E474.

234. Manchikanti L, Candido KD, Kaye AD, Boswell MV, Benyamin RM, Falco FJE, Gharibo CG, Hirsch JA. Randomized trial of epidural injections for spinal stenosis published in the New England Journal of Medicine: Further confusion without clarification. Pain Physician 2014; 17:E475-E488.

235. King W, Lau P, Lees R, Bogduk N. The validity of manual examination in assessing patients with neck pain. Spine J 2007; 7:22-26.

236. Carragee EJ, Haldeman S, Hurwtiz E. The pyrite standard: The Midas touch in the diagnosis of axial pain syndromes. Spine J 2007; 7:27-31.

237. Bogduk N. In defense of King et al: The validity of manual examination in assessing patients with neck pain. Spine J 2007; 7:749-752.

238. Carragee EJ, Haldeman S, Hurwtiz E. Response to Bogduk. Letter to the Editor. Spine J 2007; 7:752-753.

239. Bogduk N, McGuirk B. Causes and sources of chronic low back pain. In: Bogduk N, McGuirk B (eds). Medical Management of Acute and Chronic Low Back Pain. An Evidence-Based Approach: Pain Research and Clinical Management. Volume 13. Elsevier Science BV, Amsterdam, 2002, pp 115-126.

240. Barnsley L, Lord SM, Wallis BJ, Bogduk $\mathrm{N}$. The prevalence of chronic cervical zygapophyseal joint pain after whiplash. Spine (Phila Pa 1976) 1995; 20:20-26.

241. Lord SM, Barnsley L, Wallis BJ, Bogduk N. Chronic cervical zygapophysial joint pain with whiplash: A placebo-controlled prevalence study. Spine (Phila Pa 1976) 1996; 21:1737-1745.

242. Manchikanti L, Boswell MV, Singh V, Pampati V, Damron KS, Beyer CD. Prevalence of facet joint pain in chronic spinal pain of cervical, thoracic, and lumbar regions. BMC Musculoskelet Disord 2004; 5:15.
243. Manchikanti L, Pampati V, Damron KS, McManus CD, Jackson SD, Barnhill RC, Martin JC. A randomized, prospective, double-blind, placebo-controlled evaluation of the effect of sedation on diagnostic validity of cervical facet joint pain. Pain Physician 2004; 7:301-309.

244. Manchikanti L, Damron KS, Rivera J, McManus CD, Jackson SD, Barnhill RC, Martin JC. Evaluation of effect of sedation as a confounding factor in the diagnostic validity of lumbar facet joint pain: A prospective, randomized, doubleblind, placebo-controlled evaluation. Pain Physician 2004; 7:411-417.

245. Barnsley L, Lord S, Wallis B, Bogduk $\mathrm{N}$. False-positive rates of cervical zygapophysial joint blocks. Clin J Pain 1993; 9:124-130.

246. Manchikanti L, Cash KA, Pampati V, Fellows B. Influence of psychological variables on the diagnosis of facet joint involvement in chronic spinal pain. Pain Physician 2008; 11:145-160.

247. Manchikanti L, Boswell MV, Manchukonda R, Cash KA, Giordano J. Influence of prior opioid exposure on diagnostic facet joint nerve blocks. J Opioid Manage 2008; 4:351-360.

248. Pampati S, Cash KA, Manchikanti L. Accuracy of diagnostic lumbar facet joint nerve blocks: A 2-year follow-up of 152 patients diagnosed with controlled diagnostic blocks. Pain Physician 2009; 12:855-866.

249. Manchikanti L, Pampati S, Cash KA. Making sense of the accuracy of diagnostic lumbar facet joint nerve blocks: An assessment of implications of $50 \%$ relief, $80 \%$ relief, single block or controlled diagnostic blocks. Pain Physician 2010; 13:133-143.

250. Saal JS. General principles of diagnostic testing as related to painful lumbar spine disorders. A critical appraisal of current diagnostic techniques. Spine (Phila Pa 1976) 2002; 27:2538-2545. 